Portland State University

PDXScholar

Summer 6-29-2017

\title{
Symbiosis with Nitrogen-fixing Rhizobia Influences Plant Defense Strategy and Plant-predator Interactions
}

Adrienne Louise Godschalx

Portland State University

Follow this and additional works at: https://pdxscholar.library.pdx.edu/open_access_etds

Part of the Biology Commons, and the Plant Sciences Commons Let us know how access to this document benefits you.

\section{Recommended Citation}

Godschalx, Adrienne Louise, "Symbiosis with Nitrogen-fixing Rhizobia Influences Plant Defense Strategy and Plant-predator Interactions" (2017). Dissertations and Theses. Paper 3644.

https://doi.org/10.15760/etd.5528

This Dissertation is brought to you for free and open access. It has been accepted for inclusion in Dissertations and Theses by an authorized administrator of PDXScholar. Please contact us if we can make this document more accessible: pdxscholar@pdx.edu. 
Symbiosis with Nitrogen-fixing Rhizobia Influences Plant Defense Strategy

and Plant-predator Interactions

by

Adrienne Louise Godschalx

A dissertation submitted in partial fulfillment of the requirements for the degree of

Doctor of Philosophy

in

Biology

Dissertation Committee:

Daniel J. Ballhorn, Chair

Todd N. Rosenstiel

Sarah M. Eppley

Jason Podrabsky

Yangdong Pan

Portland State University

2017 
(C) 2017 Adrienne Louise Godschalx 


\begin{abstract}
As sessile organisms, plants evolved a plethora of defenses against their attackers. Given the role of plants as a primary food source for many organisms, plant defense has important implications for community ecology. Surprisingly, despite the potential to alter entire food webs and communities, the factors determining plant investment in defense are not well-understood, and are even less understood considering the numerous symbiotic interactions in the same plant. Legume-rhizobia symbioses engineer ecosystems by fixing nitrogen from the atmosphere in trade for plant photosynthates, yet connecting symbiotic resource exchange to food web interactions has yet to be established. Here I test how rhizobia influence plant defense and tritrophic interactions in lima bean (Fabaceae - Phaseolus lunatus L.): a model plant in chemical ecology research characterized by a broad range of different defenses. Examining suites of traits among lima bean genotypes, highly cyanogenic cultivars and wild type plants (high cyanotypes) produce more hook-shaped trichomes, as a putative combined approach of chemical and mechanical defenses, forming defense syndromes to protect against multiple feeding guilds (Chapter 2). Testing costs that may have contributed to forming tradeoffs among strategies, high cyanotypes show reduced fitness under plant-plant competition relative to low cyanotypes, but when challenged with herbivory, high cyanotypes fitness
\end{abstract}


reductions are no longer evident (Chapter 3). Young leaves, not reproductive organs, are the most cyanogenic lima bean organ, and removal quantitatively decreases fitness, supporting assumptions that the most valuable tissues will be most highly defended (Chapter 4). Testing the degree to which nitrogenfixing rhizobia contribute to cyanogenesis, high cyanotypes form more nodules than low cyanotypes. Quantitative relationships between nodule number and plant traits highlight the role symbiotic investment plays a role in plant defense and nutritive phenotype, while simultaneously, genotypically-determined levels of defense shape plant investment in symbiosis (Chapter 5). Interestingly, traits that trade off by cyanotype (i.e. high cyanogenesis but low indirect defense) reflect the patterns in plants with nitrogen-fixing rhizobia. Rhizobia-inoculated lima beans show reduced indirect defenses, recruiting fewer parasitoid wasps (Chapter 6) and predatory ants (Chapter 7). Examining plant-ant attraction in greater detail, ants prefer headspace regions above EFN droplets, corresponding with species-specific differences in suites of volatiles, indicating EFN, like floral nectar, can be scented to manipulate insect behavior (Chapter 8). Overall, understanding when investing in traits to recruit predators is more effective than investing in defensive chemistry, and how particular ecological contexts, such as symbioses can influence the outcome of defense allocation strategies remains a fascinating area of research. Determining the mechanisms 
underlying why rhizobia and other belowground microbial symbionts influence their host plants' aboveground interactions, whether plants traits affected by symbiotic microbes are simply a function of the costs and benefits from resource exchange, or whether symbionts can influence the success of primarily direct versus indirectly defended plants is an important question for understanding complex trophic systems and connecting to agricultural implications for more effective biological pest control. 


\section{Dedication}

For Roots and Canopy 


\section{Acknowledgements}

The work presented in my dissertation is truly the product of the support from many wonderful mentors, collaborators, research assistants, peers, and friends. I am grateful for the time and energy that went into editing my writing, counting nodules, keeping my research plants alive, and listening to my ideas, even the less-than-reasonable ones with a mentoring kindness that made me believe I could make it all happen.

I am incredibly grateful to my $\mathrm{PhD}$ advisor, Dr. Daniel Ballhorn, and my dissertation committee, Dr. Sarah Eppley, Dr. Todd Rosenstiel, Dr. Jason Podrabsky, and Dr. Yangdong Pan. I recently heard a presenter state, "You are the average of the five people with whom you choose to surround yourself." If this applies to the five people listed above that I chose to serve on my committee, my average would be significantly more talented, patient, empowering, and curious-qualities of my academic mentors that have gotten me to where I am today with the opportunity to write an acknowledgements section. Specifically, I am grateful for Sarah's approach to ecology and timely perspectives that inspired me many times. Todd's never-ending challenge to seek a bigger question empowered me to create work of which I am proud. Jason responded with alacrity and caring support in the face of most challenges I brought to him-including joining my committee midway. Dr. 
Pan never failed to respond to my stream of statistical questions with a lighthearted patience. Thank you all. It is an honor to have you on my team.

In deep appreciation for my committee chair, Dr. Daniel Ballhorn's guidance has been an integral part of my development as a scientist, poising me to contribute to the global scientific community. For that, I could not be more grateful. Dan supported my wild ideas, yet also provided helpful feedback and wisdom at important moments. It has been a privilege to work with Dan for the past five years.

Our community in Ballhorn Lab has grown throughout these five years as well. Thank you to Dr. Steffi Kautz, for training and guidance invaluable to me throughout my graduate career. To Brett Younginger, Emily Wolfe, Mehmet Balkan, and Nathan Stewart for being an incredible team and significantly affecting my appreciation for fungi.

My ever-evolving team of research assistants that volunteered their time to run chemical assays, count trichomes, water plants, write drafts, and keep me company, never failed to brighten my day. A special thank you to all students that worked with me on these projects: postbacs- Tanya Smagula, Katie Baxter, Aramee Diethelm, Julie Trisel, and Trevor Williams; undergraduates (PSU)- JG Bradner, Fidel Gonzales-Ferrer, Vy Tran, Katya Davidson, Alf Fink, Omar Cazares, Barbara Olvera, Ben Watzig, Brady Hall, 
Brit George, Luke Weinstein; (PCC)- Bodhi Zamudio, Whitney Korenek; (Queens University)- Lynne Stracovsky; high school students- Vy Nguyen, Lisa Grimm, and Katie Woodhouse. Each of you have made my $\mathrm{PhD}$ a better body of work and my time at Portland State far more fun.

I want to specifically thank the unsung heroes, my unofficial support team, that serve many students as they pass through Portland State. Linda Taylor, Greenhouse Manager, relentlessly kept my plants' best interests in mind, keeping plants alive and healthy enough for experimental integrity. Wentai Luo, Senior Research Associate, graciously trained me to run GCTOF-MS samples and carefully analyze my data. Marc Nisenfeld, Miracle Worker for the Science Support Shop, translated many of my crazy ideas into laser-cut and gas-tight reality. Leah Tuor, Ian Garrett, Judy Ngai, Lindsay Henderson, and Megan Simon, guardians of the Biology Department, for the paper trails and emails that make all of this work possible.

Learning how to communicate and connect with varying audiences about my work has been a journey that I have not taken alone. Thank you to Cat Dayger for serving as my first line of peer-review in our symbiotic editing arrangement. Thank you to the long list of mentors that periodically agreed to meet with me over coffee, OMSI Science Communication Staff, Office of Graduate Studies 3MT staff, and the Sigma Xi Columbia-Willamette Board of Directors for helping me learn how to tell a more powerful story. Thank you vii 
to my non-science friends, family, and guests at the Andina bartop for any time you did not understand my work, challenging me to communicate what I am trying to say more clearly and in a more compelling, personal manner.

To all my family and friends, thank you for being my constant cheerleader, enduring my existential crises, and listening over and over to my latest lima bean stories. Mom, you are a courageous beacon of kindness and I can only hope to learn to wield the warmth of your love to connect with people the way that you do. Daddio, your passion and eye for beauty in the world are incredible gifts. Seeing the world in this light, I fear I will never be able to stop seeking ways to illuminate the beauty of the natural world.

On a personal note, I want to thank my Biology Department community of faculty, grad students, et. al for creating an atmosphere that lifts each other up, embraces diverse fields of study, and is constantly hungry for greater impact and seminar cookies. We have a wonderful community here at Portland State Biology. Create the community you love, love the community you create.

The work I present here was funded by Portland State University's Forbes-Lea and David T. Clark awards and the National Science Foundation through the Graduate Research Fellowship Program (GRFP), Doctoral 
Dissertation Improvement Grant (DDIG), and other funding awarded to Dr. Daniel Ballhorn (award IDs: 2014159631, 1457369, and 1501420).

As a final word, thank you again Dan. I look forward to the Google Scholar alerts filled with your future discoveries and the conference poster sessions, where we will meet to scheme up new questions as colleagues. 


\section{Table of Contents}

Abstract.............................................................................

Dedication....................................................................

Acknowledgements.........................................................

List of Tables...............................................................

List of Figures.............................................................

Chapter 1

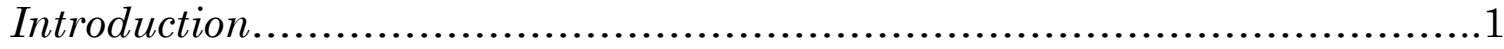

Chapter 2

Lima bean defense syndromes..............................................10

Chapter 3

Cyanogenesis in the absence of herbivory reduces fitness

Chapter 4

Which plant organs are most important to defend?

Chapter 5

Cyanotypes differ in rhizobia colonization

Chapter 6

Plants with rhizobia attract fewer parasitoid wasps....

Chapter 7

Plants with rhizobia attract fewer ants.

Chapter 8

Ants distinguish between extrafloral nectar scents

Chapter 9

Conclusions....

Literature Cited. 


\section{List of Tables}

Table 2.1

Quantitative variability of defensive traits in lima bean (Phaseolus

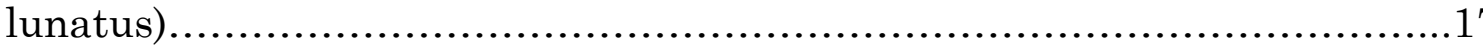

Table 3.1

Results of the nested ANOVAs on the effects of competition and type of competition (interspecific, inter-cyanotypic, intra-cyanotypic) on vegetative and generative traits of $\mathrm{HC}$ - and LC lima bean plants.........................50

Table 3.2

Results of the nested ANOVAs on the effects of competition and type of competition (interspecific, inter-cyanotypic, intra-cyanotypic) on vegetative and generative traits of HC- and LC lima bean plants...........................51

Table 8.1

Species differences in EFN volatiles vary by chemical class...................161 


\section{List of Figures}

Figure 2.1

AFLP-based dendrogram of lima bean genotypes...............................20

Figure 3.1

Effects of different competition treatments and cyanotype of lima bean (mean

$\pm \mathrm{SE})$

Figure 3.2

Log-response ratios for vegetative and generative traits of HC- and LC-plants for the influence of a interspecific competition by corn, b inter-cyanotypic competition, and c intra-cyanotypic competition (means $\pm \mathrm{CI}$ )..................53

Figure 3.3

Effects of inter-cyanotypic competition, cyanotype, and herbivory on vegetative traits of lima beans (mean $\pm \mathrm{SE}$ )

Figure 3.4

Effects of inter-cyanotypic competition, cyanotype and herbivory on generative traits of lima beans (mean $\pm \mathrm{SE}$ )

Figure 3.5

Effects of inter- cyanotypic competition and herbivory on generative traits of the HC-genotype of lima beans (mean $\pm \mathrm{SE}$ ) ..................................55

Figure 3.6

Comparison of predicted (calculated from separate responses to competition and herbivory) and observed responses to the simultaneous effect of herbivory and competition for HC- and LC-plants of lima bean (mean \pm CI)

Figure 4.1

Cyanogenic potential ( $\mathrm{HCNp}$; a) and soluble protein content (b) of different lima bean organs.................................................................... 79

Figure 4.2

Tissue consumed by generalist herbivores....

Figure 4.3

Pod and seed production following simulated florivory or folivory

Figure 4.4

Simulated folivory treatment experimental design 
Figure 5.1

Nodulation and leaf traits differences between cyanotypes across time

Figure 5.2

Quantitative relationships between nodule number and variation in plant traits.

Figure 6.1

Parasitoid wasps preferred rhizobia-free plants over rhizobia-inoculated plants among natural community

Figure 6.2

Nodule number quantitative relationships with leaf damage and parasitoid

visitation

Figure 6.3

Plant below- and aboveground biomass did not vary by rhizobia treatment.

Figure 6.4

Plant above- and belowground biomass quantitative relationships to parasitoid recruitment.

Figure 7.1

Ant recruitment onto Phaseolus lunatus plants with and without

rhizobia 139

Figure 7.2

Final reduced structural equation model for the relationship between rhizobia, aboveground P. lunatus plant traits (including cyanogenic potential, HCNp, and plant nutritive quality, expressed as total soluble protein), and ant abundance.............................................................. 140

Figure 7.3

$P$. lunatus plant traits with and without rhizobia for young, intermediate,

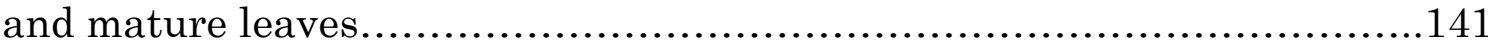

Figure 8.1

Ant bioassay design to test ant attraction based on volatile headspace of nectar droplets without contact. 
Figure 8.2

Ants distinguish EFN from controls without contact..........................166

Figure 8.3

Ants choose host plant EFN based on scent...................................167

Figure 8.4

Suites of EFN volatiles vary by plant species.................................168 


\section{Chapter 1: Introduction}

Plant biochemistry fundamentally influences the function of all terrestrial ecosystems on our planet. Photosynthesis splits water in the presence of light in order to form energy-rich organic bonds from atmospheric carbon dioxide. Carbon assimilation plays a foundational role in all food webs, making plants the target for a wide diversity of mammals, insects, fungi, and other primary consumers. Despite being rooted in place, plants are hardly defenseless as they respond to a vast diversity of herbivore attackers with an array of biochemical processes, resulting in compounds that are either directly toxic to herbivores (Zenk, 1967; Seigler \& Price, 1976) or recruit predators for protection (Turlings et al., 1990). Both direct and indirect defenses influence herbivore behavior (Hermann \& Thaler, 2014), nutrition (Ballhorn et al., 2007; Veyrat et al., 2016), and survival (Tigreros et al., 2017). Therefore, the food web, from producer to consumer depends on plant chemical biology.

Over time, herbivores attack, plants defend, herbivores develop resistance, and plants defend with greater intensity or novel strategies in a dynamic coevolutionary arms race between plants and consumers (Ehrlich \& Raven, 1964). Increasing resource allocation to defense may lead to greater reproductive success in the face of strong herbivore pressure, but such investment can also reduce plant fitness due to the resource and energetic 
costs of defense to the plant (Ballhorn et al., 2014a). Different defense traits require varying amounts of resources such as precursor molecules, complex enzymatic pathways, ATP and reducing power-all of which may conflict with plant growth and primary metabolism (Nunes-Nesi et al., 2010). High levels of defense are less beneficial in the absence of herbivore attack, but invaluable when effective against the correct enemy.

Herbivore-specific defense strategies have led to a staggering diversity of chemicals. Plants produce chemical defenses that are either toxic in small concentrations (qualitative defenses), such as cyanogenic glucosides (Poulton, 1990), glucosinolates (Brown et al., 1991), and alkaloids (Nuringtyas et al., 2014), or cumulatively increase in toxicity or inhibit digestion when ingested in greater quantities (quantitative defenses), such as phenolic compounds (Faeth, 1985), or various peptides including protease inhibitors (Broadway \& Duffey, 1986). Plants reinforce chemical defenses by producing varying degrees of mechanical defenses such as trichomes, spines, prickles, and thickened leaf cuticles. Some plants tolerate high degrees of defoliation with only minor fitness consequences, or invest in rapid growth patterns to spatially "escape" attack, minimizing the need to invest in synthesizing costly defense compounds (McNaughton, 1983). Many defenses are not constitutively produced, but are inducible in response to herbivore-associated cues, which are often transmitted via volatile organic compounds (VOCs). 
In addition to direct chemical and physical defenses, plants can deploy volatile chemicals advertise localized herbivore attack to other organisms that benefit from finding the same herbivores. Natural enemy recruitment, or indirect defenses, can effectively protect plant tissues from damage by attracting predators that kill or aggressively evict herbivores from the plant surface. Plants draw the attention of the third trophic level through specific VOCs (Turlings et al., 1990) and rewards such as sugar-rich extrafloral nectar (EFN) (McLain, 1983).

Herbivore feeding behavior is simultaneously shaped by chemical defenses, mechanical defenses, and host plant quality (i.e. bottom up effects) as well as top-down pressure from predators (Read et al., 2009; Moles et al., 2013). Combinations of interacting plant traits emerge that most effectively reduce fitness costs from herbivore attack, resulting in plant defense syndromes, or suites of traits (Agrawal \& Fishbein, 2006). Direct interference between compounds or hormonal regulation can drive trait tradeoffs in addition to resource allocation shifts (Ballhorn et al>, 2010b; Wei>et al>, 2014a). Observing which traits appear in the same plants at the same time can reveal information about each trait's role in the greater ecological backdrop shaping plant defense expression. Describing patterns and underlying processes that explain the context-dependency of plant defense interactions has been an interesting challenge in chemical ecology. Lima 
bean (Phaseolus lunatus, Fabaceae) is a model system in chemical ecology with well-characterized suites of defenses (Frehner \& Conn, 1987; Kost \& Heil, 2005; Ballhorn et al., 2005, 2008a; Ballhorn, 2011b)

Lima bean cultivars and wild-type genotypes express a gradient of cyanogenic potential, the capacity to release toxic hydrogen cyanide from preformed precursors (Nienhuis et al., 1995). Using these consistent and welldocumented cyanogenic-specific genotypes (cyanotypes) with either high cyanogenesis, $\mathrm{HC}$, or low cyanogenesis, LC, the relationship can be examined between cyanogenesis as a direct chemical defense and the expression of other defense traits in lima bean. High cyanogenic potential interferes with anti-pathogenic polyphenol oxidases (Ballhorn et al., 2010b), and HC plants produce relatively lower amounts of indirect defense traits (Ballhorn et al., 2008b). Low cyanotypes show the opposite pattern, being more effectively protected against fungal pathogens, and more proficient at recruiting predators than their highly cyanogenic relatives. Tradeoffs between cyanogenesis and various other defenses compromise one mode of defense in favor of another. All plants employ a combination of several protection mechanisms, and examining the intersectionality of such defense syndromes reveals covarying strategies that may have a synergistic influence on fitness (Agrawal \& Fishbein, 2006). To test the role of mechanical defense in the lima bean defense syndromes with either high or low cyanogenic potential, I 
quantify hook-shaped trichomes in various $\mathrm{HC}$ and LC genotypes in Chapter 2. Building a more comprehensive picture of plant defenses that includes mechanical defenses contributes to understanding how both cyanotypes functionally defend against multiple feeding guilds.

Whether a defensive trait increases or decreases plant fitness depends entirely on the plants' ecological context. Plant competition could pose a challenge to plants investing heavily in defensive enzymes and precursors, as is the case with cyanogenesis (Frehner \& Conn, 1987). In Chapter 3, I test the fitness costs of producing high or low levels of cyanogenic potential when plants are faced with competition across a gradient of intensity, with and without herbivore pressure. Leading hypotheses aim to describe expression patterns based on each defensive trait's and trait combination's fitness costs and advantages (Stamp, 2003).

Fitness costs and benefits associated with any particular defensive trait or combinations of traits can be challenging to quantify, yet are essential in developing plant defense theory. In Chapter 4, I test one component of the Optimal Defense Hypothesis (ODH) (McKey, 1974). Withinplant allocation, where within the plant defenses are allocated, assumes that plant organs most critical for fitness will be most highly defended, leading us to test the cyanogenic potential of various lima bean organs, including leaves, 
flowers, and pods (Godschalx et al., 2016). By creating a spatial map of within-plant allocation, and subsequently testing the fitness effects of removing varying percentages of those organs, a central tenet of this hypothesis can be tested to gain insight into how herbivore damage in nature impacts on plant survival.

Identifying the optimal timing and allocation for growth and defense in response to a diversity of interspecific interactions is a dynamic process for plants, both individually and over evolutionary time. Soil microbial symbionts add to the incredible complexity of interspecific interactions that influence plant resource allocation by priming induced defenses, (Van der Ent et al., 2009; Pineda et al., 2012), acting as pathogens \#\#, or provisioning raw materials to the plant. Plant- Nitrogen-fixing bacteria, such as rhizobia that associate with legume roots in the family Fabaceae, provide access to plantavailable nitrogen assimilated from atmospheric dinitrogen. Direct access to this otherwise limited nutrient implies a reduction in the costs of protein-rich biological processes and nitrogen-demanding defenses. In lima bean, rhizobia positively increase both growth and cyanogenic potential (Thamer et al., 2011). In Chapter 5, I tested the relationship between quantitative defense level and plant symbiotic association by counting nodules and measuring the corresponding chemical phenotypes. I wanted to understand whether nitrogen obtained from greater numbers of root nodules would directly 
influence quantitative defense expression, or if genotypically-determined defense levels could force plants to maintain greater colonization to meet cyanotype-specific nitrogen demands. Costs of a larger carbon sink resulting from maintaining symbionts for more nitrogen-fixation in root nodules may have previously unmeasured impacts on the food web (Pringle, 2015a).

Predators and parasitoids respond to specific plant chemistry, and are sensitive to changes in plant volatile signals and extrafloral nectar (EFN): sugar-rich nectar reward not associated with pollination. Both plant traits require significant unrecoverable quantities of assimilated carbon. Rhizobia also have heavy carbon requirements; ca. $16-30 \%$ is directed to root nodules (Peoples et al., 1986; Kaschuk et al., 2009). The strong carbon sink associated with root nodules may explain why lima bean VOCs decreased overall and shifted in composition in plants with rhizobia (Ballhorn et al., 2013c). The influence of rhizobia on indirect defense traits and the predator/parasitoids' response to rhizobia-mediated plant chemistry from the bottom up is not well-characterized (Rasmann et al., 2017). To understand whether rhizobia alter investments in VOCs and natural enemy attraction, I quantify recruitment of parasitoid wasps in natural communities in Costa Rica in Chapter 6, ultimately aiming to understand whether rhizobia have food web impacts that extend to the third trophic level, in this case, to parasitoid wasps. 
Ants are another predator with a widespread and intimate evolutionary history with plants. Harnessing the aggressive power of ant colonies can be an effective shield against herbivores. Ant-plants range from obligatory myrmecophytes, complete with a unique set of symbiont-specific traits, to facultative myrmecophiles, most of which rely on secreting EFN. Most plants that secrete EFN do so via the facultative model, with EFN production being induced in response to signals of herbivore pressure in order to conserve carbohydrates until needed. Given the carbon-rich nature of this trait, I tested whether rhizobia, which act as a carbon sink, impose a constraint on EFN secretion in Chapter 7. Using a structural equation model, I tested the influence of rhizobia on several plant traits, including soluble protein, cyanogenic potential, below- and aboveground biomass, and EFN to assess which traits mediated by rhizobia could affect the recruitment of ant patrollers.

Facultative EFN as a reward for predators only functions as a plant defensive trait if the predators are able to find this resource and respond with aggressive behavior towards plant antagonists at the appropriate times. Despite being an extensively-studied interaction, volatile components in EFN that could serve as a recruiting signal have not been examined (\#\#). In Chapter 8, I sampled the volatile profiles of several distantly-related species' EFN, and compare ant attraction. The potential for EFN to be scented, and 
the potential mechanism it may serve in ant attraction, provides plants with a mechanism to communicate with and manipulate insect symbionts.

Plants evolved their diverse suite of defensive traits in the context of interacting enemies and mutualists, above and belowground. By employing natural enemies, and maintaining nitrogen-fixing root nodules, plants factor cooperation into growth and defense allocation dynamics. Cooperative relationships and resource costs benefitting another species present an interesting challenge to evolutionary biology. To explain how mutualistic relationships persist, hypotheses based on host sanctioning and partner choice have been extensively tested in both ant-plant and legume-rhizobia relationships. Examining how forces that facilitate persistence of cooperation through evolutionary time will contribute to our understanding of plantherbivore interactions and food web energy flow. 


\section{Chapter 2: Lima bean defense syndromes}

Published as: Ballhorn DJ, Godschalx AL, Kautz S. 2013. Co-variation of chemical and mechanical defenses in lima bean (Phaseolus lunatus L.). Journal of Chemical Ecology 39: 413-417.

Abstract Plants usually express multiple chemical and mechanical defenses simultaneously. The interplay of these defenses is still poorly understood, as predictions range from negative associations such as allocation tradeoffs to positive correlations forming synergistic defense syndromes. Surprisingly, little empirical evidence exists on the co-variation of multiple plant defenses. In the present study, we analyzed different genotypes of lima bean (Phaseolus lunatus L.) for the expression of two direct chemical defenses [cyanogenic potential (constitutive), polyphenol oxidase activity (inducible)], two indirect chemical defenses [volatiles (VOCs) and extrafloral nectar (EFN; both inducible)] and a constitutive mechanical defense (hook-shaped trichomes). While the occurrence of trichomes was positively correlated with cyanogenesis, these traits showed a tradeoff with polyphenol oxidase activity, release of VOCs, and secretion of EFN. Hook-shaped trichomes were abundantly present in four of 14 genotypes investigated, and were found only in one monophyletic group of an AFLP-based tree, thus indicating a single evolutionary origin within the species. Our findings show that different lima bean genotypes express either one of two defense systems: 1) high 
constitutive defense via cyanogenesis and trichomes or 2) high inducible defense via VOCs, EFN, and PPO activity.

Introduction: In accordance with their manifold attackers, plants usually express multiple defenses simultaneously, including chemical and mechanical defenses. While some defenses show tradeoffs due to resource allocation constraints, others are positively correlated forming defense syndromes (Agrawal, 2011). Lima bean (Phaseolus lunatus) is a model plant in chemical ecology for which various defenses have been well studied (Kost \& Heil, 2006; Ballhorn, 2011b). Cyanogenesis, the wound-induced release of hydrogen cyanide (HCN) from cyanogenic glucosides (CNGs) represents a constitutive direct chemical defense against many herbivores. When plant tissue is damaged, preformed vacuolar CNGs react with apoplastic Bglucosidases releasing toxic HCN. Polyphenol oxidases (PPOs) catalyze the oxygen-dependent oxidation of phenols to reactive quinones and represent an inducible direct defense — mostly against pathogens (Ballhorn, 2011b). In addition to these direct chemical defenses, indirect defenses of lima bean have been particularly well studied. Herbivore-induced volatile organic compounds (VOCs) serve as an indirect defense against herbivores by chemically signaling to parasitoids; inducible secretion of extrafloral nectar (EFN) is highly efficient in attracting predatory ants (Kost \& Heil, 2006). In our previous studies, we showed distinct tradeoffs between cyanogenesis and 
VOCs as well as cyanogenesis and PPO-activity (Ballhorn et al., 2008b;

Ballhorn, 2011b). The interaction of cyanogenesis and PPO-activity is unique in that there is direct interference of these traits as cyanide released from damaged cells inhibits PPO activity (Ballhorn et al., 2010b). In contrast to the well characterized chemical defenses of lima bean, their co-variation with mechanical defenses has not been studied. Here, for the first time, we report the occurrence of defense-associated hook-shaped trichomes, analyze the covariation of this mechanical defense with chemical defenses, and discuss the expression of these defenses in a phylogenetic context. Our study provides insight into the evolution of different defense syndromes within a single plant species.

Methods and Materials: Lima bean plants were grown from seeds provided by the Leibniz Institute of Plant Genetics and Crop Plant Research (IPK; Gatersleben, Germany), the Max Planck Institute for Chemical Ecology (Jena, Germany), as well as from seeds collected from natural populations in Colombia and Mexico (see Table 2.1 for details). Plants were cultivated under greenhouse conditions according to Ballhorn (2011). All plant individuals (N=10 per genotype) were analyzed for all traits considered in this study. The cyanogenic potential (HCNp; concentration of cyanogenic precursors) was quantified by using the Spectroquant ${ }^{\circledR}$ cyanide test (Merck) (Ballhorn et al., 2005). Enzymatic activity of PPOs was measured by quantification of the $\mathrm{O}_{2}$ - 
consumption during oxidation of polyphenols and their derivates to quinones (Ballhorn et al., 2010b). Extracts were prepared from leaves inoculated with the generalist fungal plant pathogen Colletotrichum gloeosporioides by application of spore solutions adjusted to 105 spores ml-1 according to Ballhorn et al. (2010). Extraction was carried out in $1.5 \mathrm{ml}$ Eppendorf ${ }^{\circledR}$ tubes at $25{ }^{\circ} \mathrm{C}$ using Sörensen buffer allowing for enzymatic decomposition of cyanogenic precursors in the plant extracts. For further details, see Ballhorn et al. (2010). Trichomes were quantified microscopically (Leica MZ16) by counting their number along a $0.5 \mathrm{~cm}$ segment of a first-order leaf vein of mature leaves. One segment per leaf was evaluated $(\mathrm{N}=10$ leaves per genotype, each derived from a different plant individual). VOCs released from plants induced by spraying jasmonic acid (JA; $1 \mathrm{mmolL}^{-1}$ aqueous solution) were collected over $24 \mathrm{~h}$ on charcoal filters in a closed loop stripping set-up (circular airflow in a closed headspace) and were qualitatively and quantitatively analyzed on a GC-Trace mass spectrometer (Trace GC Ultra DSQ; Thermo Electron, Austin, TX, USA). For detailed information on the program used for separation and for information on compound identification and quantification, see Ballhorn et al. (2008). EFN production was quantified according to Kost and Heil (2006). After spraying plants (24 h) with JA and subsequent mechanical damage of leaves ( 15 holes per $\mathrm{cm}^{2}$ leaf blade, diameter of individual holes $0.2 \mathrm{~mm}$ ), EFN production was quantified as 
amounts of soluble solids by using microcapillaries (PCR Micropipets 1-10 $\mu$; Drummond) for determination of volume and a brix refractometer for determination of sugar concentration. We generated a dendrogram of all 14 lima bean genotypes by using AFLPs, and we mapped the level of each defense on the tree. The dataset was based on a subset of samples that had previously been published, and methods are described in detail in Ballhorn et al. (2008).

Results and Discussion: Lima bean genotypes included here showed different but distinct combinations of defensive traits. Five genotypes were high cyanogenic (HC) (HCNp between 49 and $101 \mu$ mol HCN g ${ }^{-1} \mathrm{fw}$ ), whereas nine showed significantly lower HCNp (Table 2.1). HCNp of these lowcyanogenic (LC) plants was between 3 and $31 \mu \mathrm{mol} \mathrm{HCN} \mathrm{g-1} \mathrm{fw.} \mathrm{In} \mathrm{line} \mathrm{with}$ our previous studies, HC-plants released quantitatively less volatiles upon JA-induction (Table 2.1). These two defenses were significantly negatively correlated (Pearson's correlation: $\mathrm{r}=-0.787, p<0.001, N=40$ ). Furthermore, HCNp and EFN ( $\mathrm{r}=-0.843, p<0.001)$ as well as HCNp and activity of PPOs $(\mathrm{r}=-0.909, p<0.001)$ were negatively correlated. While cyanogenesis showed a tradeoff with all other chemical defenses considered, this trait was significantly positively correlated to density of defense-associated trichomes $(\mathrm{r}=0.796, p<0.001)$. Four out of five HC-genotypes showed significantly higher numbers of trichomes than LC-genotypes (Table 2.1). Only WT_2233 had 
significantly lower numbers of trichomes than other HC-genotypes. However, regarding all other chemical traits analyzed (PPO-activity, EFN, and VOCs), this genotype showed the typical defense system of HC-plants (Figure 2.1). The combination of high chemical defense through cyanogenesis and mechanical defense by trichomes was found exclusively in one monophyletic group of an AFLP-based tree indicating a single evolutionary origin of trichomes within the species (Figure 2.1). Thus, despite variation in the combination of individual traits, lima bean expresses either one of two defense systems: 1) High levels of cyanogenesis, a direct chemical defense against herbivores, usually coupled with mechanical anti- herbivore defense via trichomes; or 2) high levels of VOCs and EFN, as indirect defenses against herbivores, coupled with high levels of PPOs, enzymes mainly involved in resistance to pathogens (Figure 2.1). Defense system 1) is constitutive, whereas defense system 2) is composed of inducible traits. One genotype (WT_2233) was high cyanogenic and was nested within a low cyanogenic clade and had no trichomes. Otherwise, high cyanogenic plants with trichomes occurred only in an exclusive clade; genetic conservation of this trait combination seems likely. However, the basis of the observed tradeoffs and positive correlations that we report here remains unknown. Constraints in resource allocation or evolutionary adaptations to suites of attackers dominating under distinct environmental conditions are potentially 
underlying causes of such contrasting defense systems. In the case of $\mathrm{HCNp}$ and PPO-activity, negative associations between plant defenses on the physiological level have been demonstrated (Lieberei et al., 1989; Ballhorn et al., 2010b; Ballhorn, 2011b). Free cyanide released upon cell damage quantitatively inhibits PPOs but also many other metal-containing enzymes involved in plant defense against herbivores and pathogens such as catalases and peroxidases (Kranthi et al., 2003). Thus, in the light of multiple inhibiting effects of cyanogenesis on other plant defenses, the combination of this trait with trichomes, which do not interfere with chemical defenses or synthesis pathways, represents a possibility for HC-lima beans to rely on an efficient second type of defense besides cyanogenesis. In particular, this combination of high $\mathrm{HCNp}$ and trichomes may provide simultaneous resistance to chewing herbivores, which cause the release of significant amounts of cyanide, and sucking herbivores, which cause minimal tissue damage and thus limited release of $\mathrm{HCN}$, but are susceptible to trapping by hook-shaped trichomes (Figure 2.1). In support of this hypothesis, these trichomes are found mostly along the leaf veins on the lower surface of leaves, representing the favored feeding location for many sucking insects. Future studies are needed to understand how these different defense systems function in nature when plants are under simultaneous attack by multiple pathogens and insect herbivores. Furthermore, analyzing variation of defense 
systems on spatial scales and identifying correlations to specific habitat

characteristics in combination with transplanting experiments would yield a deeper understanding of the function of these defense systems. Nevertheless, despite further questions, our study shows for the first time, even at the intraspecific level, complex and fundamentally different defense systems occur.

Table 2.1 Quantitative variability of defensive traits in lima bean (Phaseolus lunatus). Values shown for plant traits are means $( \pm \mathrm{SD} ; \mathrm{N}=10$ individual plants per genotype). Significant differences between genotypes were calculated by a post hoc test (Tukey's HSD; $\mathrm{P}<0.001$ ) after one-way ANOVA [cyanogenic potential $(\mathrm{HCNp})(\mathrm{F} 13,126=139.01 ; \mathrm{P}<0.001)$, polyphenol oxidase $(\mathrm{PPO})$ activity $(\mathrm{F} 13,126=50.98 ; \mathrm{P}<0.001)$, volatile organic compounds (VOCs) (F13,126=15.83; P<0.001), extrafloral nectar $(\mathrm{EFN})(\mathrm{F} 13,126=34.56$; $\mathrm{P}<0.001)$, and hook-shaped trichomes $(\mathrm{F} 13,126=111.50 ; \mathrm{P}<0.001)]$ and are indicated by different superscript letters. Source of seeds for plant cultivation is indicated by superscript numbers in parentheses: (1) Leibniz Institute of Plant Genetics and Crop Plant Research (IPK; Gatersleben, Germany), (2) Oaxaca, Mexico (15.925532,-97.151151); (3) Colombia (Pacific coast, exact 
location unknown), (4) Yucatan, Mexico (19.832924,- 87.466335), (5) Max Planck Institute for Chemical Ecology (Jena, Germany) 


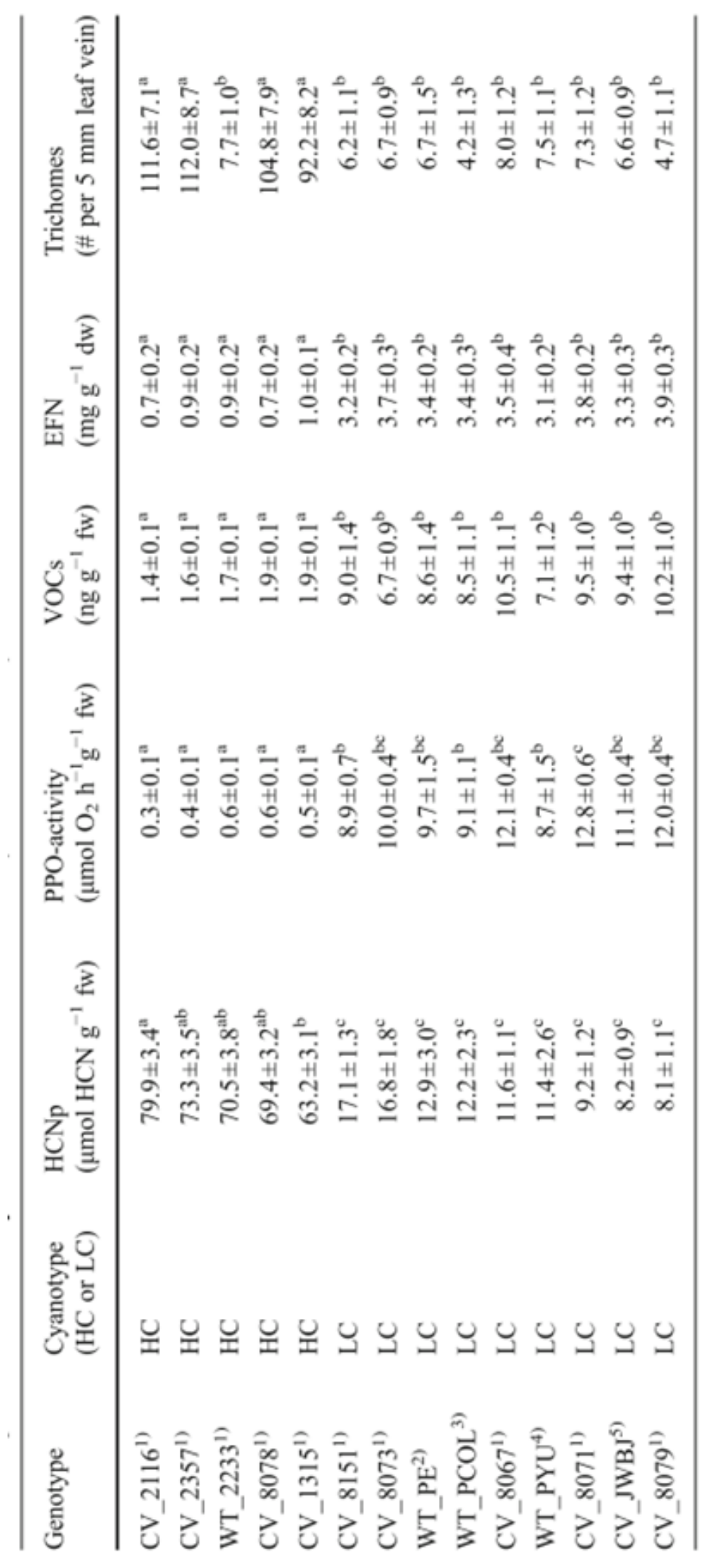


a
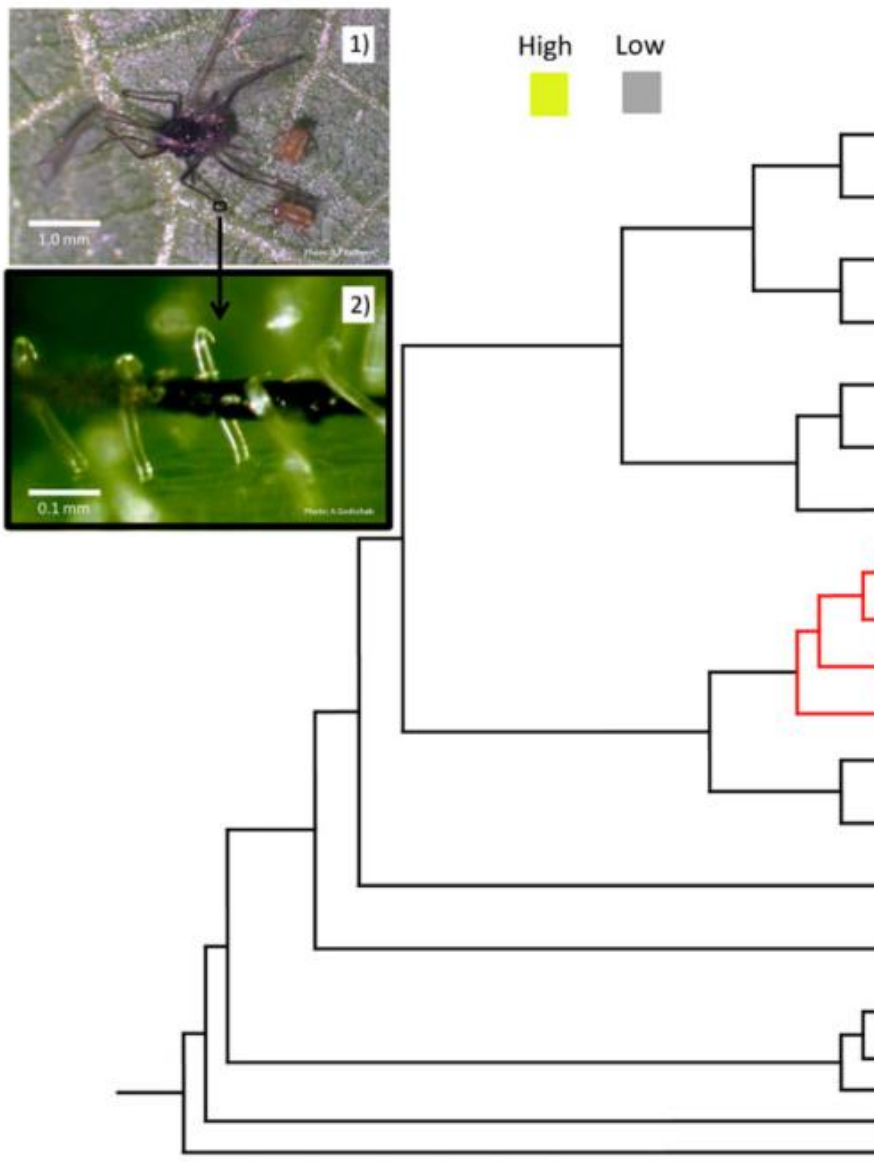

b

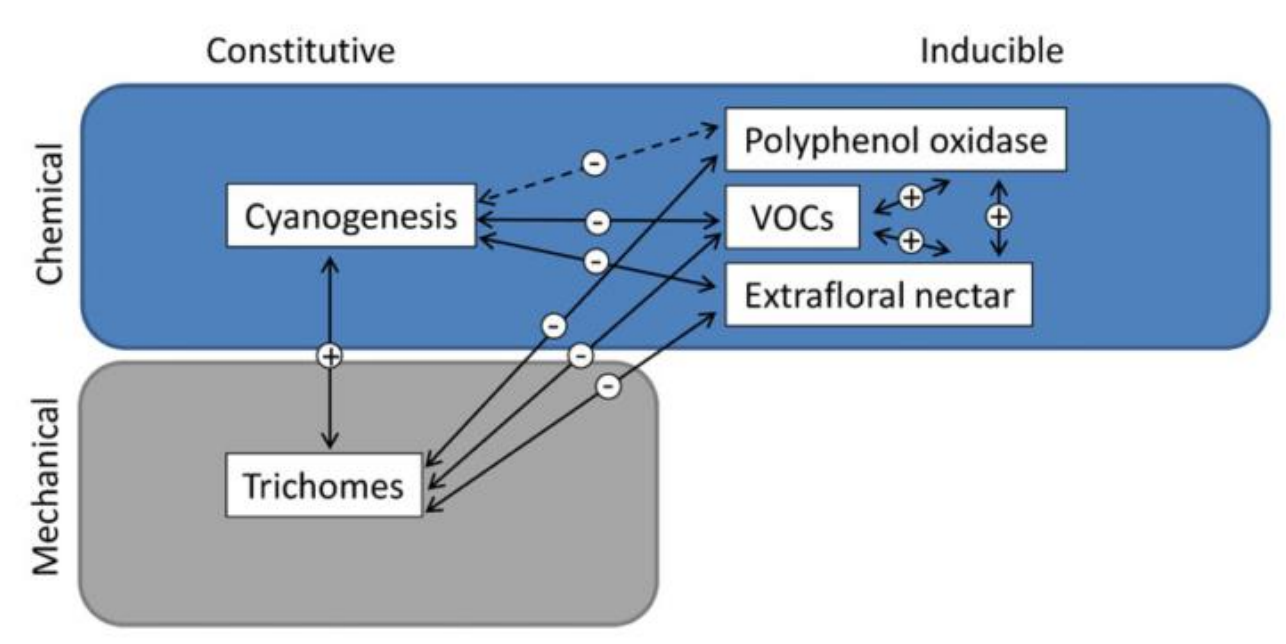

Constitutive Inducible

defenses defenses

High Low

CV_8151A

CV_8151 B

CV $8067 \mathrm{~A}$

CV_8067 B

CV $8073 \mathrm{~A}$

CV_8073 B

CV_8071A

CV_8071 B

WT_PCOLA

WT PCOLB

CV_8079A

CV 8079 B

CV_JWBJ A

CV_JWBJ B

CV 8078 A

C CV_8078 B

CV_1315

CV_2357A

CV_2116

WT $2233 \mathrm{~A}$

WT_2233 B

WT_PYUA

WT_PYUB

WT_PE 26

WT_PE 82

WT_PE 13

WT_PE 14

WT_PE 06

WT PE 08

WT_PE 09

WT_PE 11

WT_PE 12

WTPE 10

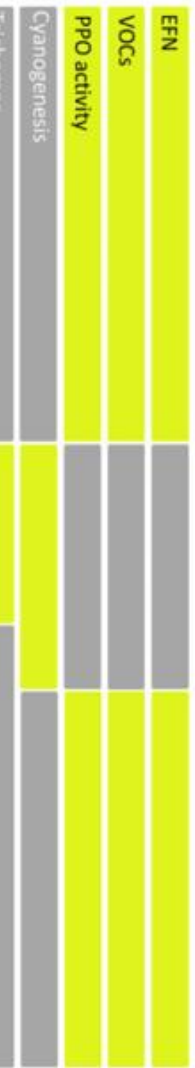


Figure 2.1 AFLP-based dendrogram of lima bean genotypes. A) The tree topology was obtained using PAUP* (Swofford, 2003) after the Nei-Li model (Nei \& Li, 1979) using a subset of samples from Ballhorn et al. (2008). A total of 34 lima bean samples (14 genotypes) with 509 variable bands derived from eight primer combinations were included in the analysis. Phaseolus microcarpus Martius was used as outgroup (not shown). The quantitative expression of trichomes, cyanogenesis, polyphenol oxidase activity (PPOs), herbivore-induced volatile organic compounds (VOCs), and extrafloral nectar (EFN) is indicated with bars. Genotypes that possess hooked-shaped trichomes are highlighted in red and are restricted to one monophyletic group. The dashed line between 'Cyanogenesis' and 'PPOactivity' in B indicates that these traits interact on a physiological level as PPO-activity is inhibited by cyanide. Photo insert 1 shows a female aphid trapped by trichomes on the lower surface of a lima bean leaf (genotype CV_8078). Before its death, the aphid gave birth to two offspring. The upper juvenile is trapped as well. Photo insert 2 shows close-up of an aphid leg trapped by hook-shaped trichomes on the leaf vein. b Co-variation and tradeoffs of various plant defensive traits in lima bean. Cyanogenesis and trichomes are positively correlated, while these two defenses are neg- atively correlated with the inducible defenses PPOs, VOCs and EFN) 


\section{Chapter 3: Cyanogenesis in the absence of herbivory reduces fitness}

Published as: Ballhorn DJ, Godschalx AL, Smart SM, Kautz S, Schädler M. 2014. Chemical defense lowers plant competitiveness. Oecologia 176: 811-824.

Abstract: Both plant competition and plant defense affect biodiversity and food web dynamics and are central themes in ecology research. The evolutionary pressures determining plant allocation toward defense or competition are not well-understood. According to the growth-differentiation balance hypothesis (GDB), the relative importance of herbivory and competition have led to the evolution of plant allocation patterns, with herbivore pressure leading to increased differentiated tissues (defensive traits), and competition pressure leading to resource investment towards cellular division and elongation (growth-related traits). Here we tested the GDB hypothesis by assessing the competitive response of lima bean (Phaseolus lunatus) plants with quantitatively different levels of cyanogenesis—a constitutive direct, nitrogen-based defense against herbivores. We used high (HC) and low cyanogenic (LC) genotypes in different competition treatments (intra-genotypic, inter-genotypic, interspecific), and in the presence or absence of insect herbivores (Mexican bean beetle; Epilachna varivestis) to quantify vegetative and generative plant parameters (above and belowground biomass as well as seed production). 
Highly defended HC-plants had significantly lower aboveground biomass and seed production than LC-plants when grown in the absence of herbivores implying significant intrinsic costs of plant cyanogenesis. However, the reduced performance of HC- compared to LC-plants was mitigated in the presence of herbivores. The two plant genotypes exhibited fundamentally different responses to various stresses (competition, herbivory). Our study supports the GDB hypothesis by demonstrating that competition and herbivory affect different plant genotypes differentially and contributes to understanding the causes of variation in defense within a single plant species.

Introduction: Plants are unable to evade herbivore enemies through movement and show various defensive traits to protect themselves (Atsatt \& O’Dowd, 1976; Heil, 2008; Agrawal et al., 2010). Great variation in plant defensive compounds and strategies of expression has developed over evolutionary time under the selection pressure of diverse herbivore antagonists (Ehrlich \& Raven, 1964; Bernays \& Graham, 1988). Given the importance of plants as primary producers in all terrestrial ecosystems, plant defense has potential to vastly impact entire communities by directly affecting herbivores, and consequentially higher trophic levels (Barton, 2007; Viola et al., 2010). Surprisingly, despite the importance of plant defensive traits for complex food webs, plant defense-associated resource allocation is 
often not well understood. However, an improved understanding of the consequences of plant defense expression is a critical task in ecological research (Herms \& Mattson 1992; Siemens et al. 2002; Stamp 2003; Agrawal 2011; Moles et al. 2013).

Current ecological understanding of plant defense is divided among hypotheses based on resource allocation tradeoffs (Herms \& Mattson, 1992; Agrawal, 2007; Agrawal et al., 2010; Kempel et al., 2011) or positive syndrome-forming correlations (Agrawal \& Fishbein, 2006; Viola et al., 2010; Ballhorn et al., 2013a) such as the optimal defense, carbon-nutrient balance, growth rate, and growth-differentiation balance hypotheses (Stamp, 2003; Ballhorn et al., 2008b; Read et al., 2009; Kaplan et al., 2009; Ballhorn, 2011b; Agrawal, 2011; Nomura et al., 2011; Dantas \& Batalha, 2012; Adler et al., 2012; Moles et al., 2013; Campbell \& Kessler, 2013). Overall trends are difficult to identify as plant defensive strategies vary immensely across and within taxa, involving both chemical and mechanical traits (Moles et al. 2013). Available data are rarely entirely congruent with any one particular defense hypothesis, and often distinct discrepancies between theory and empirical data emerge in plant defense experiments (Barton, 2007; Viola et al. 2010; Agrawal 2011; Thamer et al. 2011). In line with this, the predicted negative correlation between competitive ability and defense was not supported by a recent meta-analysis, that showed a slight positive correlation 
(Viola et al. 2010). In contrast, Kempel et al. (2011) found in a multi-species experiment that the competition-defense trade-off holds for constitutive resistance but is complemented by a positive relationship between competition and induced resistance. The necessity for empirical data on plant resource allocation to defense in different contexts is evident.

Defense compound production is considered costly to the plant, requiring resources which may otherwise be used for growth and reproduction, yet this cost is frequently invisible in empirical data (Siemens et al. 2002). The growth-differentiation balance (GDB) hypothesis uses the difference in resource requirements for cellular growth and photosynthetic net assimilation to explain patterns of plant investment in defense, assuming cellular growth requires more nutrient acquisition than photosynthesis. Defense expression is highest when cellular growth is limited, but photosynthesis is not (Stamp 2003; Barton 2007). The GDB hypothesis provides a theoretical framework in which the cost of defense on a plant's fitness may be assessed, assuming tradeoffs between growth and defense (Herms and Mattson 1992; Kempel et al. 2011; Massad et al. 2012). Often, the high energetic cost of defense is shown as decreased biomass or reproductive performance, demonstrating the plant's lowered fitness, and only few studies show the costs of defense on plant fitness under nutrient or competition stress (Baldwin and Hamilton 2000; Siemens et al. 2002; Marak 
et al. 2003; Barton 2007). An extension of the GDB hypothesis allows insight into the relative importance of herbivores or competitors in limiting plant fitness, resulting in plants with growth or differentiation-dominated life strategies, respectively (Stamp 2003). Often plants form inducible rather than constitutive defenses in order to mitigate high cost of defense compounds (Ballhorn et al., 2008a, 2013a). Thus, plants enhance their level of defense by producing bioactive compounds only when needed, under herbivore attack (Ballhorn et al. 2008a; Kost and Heil 2008; Bennett et al. 2009; Cipollini and Heil 2010). Understanding metabolic costs of defense when plants are exposed to different stressors allows for an integrative approach to testing the GDB hypothesis (Agrawal 2011).

As plants usually are in permanent competition for multiple resources, plant-plant competition likely is a factor critically affecting plant performance in nature (Baldwin and Hamilton 2000; Chase et al. 2002; Siemens et al. 2002; Marak et al. 2003). In addition to competition between different plant species, intra-specific competition strongly affects plant fitness, in particular, as plants of the same species use similar resources and occupy similar spatial dimensions (Broz et al. 2010). Thus, plant genotypes of the same species expressing different defensive traits are ideal experimental systems to disentangle the concerted effects of defense and competition on plant fitness, serving as a control for background genetic variation. Moreover, 
they provide ideal systems to evaluate the importance of genetic diversity within plant species. Different genotypes of lima bean (Phaseolus lunatus L.) investing to different degrees into cyanogenesis (called cyanotypes hereafter), used in our study, represent such an experimental plant system.

Cyanogenesis is the enzymatically accelerated release of toxic hydrogen cyanide (HCN) from cyanide-containing precursors in response to cell damage (Frehner and Conn 1987; Selmar et al. 1989) and provides efficient plant defense against herbivores both in laboratory (Ballhorn \& Lieberei, 2006; Ballhorn et al., 2008b) and field experiments (Ballhorn et al., 2009a, 2013b). Since HCN release is directly correlated with the amount of tissue damaged (Ballhorn et al., 2009a), cyanogenesis is most effective against chewing herbivores (Cork, 1996; Ballhorn et al., 2005). As the cyanide-containing precursors (in lima bean these are the cyanogenic glucosides linamarin and lotaustralin) contain nitrogen, cyanogenesis is considered more costly for a plant to produce and maintain than purely carbon-based defenses (Baldwin et al. 1990; Rask et al. 2000; Ballhorn et al. 2014). Intuitively, producing such a defense would limit the amount of resources available to growth and reproduction. Despite this cost, cyanogenesis occurs in about 10 percent of vascular plant species, and has evolved independently in various families (Frehner and Conn 1987; Jones et al. 1999). The ecological costs of cyanogenesis in the absence of enemies are 
only poorly understood but negative effects on freezing tolerance and plant growth, reproduction, and competitive ability have been postulated (Kakes 1990; Noitsakis and Jacquard 1992; Hayden and Parker 2002).

The current study tests the assumption of the GDB hypothesis that the relative importance of competition and herbivory can shape plant resource allocation patterns. We test this by measuring plant performance under interspecific as well as inter- and intra-genotypic competition and herbivore stress, which has not previously been examined. We hypothesize that low cyanogenic genotypes are less affected by competition when grown under herbivore-free conditions than high cyanogenic genotypes. This may also lead to a competitive advantage over high cyanogenic plants without herbivores. The outcome of the competition between low and high cyanogenic genotypes should depend on the presence of herbivores, which should alleviate the competitive advantage of low cyanogenic genotypes.

Materials and Methods: Plants: In this study we used high (HC) and low (LC) cyanogenic lima bean (Fabaceae: Phaseolus lunatus L.) genotypes. These cyanotypes (HC_2116 and LC_8067) have been used in our previous studies (Ballhorn et al. 2005, 2007, 2008a, 2010) and showed very consistent cyanogenic potential $(\mathrm{HCNp}$, amount of cyanide stored in form of cyanogenic precursors in plant tissues) over multiple generations (HC_2116: 75 $\mu \mathrm{mol}$ 
HCN and LC_8067: 18 $\mu$ mol HCN per gram fresh weight). The genotypes we used are small-seeded landraces from Central and South America and were selected based on their very consistent chemical and morphological features from a larger set of lima bean genotypes which are in permanent culture in our lab. Plants were cultivated in a greenhouse with additional light provided by a combination (1:1) of HQI-BT 400W (Osram) and RNP-T/LR 400W (Radium) lamps with a light regime of 13:11 L:D under photon flux density of 450-500 $\mu \mathrm{mol}$ photons $\mathrm{m}^{-2} \mathrm{~s}^{-1}$ at table height. Temperature was set at $30^{\circ} \mathrm{C}$ during the light period and at $23^{\circ} \mathrm{C}$ during the dark period. Relative air humidity was adjusted to $70-80 \%$. Plants were cultivated in plant-containers of $10 \times 10 \times 11 \mathrm{~cm}$ (width, length, height; one plant per pot) in a 1:1 ratio of potting soil (Fox Farms, Arcata, CA, USA) and sand (grain size 0.5-2.0 mm). Plants were not inoculated with rhizobia (Thamer et al. 2011; Ballhorn et al. 2013c) and none of the experimental plants showed spontaneous nodulation. After development of the primary leaves all plants were fertilized with $80 \mathrm{~mL}$ of a $0.2 \%$ aqueous solution of Flory-3® $[\mathrm{NPK}+\mathrm{Mg}(\%) ; 15,10,15+2$-Fertilizer, EUFLOR GmbH, Munich, Germany] once to provide for an initial nutrient supply sufficient for plant growth. Plants were watered daily with tap water and were cultivated until the end of their reproductive phase (10-12 weeks after germination). 
Insects: Mexican bean beetles (Coccinellidae: Epilachna varivestis) used in experiments on the effect of herbivory on plant-plant competition were reared on non-cyanogenic snap bean (Phaseolus vulgaris) to avoid conditioning of insects to lima bean cyanotype. These insects are oligophagous herbivores with a narrow host range including various legumes but with distinct preference for lima bean (LaPidus et al., 1963; Flanders, 1984; Dover et al., 1988). Both adults and larvae feed on foliage. Mexican bean beetles were maintained in cages $(1.0 \times 1.0 \times 0.8 \mathrm{~m}, \mathrm{l}: \mathrm{h}: \mathrm{w})$ under greenhouse conditions chosen for plant cultivation (see above). Freshly hatched larvae were used for herbivore treatments.

Experimental setup: We applied seven experimental treatments to test for competitive ability of HC- and LC-plants under herbivore free conditions. Competition experiments were designed to display defense costs under increasing competitive intensity. This increasing competitive intensity ranged from no competition (plants grown alone, controls), to interspecific competition, to intra-specific competition with plants of a different or the same genotype. Plants of the same genotype are considered to require more similar resources as compared to plants of another genotype or another species (Broz et al. 2010). Specifically, in two treatments, individual HC- and LC-plants each were cultivated in combination with corn (Poaceae: Zea mays) (HC vs. corn; LC vs. corn). In another two treatments, HC-plants were 
competing against LC-plants (inter-genotypic competition, HC vs. LC), and in a further competition treatment, HC- and LC-plants were competing against plants from the same cyanotype (intra-genotypic competition, HC vs. HC; LC vs. LC). In control (no competition) experiments, single HC- and LC-plants were analyzed for growth and reproduction.

In addition to experiments under herbivore free conditions, in three treatments we tested for the impact of insect herbivory on HC- and LC-plants with and without competition. Larvae of the Mexican bean beetle were added to HC- and LC-plants growing individually in pots (5 larvae per plant). In addition to these non-competition experiments we tested the impact of herbivory on HC- and LC-plants competing against each other (HC vs. LC; 10 larvae per pot, 5 larvae were placed on each plant). Herbivores were added when plants had developed their first secondary (trifoliate) leaf about two weeks after germination and were left on the plants until the end of the experiment. While herbivores showed limited movement during their first 2-3 larval developmental stages, and thus were mostly restricted to the plant they were placed on, larger larvae were able to easily move from one plant to the other. The movement of herbivores between plants was built into the setups to allow the ecologically-relevant response of plant rejection as a food source by this oligophagous insect based on level of plant defense. Plants with insects were covered with gauze in pairs to avoid escaping of herbivores. In 
previous experiments covering plants with gauze $(N=12$ covered and noncovered plants respectively) proved not to significantly affect plant growth ( $t$ test; $P=0.49$ ) due to only slightly reduced light availability. In fact, plant biomass (dry weight) was almost identical for covered and non-covered plants (covered plants: $3.92 \pm 0.38$; non-covered: $3.89 \pm 0.43$; mean \pm SD). Pots were arranged in a block design (6 blocks with 1 pot of each of the 10 specific combinations of plants and herbivores). Position of pots of the different treatments within the blocks was set at random and the position of blocks within the greenhouse was exchanged every 5 days to avoid chamber effects.

Data collection and analyses: Lima bean plants were analyzed for production of above- and belowground biomass, biomass of reproductive structures (pods) as well as for number and weight of produced seeds. Roots of plants were separated from shoots, washed thoroughly until all soil was removed, and both below- and aboveground plant parts were dried in an oven $\left(65^{\circ} \mathrm{C}\right)$ until constancy of weight (5 days). Dry or almost dry pods were collected per plant, dried at room temperature for two weeks, seeds were removed, counted and individually weighed to the nearest 0.001g (Mettler Toledo, New Classic MF MS303SE). The weight of pods was determined separately. Mean seed weight was calculated as total seed weight per plant divided by the number of seeds. 
Since HC- and LC-plants grew together in the same pots in the intercyanotype competition and combined competition and herbivory treatments they cannot be treated as independent replicates in a common analysis. We therefore used half of the pots with LC-plants as focal plants and the other half with HC-plants as focal plants. Further, in the intra-cyanotype competition treatments we a priori defined one plant as target plant and the other plant as competitor. Values of the corresponding competitors were not used for the analyses.

We used the dataset to perform two different analyses. The first analysis focused on the effects of different types of competition on vegetative and generative traits. For this, we used all herbivore-free treatments and a nested ANOVA model with block factor, cyanotype, two levels of competition (without/with), and three types of competition (interspecific, inter-genotypic, intra-genotypic) nested within the factor competition. Significant effects of type of competition were followed by a post-hoc test (Tukey's). To further assess differences in the responses of LC- and HC-plants to the different competition treatments we calculated log-response ratios for the different variables and compared them using paired t-tests. Log-response ratios are a commonly used method to determine and compare symmetrical and independent strengths of experimental factors (Hedges et al. 1999). In this case, log-response ratios (LR) for the different competition treatments were 
calculated for each block as $L R=\log$ (response variable in the competition treatment / response variable without competition). Thus, values above zero would indicate a positive effect of competition whereas values below zero would indicate negative effects of competition on the measured variable. We used the $95 \%$ - confidence interval to test for deviations from zero.

The second analysis included the treatments LC- and HC- plants alone, in competition with the other cyanotype, and with and without herbivores. For this analysis we used log-transformed data since deciding if there is an interaction between competition and herbivory may depend on the scale of data. Both herbivory and competition affect per-gram loss or growth rates, and their joint effects should be therefore multiplicative on a linear scale even if they do not interact and work independently. On a logarithmic scale, however, a non-significant interaction would in fact indicate an additive response. Thus, a biologically meaningful interaction between herbivory and competition is therefore not necessarily indicated by statistically significant interactions on a linear scale (Rees and Brown 1992; Schädler et al. 2007). We therefore analyzed log-transformed data by means of a four-factorial ANOVA with block, cyanotype, herbivory, and (intercyanotypic) competition. Only ANOVAs with a significant interaction between cyanotype, herbivory and/or competition were followed by a post-hoccomparison (Tukey's test) of means. Since most LC-plants did not reproduce 
with herbivory, mean pod weight and mean seed weight have been excluded from the analysis due to the very low number of valid observations.

Interacting effects of competition and herbivory were analyzed in more detail using response indices (Haag et al. 2004; Schädler et al. 2007). For this, we calculated herbivory response $(H R)$ as a measure of the separate effect of herbivory in the absence of competition as $H R=$ response variable (with herbivores)/response variable (without herbivores) and competition response $(C R)$ as a measure of the separate effect of competition in the absence of herbivory as $C R=$ response variable (with competition)/response variable (without competition) within each block. The predicted total response $\left(T R_{\text {pred }}\right)$ of plants to the combined effects of herbivory and competition in the absence of any interacting effects would be $T R_{\text {pred }}=H R \times C R$. The observed total response $\left(T R_{\text {true }}\right.$ ) was calculated as $T R_{\text {true }}=$ response variable (with herbivores and competition)/response variable (without herbivores and competition). Again, the response indices were log-transformed and the resulting logresponse ratios were analyzed by means of a two-way ANOVA with block and the "method of calculation" (predicted vs. true) as factors. Interactions between herbivory and competition are indicated by a significant value for the "method of calculation" factor. Some of the LC-plants did not produce any pods in the herbivory treatments, resulting in zero-values of the response variable (number of pods, number of seeds) which causes log-ratios to be 
undefined. However, eliminating zero values would mean to ignore potentially meaningful responses. Thus, we added the lowest value of the response variable to all values of these variables, which gives a conservative estimate of the corresponding log-response ratio (see Viola et al. 2010).

However, again mean pod weight and mean seed weight have been excluded from the analysis in this case due to the very low number of valid observations.

\section{Results:}

Type of competition- When grown without competition, LC-plants produced more shoot biomass, more pods, more seeds, and more seeds per pod than HC-plants, whereas mean seed mass was lower for these plants (paired ttests, all $P<0.05)$. Remarkably, the number of seeds was about four times higher for LC-plants than for HC-plants. The total seed mass per plant was higher for LC-plants (mean \pm standard deviation: $5.65 \pm 3.30$ ) than for HCplants $(1.85 \pm 1.13, P<0.001)$ indicating that the lower seed mass does not outweigh the higher seed production. Root biomass was not affected by cyanotype (paired t-test, $P>0.05$ ). Both HC- and LC-plants showed consistently lower shoot- and root biomass under competition than without competition (Table 1, Fig. 1A, B). The extent of this effect did not depend on the type of competition. The negative effect of competition on root biomass 
was stronger for HC-plants (significant competition x cyanotype interaction, Table 1).

The number of pods per plant was generally lower under competition and this effect was further mediated by the cyanotype and the type of competition (significant interaction, Table 1, Fig. 1C). Interspecific competition had a less strong negative effect than inter-cyanotypic competition only for LC-plants. The number of seeds and mean pod weight followed this pattern, i.e., a stronger reduction due to inter-cyanotype competition than due to interspecific competition was only observed for LC plants (Fig. 1D, E). The number of seeds per pod and mean seed weight were not affected by the competition treatments.

Log-response ratios also revealed that most vegetative and generative traits were negatively affected by the three types of competition (Fig. 2A-C). In accordance with the analysis above, only for LC plants, inter-cyanotypic competition caused stronger reductions in seed and pod number than interspecific competition. Differences between the responses of cyanotype were more obvious under interspecific competition with corn, resulting in often weaker effects on LC-plants. More specifically, there was no significant response of root biomass of LC-plants grown in competition with corn (Fig. 2A) and the same cyanotype (Fig. 2C). Mean seed mass generally did not respond to intra-cyanotypic competition for either cyanotype and to inter- 
cyanotypic competiton in HC-plants. The number of seeds per pod was only (positively) affected for HC-plants grown in intra-genotypic competition (Fig. 2C). Apart from one exception (number of seeds per pod for intra-cyanotypic competition), responses of both cyanotypes were only significantly different in the (interspecific) competition treatment with corn. Here, shoot biomass, the number of pods and mean pod mass were less strongly negatively affected in LC-plants whereas mean seed mass was more strongly negatively affected in LC-plants and the number of seeds per pod more strongly positively affected for HC-plants.

Shoot biomass of corn was not affected by the cyanotype of lima bean (LC-plants: $6.60 \pm 0.64, \mathrm{HC}$-plants: $6.44 \pm 0.64$, means \pm standard error; ANOVA: $\left.F_{1,5}=0.03, P=0.87\right)$. Root biomass of corn was considerably higher in competition with lima bean but this was only marginally significant for plants grown in competition with LC-plants (LC-plants: $1.49 \pm 0.21$, HCplants: $0.85 \pm 0.21$, means \pm standard error; ANOVA: $F_{1,5}=4.55, P=0.086$ ).

\section{Interacting effects of herbivory and competition}

Vegetative traits were affected by interactive effects of between cyanotype, competition and herbivory (significant three-way interaction, see Table 2 , Fig. 3A, B). While LC-plants consistently showed a strong decrease of shoot and root biomass due to competition and herbivory, the responses of HC- 
plants to herbivory were weak without competition, or even positive under competition with LC-plants. As a result, HC-plants with herbivory did not suffer from competition in terms of above- and belowground biomass. The interactive effect of herbivory and competition is consistent across cyanotypes but the negative effects of herbivory were not substantially stronger under competition (i.e., there is a significant competition $\mathrm{x}$ herbivory interaction, but there is no three-way interaction competition $\mathrm{x}$ herbivory $\mathrm{x}$ cyanotype, Table 2, Fig. 4A, D). The number of pods and the number of seeds were strongly reduced by herbivory in LC-plants, whereas in HC-plants, herbivory only had a negative effect on the number of pods (significant herbivory $\mathrm{x}$ cyanotype interaction, Table 2, Fig. 4D, E). Further, competition decreased the number of pods in both cyanotypes, but this effect was more pronounced for LC-plants (significant competition x cyanotype interaction, Table 2, Fig. 4C). In HC-plants, the number of seeds per pod was only affected by herbivory (Table 3, without herbivory: $0.71 \pm 0.07$, with herbivory $1.83 \pm 0.07$; means \pm standard error). Pod mass of HC-plants was higher for plants grown alone without herbivores than in all other treatments and seed mass of HCplants was lower in plants grown with herbivores in competition than in all other treatments (significant herbivory x competition interaction, Table 3 , Fig. 5A, B). 
For HC-plants, the predicted response to the combined effects of herbivory and competition (with the other cyanotype) was negative, and significantly stronger than the observed response for shoot weight (Fig. 6A, ANOVA, method of calculation: $\left.F_{1,5}=194.6, P<0.001\right)$, root biomass $\left(F_{1,5}=\right.$ $18.8, P=0.007)$, number of pods $\left(F_{1,5}=17.4, P=0.009\right)$, number of seeds $\left(F_{1,5}\right.$ $=9.97, P=0.03)$ and mean pod weight $\left(F_{1,5}=18.9, P=0.007\right)$. In contrast, for LC-plants we found no difference between observed and predicted values for shoot weight and root weight (Fig. 6B). Number of pods and number of seeds, however, showed the same pattern as for HC-plants.

Discussion: Few empirical studies highlight the competitive advantage of expensive defense traits in the presence of herbivore pressure and overall consensus among studies combining effects of defense and competitive ability is yet to be achieved (Viola et al. 2010; Moles et al. 2013). The data presented here are evidence of a negative correlation between a constitutive defense and competitive ability, supporting the predictions of the GDB hypothesis.

Individual plant growth strategies- In our study comparing the performance of differently defended plant genotypes under competition and herbivory, we considered a range of generative and vegetative plant traits either directly determining plant fitness (e.g. number of seeds produced per plant; weight of seeds) (Rees, 1995; Fenner \& Thompson, 2005) or serving as indirect 
measures of plant fitness such as biomass (Caswell, 1989; García \& Ehrlén, 2002). When grown without competition, LC-plants developed more shoot biomass, pods, seeds, number of seeds per pod as well as a greater mean pod weight when compared to HC-plants grown alone, indicating intrinsic costs of cyanogenesis. Cyanogenesis is a nitrogen-based plant defense, and as nitrogen often is limited, therefore considered costly for the plant (Kakes, 1990). The nitrogen allocated to the synthesis of cyanogenic glucosides is not available for other nitrogen sinks associated with growth and reproduction. The cyanogenic glycosides linamarin and lotaustralin present in lima bean are derived from the proteinogenic amino acids L-valine and L-isoleucine, thus competing with protein biosynthesis (Ballhorn et al. 2005; Ballhorn 2011). Beyond costs of the cyanogenic glycosides themselves, proteins involved in synthesis, cleavage and transportation of cyanogenic glucosides also require nitrogen, further increasing the cost of plant cyanogenesis. However, while most plant performance metrics noted in this study were lower in HC- compared to LC-plants, belowground biomass production was not significantly different between HC- and LC-lima bean plants. The additional necessity to acquire nitrogen to produce nitrogen-rich defenses may explain why HC-plants invest relatively more in root than in shoot production. Altogether, in treatments in which plants were grown alone, plants showed the highest values for biomass and reproductive structure 
production observed for both HC- and LC-plants, as opposed to treatments with competition or herbivore pressure. This indicates considerable impacts of both herbivory and plant-plant competition on growth and ultimately plant fitness, which is in accordance with the GDB hypothesis (Stamp 2003). Previous studies on the defense system in Plantago also support the GDB hypothesis by demonstrating: i) a fitness cost to producing iridoid glycosides, ii) correlations in defense expression and leaf morphology, and iii) increased expression with plant ontogeny as opposed to decreasing with age, as supported by the optimal defense hypothesis (Marak et al. 2000, 2003; Barton 2007).

In their natural environment lima bean plants and legumes in general are less dependent on soil nitrogen than other plants due to their association with nitrogen-fixing rhizobia (Kempel et al., 2009a). However, as the maintenance of rhizobia requires extensive resource allocation towards the bacterial symbionts, obtaining nitrogen is associated with costs, even for legumes (Simms \& Taylor, 2002). Plants in this study were not inoculated with rhizobia and remained rhizobia-free throughout the experiment. These costs of nitrogen-based defense through cyanogenesis were also found in other systems such as white clover (Trifolium repens) in which LC-plants showed increased biomass compared to HC-plants (Kempel et al., 2011). 
Does cyanogenesis affect competitive ability? In terms of shoot biomass, the effect of competition did not differ between lima bean cyanotypes. In contrast, HC-plants showed a stronger reduction of root biomass under competition than LC-plants. This finding may indicate the intensity of belowground competition, as HC-plants allocated similar amounts of biomass to roots as LC-plants, but produced far less shoot tissue. On the other hand, a high impact of competition on belowground biomass has also been reported for other plant systems. Grasses in a field study had reduced belowground performance under competitive pressure in both nitrogen-rich and nitrogenpoor soils (Wilson \& Tilman, 1991; Casper \& Jackson, 1997). In our system, the nitrogen demands for high cyanogenic plants likely increased the strength of belowground competition. Lowering the overall requirement for nitrogen may have given LC-lima bean plants a competitive advantage, especially when faced with intense belowground competition. Importantly, in our system we show that competition in general, as well as different levels of competition, affected several (mainly generative) traits of high and low cyanogenic plants differently. Thus, not only plant species but also plant genotype may strongly impact the outcome of plant-plant competition (Noitsakis and Jacquard 1992; Johnson 2008). In the lima bean system, the tendency for inter-genotypic competition to act as a strong competitive force for the reproduction of LC-plants was observed, while interspecific 
competition with corn had weaker effects. In contrast, HC-plants showed no differentiation between the different levels of competition. In a direct comparison of the two cyanotypes, LC-plants can be seen as the better interspecific competitor in terms of reproduction, at least in an herbivore-free environment. Thus, the prediction that plants may tolerate interspecific competition better than intraspecific competition (Broz et al. 2010) we could only confirm for LC-plants.

The acyanogenic competitor, Zea mays (a plant not producing cyanogenic precursors or other nitrogen-based chemical plant defenses at significant levels) produced a similar amount of shoot tissue under competition with a LC-lima bean plant as when grown under competition with a HC-plant, indicating the cyanogenic potential of its competitor does not affect shoot production. However, in line with the aforementioned reduction of belowground biomass under competition in grasses (Wilson \& Tilman, 1991; Casper \& Jackson, 1997), the root tissue of Z. mays decreased greatly in the presence of a HC-lima bean plant as compared to a LC-lima bean plant. This observation together with the strong effects on some reproductive traits of LC-plants suggest HC-plants are higher nutrient consumers and thus create a higher competitive pressure for neighboring plants. This is paralleled in other plants as cyanogenic morphs of white clover (Trifolium repens) were shown to utilize resources differently from 
acyanogenic morphs (Noitsakis \& Jacquard, 1992). Cyanogenic morphs experienced greater challenges under intra-morphic competition than did acyanogenic morphs (Noitsakis \& Jacquard, 1992). Since Z. mays root production was more intensely impacted by HC-lima bean plants as compared to LC-lima bean plants, the higher nitrogen requirement of the HC-genotypes seemingly limits the root growth of $Z$. mays. Belowground competition for resources may often be a stronger force than aboveground competition, and competition between plants in low nutrient environments was shown to take place mostly belowground, but with additional nitrogen the competitive pressure took place both above- and belowground (Connell, 1983; Wilson \& Tilman, 1991; Casper \& Jackson, 1997).

Do benefits outweigh costs of defense in the presence of herbivores? Expensive defense compound production is found in many different taxa and has evolved multiple times - the ability to defend can clearly lead to an increase in plant fitness (Campbell \& Kessler, 2013). However, in the context of these two ubiquitous phenomena, both competition and herbivory determine the benefits and costs of plant defense. The interacting nature of both phenomena is reflected in the growth of lima bean plants in our experiment. Both shoot and root biomass were strongly affected in LC-plants by herbivory rather independent of the competitive situation of the plant. In contrast, herbivores had only negligible effects on the biomass of HC-plants growing 
alone, while shoot and root biomass clearly increased in the competition treatment in the presence of herbivores. This can be attributed to the selective feeding of herbivores on the LC-plants thereby giving the HC-plants a competitive advantage.

The costs of herbivory and competition were also obvious for the generative growth of both cyanotypes. The number of pods and seeds decreased in response to both pressures, and the stronger response of LCplants reflects the finding that LC-plants are especially sensitive to intercyanotypic competition (but not to competition in general, see above). Also, the negative effect of herbivory was pronounced for both traits in LC-plants, but (and according to the results of vegetative biomass) rather small for $\mathrm{HC}$ plants in terms of number of pods, or even undetectable for the number of seeds. According to the lower number of pods with no change in number of seeds, the number of seeds per pod increased with herbivory in HC-plants. This effect is consistent across the competition treatments and may be interpreted as a kind of compensatory response. We consider the number of seeds as the most relevant indicator for generative fitness (notably in the absence of substantial effects on seed mass), which is therefore substantially affected by herbivory only in LC-plants, whilst HC-plants seemed to be able to compensate the lower number of pods with increased production of seeds within pods. This demonstrates the increase in relative fitness of plants that 
produce costly defensive metabolites under conditions that favor this investment. Herbivores in combined herbivory and competition treatments likely migrated between plants, probably resulting in selective feeding and consequently stronger herbivory on LC-plants, which would likely occur in cooccurring plants in nature. This also reflects situations in natural systems with a considerable variability of cyanogenesis within and between neighboring plant populations (Richards \& Fletcher 2002). The resulting better plant performance of HC-plants demonstrates how investing resources into costly defense compounds can benefit highly defended plants. This benefit can be interpreted as a result of a competitive advantage of better defended plants due to the selective feeding of herbivores on less defended competitors. This suggests that strong herbivory acts as a selection pressure for increased defense production, despite the fitness costs the plant may endure due to lowered competitive ability (Bixenmann et al., 2013).

Our findings suggest that performance of a given plant genotype depends on the ecological context. In our experimental setting, high cyanogenic plants outperformed LC-plants when herbivore pressure was high. However, when herbivore pressure was low, LC-plants had a competitive advantage. Additional factors have to be considered. For example, cyanogenesis has been demonstrated to trade off with inducible indirect plant defenses against herbivores, the emission of herbivore-induced 
plant volatiles (HIPVs) and the secretion of extrafloral nectar (Ballhorn et al., 2008a, 2013a; Kost \& Heil, 2008). Which of these anti-herbivore defense systems provides the highest fitness advantage under which conditions in natural settings remains to be studied (Ballhorn et al., 2009a). Furthermore, in natural systems plants are frequently attacked by herbivores and pathogens simultaneously. Plant defenses efficient against one attacker might have fundamentally different effects on other plant antagonists. In cyanogenic rubber tree as well as in lima bean, high cyanogenic plant individuals are more susceptible to fungal pathogens than their low cyanogenic conspecifics (Lieberei, 1988; Ballhorn et al., 2010b; Ballhorn, 2011b) adding another layer of complexity to the system. Moreover, a number of abiotic factors have been discussed to contribute to the ecological costs of cyanogenesis (see Kakes 1990 and Hayden \& Parker 2002 for review). Variable environmental conditions have led to the evolution of complex and plastic plant defensive systems even within a single plant species.

Conclusions: The low investment in plant defense resulted in the low cyanogenic genotypes being better able to allocate resources to shoot growth and seed production and at the same time, better tolerate interspecific competition, whereas a high investment in defense limits the ability of HClima bean genotypes to do so, but allowed these HC- plants to resist herbivory more effectively. This could imply that intense competition may act as a 
selective force favoring low expression of expensive, constitutive defenses. Since these same plants also express a high level of inducible indirect defenses (Ballhorn et al., 2008a), selection may have acted positively on these alternative defense traits in the face of competition and limited resources. High cyanogenesis, in contrast, might represent a selective advantage when plants are exposed to constantly high herbivore pressure. This divergence of defense strategies—which occurs both in wild and cultivated forms of lima bean (Ballhorn et al., 2013a) —and variable ability to compete for resources highlights the necessity to understand the ecological factors most intensely affecting plant growth and reproduction in natural populations. Several theories have been formulated to comprise current understanding of plant defense, yet a cohesive, community ecology-based approach is still lacking, which is frequently noted by the scientific community given the importance of understanding plant systems in a changing climate (Herms \& Mattson, 1992; Stamp, 2003; Agrawal, 2011; HilleRisLambers et al., 2012). Therefore, generating empirical quantitative data on competitive ability of plants with varying degrees of expensive defense is critical to strengthening current understanding of plant community dynamics, as well as understanding the evolution of plant defenses. 
Table 3.1 Results of the nested ANOVAs on the effects of competition and type of competition (interspecific, inter-cyanotypic, intra-cyanotypic) on vegetative and generative traits of $\mathrm{HC}$ - and LC lima bean plants.

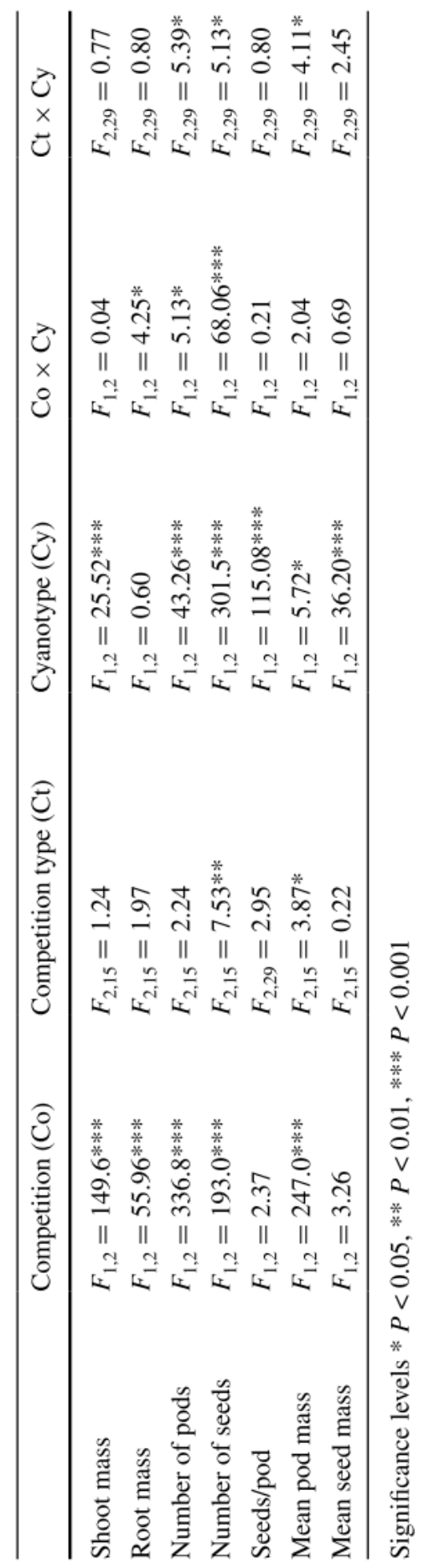


Table 3.2 Results of the nested ANOVAs on the effects of competition and type of competition (interspecific, inter-cyanotypic, intra-cyanotypic) on vegetative and generative traits of $\mathrm{HC}$ - and LC lima bean plants.

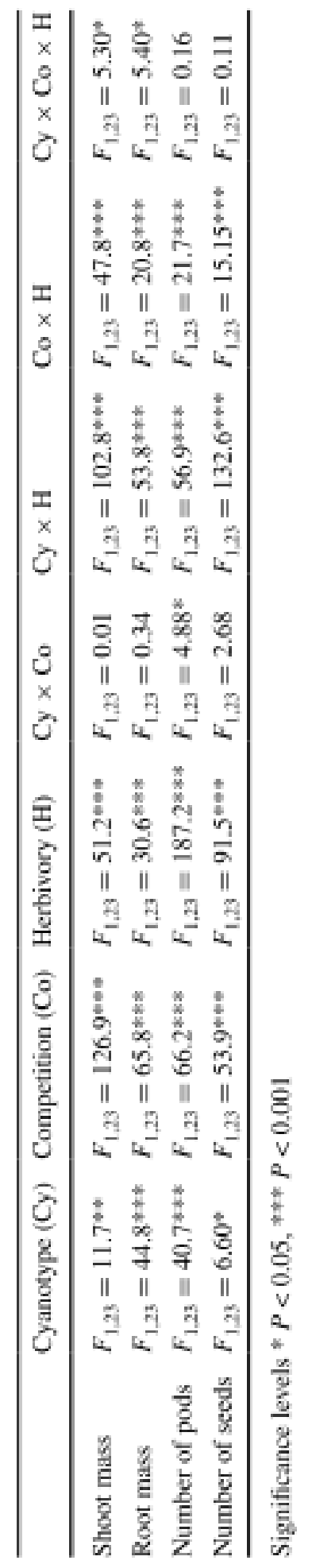



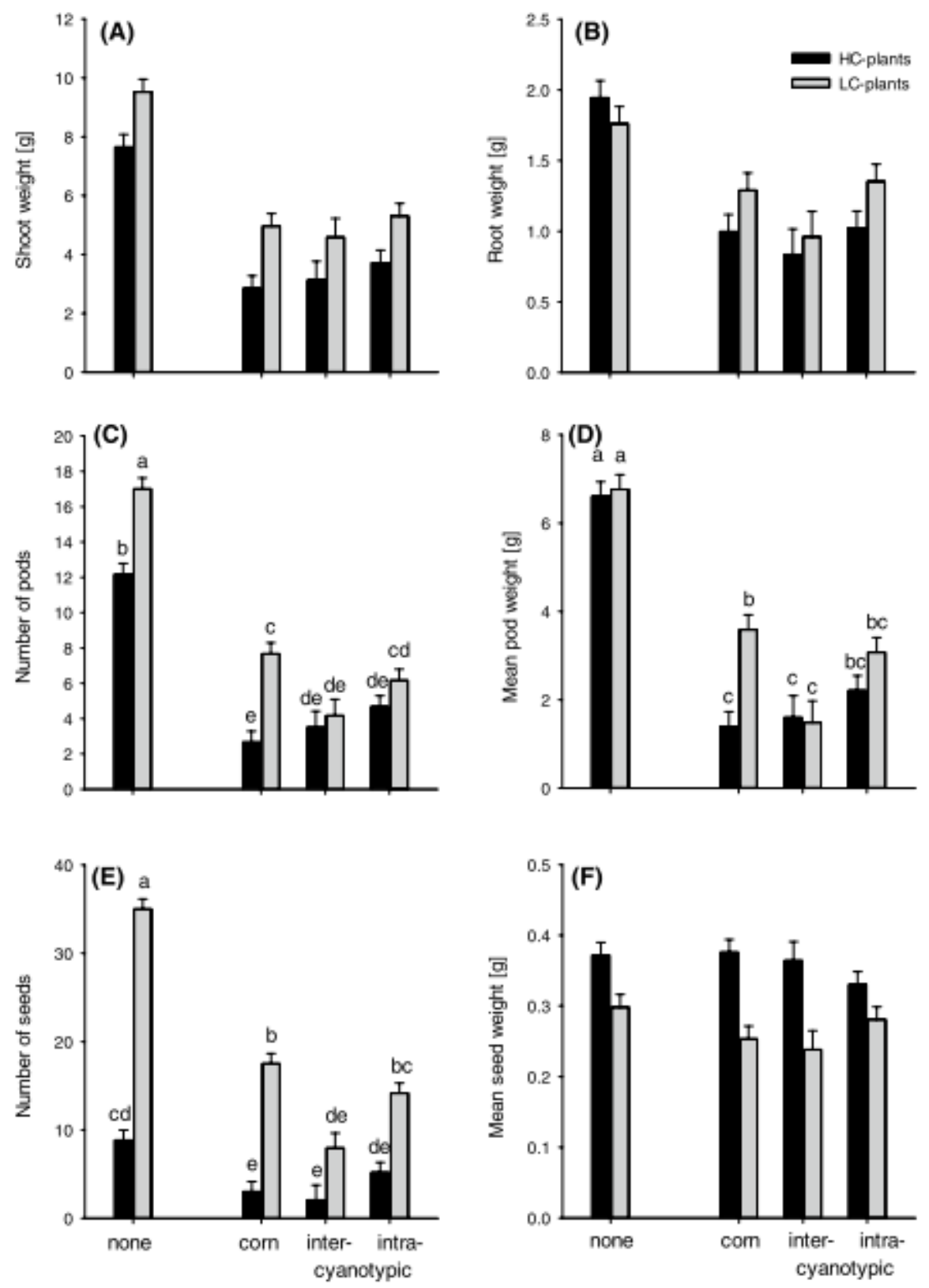

Competition

Figure 3.1 Effects of different competition treatments and cyanotype of lima bean (mean $\pm \mathrm{SE}$ ). None no competition, Corn interspecific competition with corn, Inter-cyanotypic competition with a plant of the other cyanotype, Intra-cyanotypic competition with a plant of the same cyanotype. Different letters indicate significant differences between means following post hoc tests (Tukey's, $\mathrm{P}<0.05$ ). Note that only significant interactions are followed by a post hoc test. 


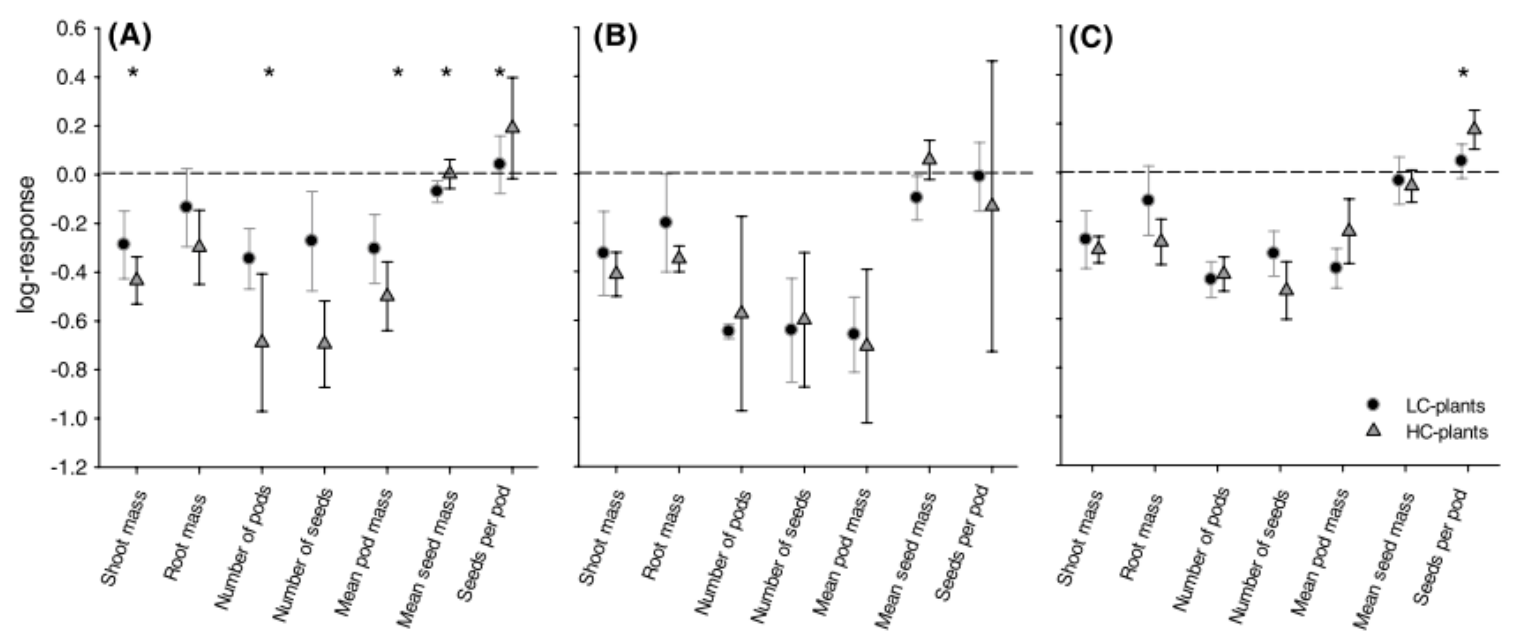

Figure 3.2 Log-response ratios for vegetative and generative traits of $\mathrm{HC}$ - and LC-plants for the influence of a interspecific competition by corn, b intercyanotypic competition, and c intra-cyanotypic competition (means $\pm \mathrm{CI}$ ). Confidence intervals not overlapping the zero line indicate significant responses. Asterisks indicate significant differences between LC- and HCplants based on a paired t test $(\mathrm{P}<0.05)$.
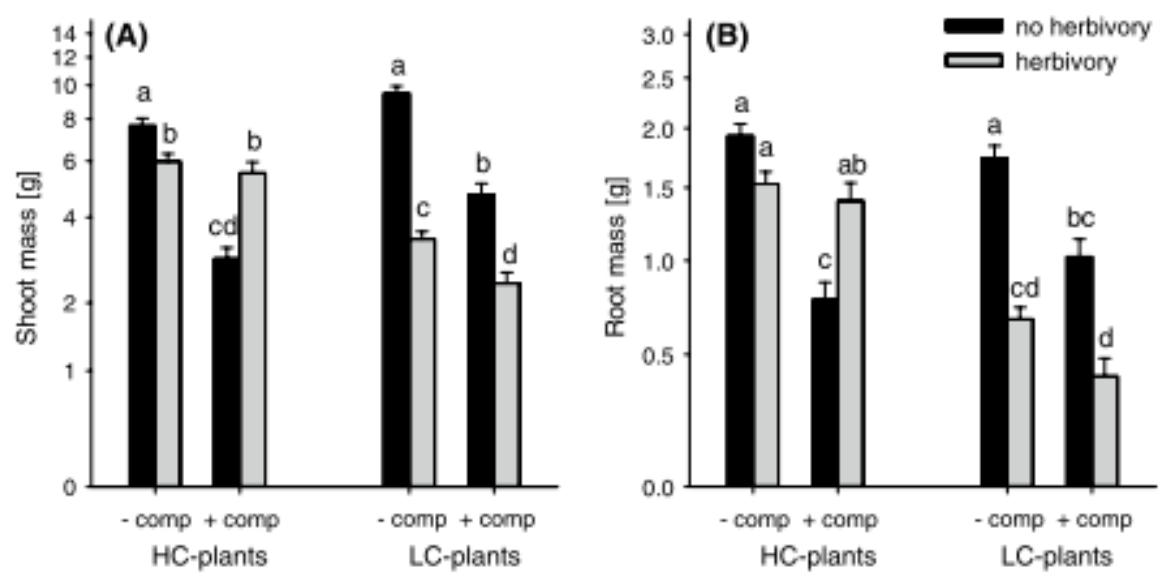

Figure 3.3 Effects of inter-cyanotypic competition, cyanotype, and herbivory on vegetative traits of lima beans (mean $\pm \mathrm{SE}$ ). Different letters indicate significant differences between means following post hoc tests (Tukey's, $\mathrm{P}<$ 
0.05). Note that only ANOVAs with a significant interaction between competition and herbivory are followed by post hoc tests. Note that the y-axes are on a $\log$ scale
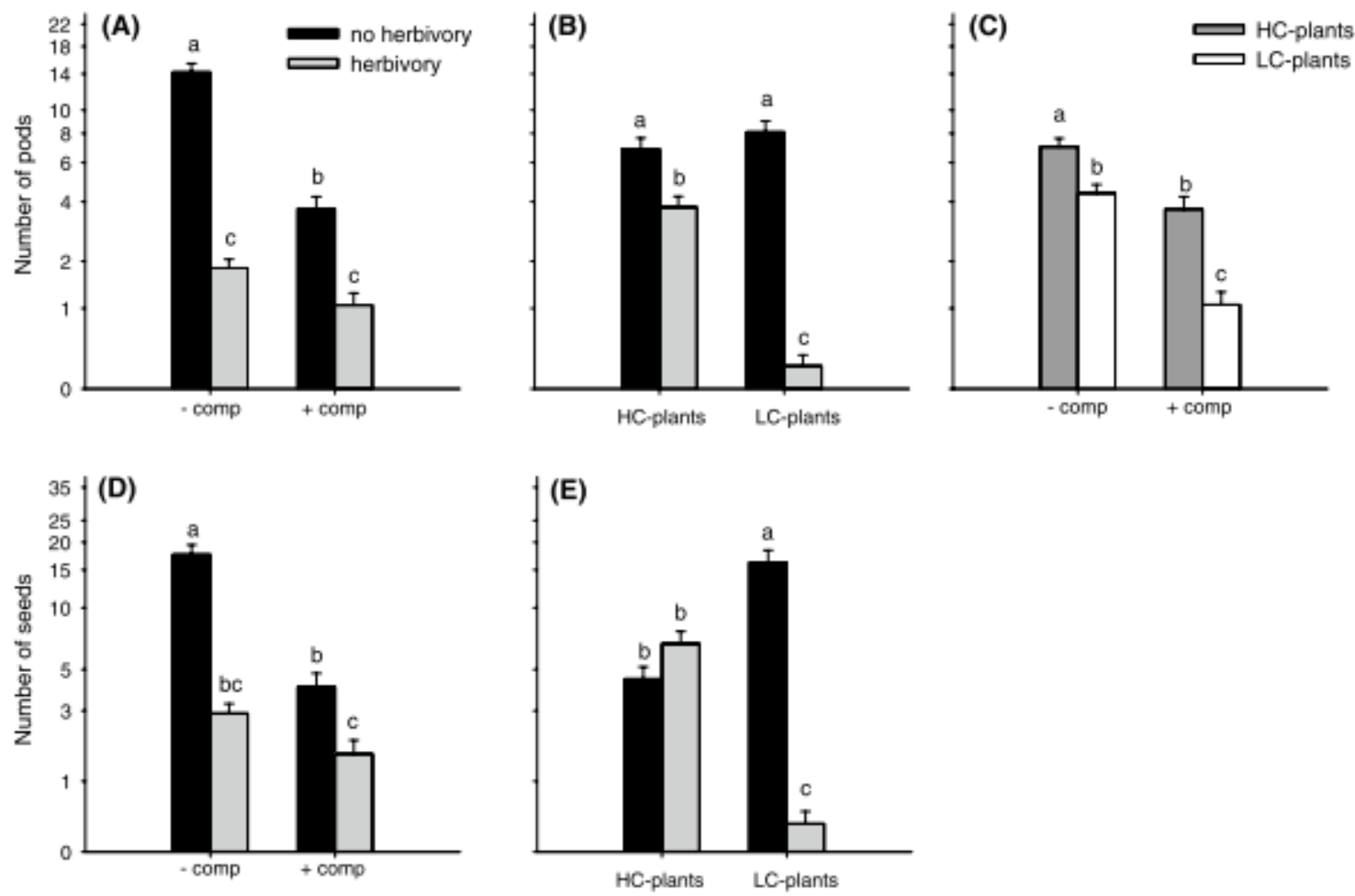

Figure 3.4 Effects of inter-cyanotypic competition, cyanotype and herbivory on generative traits of lima beans (mean $\pm \mathrm{SE}$ ). Different letters indicate significant differences between means following post hoc tests (Tukey's, $\mathrm{P}<$ 0.05). - comp without competition, +comp with competition. Note that the $y$ axes are on a log scale. 

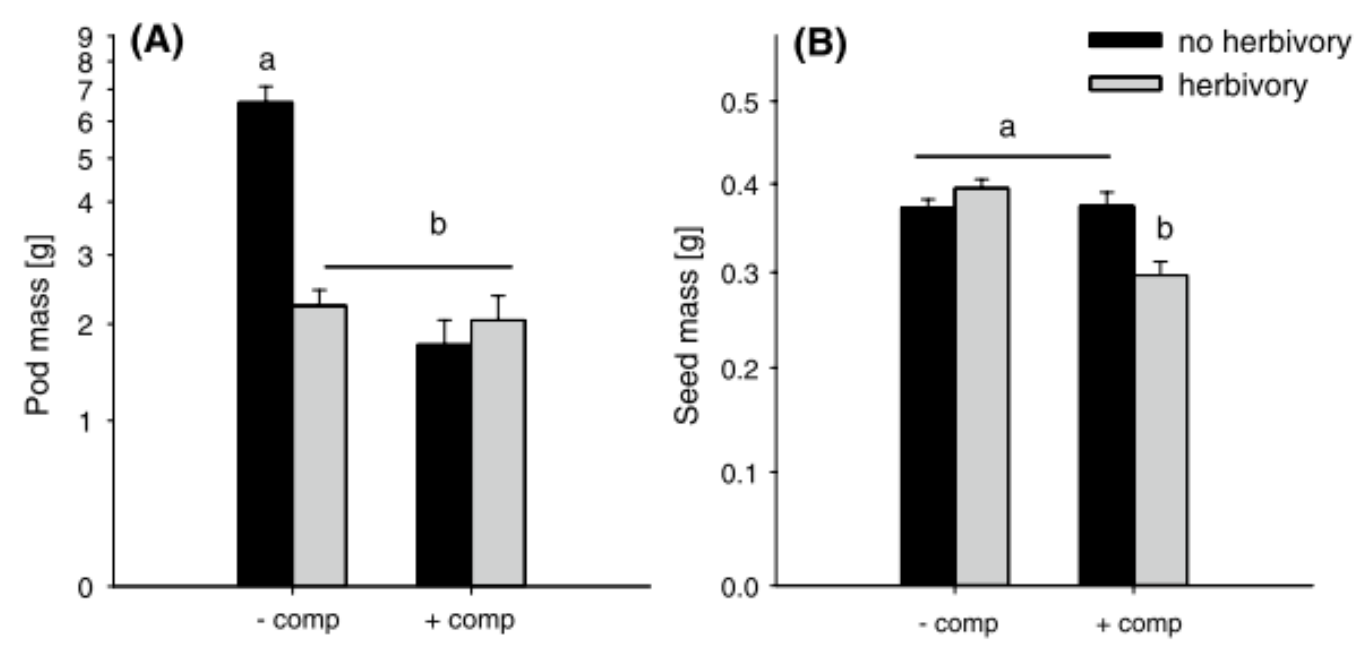

Figure 3.5 Effects of inter- cyanotypic competition and herbivory on generative traits of the HC-genotype of lima beans (mean $\pm \mathrm{SE}$ ). Different letters indicate significant differences between means following post hoc tests (Tukey's, $\mathrm{P}<0.05$ ). - comp without competition, +comp with competition. Note that the y-axes are on a log scale.
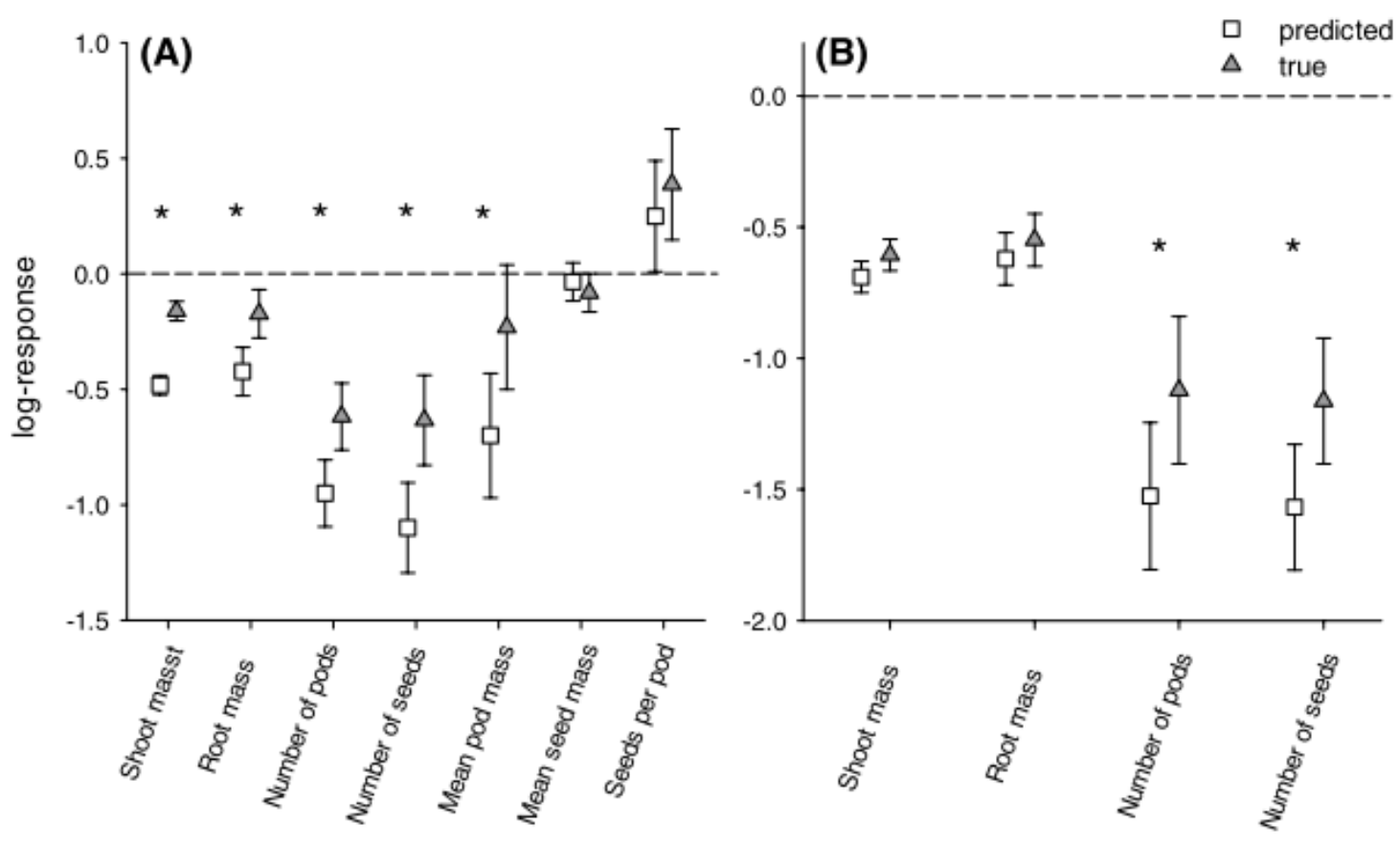
Figure 3.6 Comparison of predicted (calculated from separate responses to competition and herbivory) and observed responses to the simultaneous effect of herbivory and competition for HC- and LC-plants of lima bean (mean \pm CI). Confidence intervals not overlapping the zero line indicate significant responses. Asterisks indicate significant differences between predicted and true responses (ANOVA, method of calculation, $\mathrm{P}<0.05$ ) 


\section{Chapter 4: Which plant organs are most important to defend?}

Published as: Godschalx AL, Stady L, Watzig B, Ballhorn DJ. 2016. Is protection against florivory consistent with the optimal defense hypothesis? BMC Plant Biology: 1-9.

Abstract: Background: Plant defense traits require resources and energy that plants may otherwise use for growth and reproduction. In order to most efficiently protect plant tissues from herbivory, one widely accepted assumption of the optimal defense hypothesis states that plants protect tissues most relevant to fitness. Reproductive organs including flowers and immature fruit, directly determining plant fitness, as well as young, productive leaf tissue thus should be particularly well-defended. To test this hypothesis, we quantified the cyanogenic potential ( $\mathrm{HCNp}$ - - a direct, chemical defense—-systemically expressed in vegetative and reproductive organs in lima bean (Phaseolus lunatus), and we tested susceptibility of these organs in bioassays with a generalist insect herbivore, the Large Yellow Underwing (Noctuidae: Noctua pronuba). To determine the actual impact of either florivory (herbivory on flowers) or folivory on seed production as a measure of maternal fitness, we removed varying percentages of total flowers or young leaf tissue and quantified developing fruit, seeds, and seed viability. Results: We found extremely low HCNp in flowers $\left(8.66 \pm 2.19 \mu \mathrm{mol}_{\mathrm{CN}} \mathrm{g}^{-1}\right.$ FW in young, white flowers, $6.23 \pm 1.25 \mu \mathrm{mol} \mathrm{CN}^{-} \mathrm{g}^{-1} \mathrm{FW}$ in mature, yellow flowers) and in pods (ranging from $32.05 \pm 7.08$ to $0.09 \pm 0.08 \mu \mathrm{mol} \mathrm{CN} \cdot \mathrm{g}^{-1} \mathrm{FW}$ 
in young to mature pods, respectively) whereas young leaves showed high levels of defense (67.35 $\left.\pm 3.15 \mu \mathrm{mol} \mathrm{CN} \mathrm{Cg}^{-1} \mathrm{FW}\right)$. Correspondingly, herbivores consumed more flowers than any other tissue, which, when taken alone, appears to contradict the optimal defense hypothesis. However, experimentally removing flowers did not significantly impact fitness, while leaf tissue removal significantly reduced production of viable seeds. Conclusions: Even though flowers were the least defended and most consumed, our results support the optimal defense hypothesis due to i) the lack of flower removal effects on fitness and ii) the high defense investment in young leaves, which have high consequences for fitness. These data highlight the importance of considering plant defense interactions from multiple angles; interpreting where empirical data fit within any plant defense hypothesis requires understanding the fitness consequences associated with the observed defense pattern.

Introduction: Toxic, tough, or unpalatable compounds protect plant tissues against herbivory, making plant defense the gatekeeper mediating food web energy flow. Plant defense patterns vary between plant species and within individuals. To explain this variation, several plant defense theory hypotheses aim to predict the factors driving plant defense patterns (Stamp, 2003). The optimal defense hypothesis (ODH) predicts defense patterns that confer the greatest fitness benefit to the plant and mitigate energetic costs 
(McKey, 1974). One cost-saving strategy is differentially protecting organs within the plant, allocating more defense compounds to organs with highest impacts on fitness. Organs predicted to have a particularly high fitness role include reproductive organs as well as active and young vegetative structures that provide the current and future source of photosynthates required for reproduction (Ohnmeiss \& Baldwin, 2000; Stamp, 2003; Barto \& Cipollini, 2005a; Heath et al., 2014). Testing within-plant defense allocation according to $\mathrm{ODH}$ predictions requires understanding 1) the value of each plant part, 2) the benefit of defending that organ, and 3) probability that organ will be attacked (Zangerl \& Bazzaz, 1992). Using these parameters, the aim of this study is to determine whether a plant well-characterized to produce high levels of chemical defense in leaf tissue also invests defensive compounds in flowers, and the role of such pattern according to the ODH.

Plants use many compounds for defense that require amino acids or carbonbased molecules as precursors as well as energy-demanding enzymatic pathways to be produced. Because these precursors would otherwise be used to synthesize proteins or structural compounds, chemical defenses can be costly to the plant (Simon et al., 2010; Ballhorn et al., 2014a). In lima bean (Fabaceae: Phaseolus lunatus), one such energetically costly defense, cyanogenesis, requires proteinogenic amino acids and several enzymes to produce cyanogenic precursors (cyanogenic glucosides). For example, the 
cyanogenic glucosides in lima bean, linamarin and lotaustralin are synthesized from valine and isoleucine (Butler \& Conn, 1964; Hahlbrock \& Conn, 1970). When cells are damaged, two enzymes, B-glucosidase and hydroxynitrile lyase, work sequentially to efficiently release cyanide from the cyanogenic glucosides (Frehner \& Conn, 1987; Kakes, 1990; Poulton, 1990; Vetter, 2000; Gleadow \& Møller, 2014a). Taken together, the machinery required to release toxic hydrogen cyanide requires a significant input of nitrogen, which is frequently limited in terrestrial ecosystems. Even legumes, which form a symbiotic relationship with nitrogen-fixing rhizobia face allocation costs due to the photosynthate required to maintain the relationship (Godschalx et al>, 2015a). Thus, efficiently allocating nitrogenrich cyanogenic precursors from the source organs to specific and particularly valuable plant tissues would likely lead to higher fitness (Gleadow \& Møller, 2014a).

Cyanogenesis is an efficient defense against various herbivores, but also incurs costs to the plant in synthesis and transport as well as in ecological interactions (Ballhorn et al., 2005, 2007). To prevent autotoxicity in the intact plant, vacuolar cyanogenic glucosides are spatially separated from apoplastic B-glucosidases, which combine when herbivores rupture cellular barriers (Frehner \& Conn, 1987). However, in the absence of herbivores, when faced with plant-plant competition, investment in extensive cyanogenesis can 
reduce plant fitness (Ballhorn et al., 2014a), reemphasizing the intrinsic costs of this defense. Furthermore, extensive cyanogenesis may make plants more susceptible to fungal pathogens as it has been shown in studies on several cyanogenic plant species such as rubber tree (Lieberei et al., 1989) as well as lima bean (Ballhorn et al., 2010b; Ballhorn, 2011b). To minimize costs, plant cyanogenesis varies among plant organs and in different conditions (Gleadow et al>, 1998; Goodger>et al>, 2004; Ballhorn>et al>, 2009b; Godschalx>et al>, 2015a). In lima bean, the experimental plant used in this study, cyanogenic potential (HCNp) depends on various factors. For example, individuals extensively colonized with nitrogen-fixing rhizobia have higher HCNp than conspecifics without the additional source of nitrogen that rhizobia provide (Thamer et al., 2011; Godschalx et al., 2015a), and within these plants, young leaves are more cyanogenic. In some plants such as Eucalyptus cladocalyx, cyanogenic glucosides are found throughout both vegetative and reproductive structures, and vary temporally resulting from a potential reallocation of cyanogenic resources from leaves to flowering structures (Gleadow \& Woodrow, 2000). Although lima bean is a well-established model plant in chemical ecology, cyanogenesis of flowers and fruit—organs directly associated with plant fitness—has not yet been measured.

Here we test a key assumption of the ODH: that the within-plant distribution of plant defense reflects the plant organs' relevance for fitness. 
To determine quantitative defense investment patterns and resistance to herbivores, we measured cyanogenesis in flower buds, flowers, seed pods as well as in leaves from varying developmental stages, and assessed how much a generalist insect herbivore, the Large Yellow Underwing (Noctuidae: Noctua pronuba) would consume each organ. To determine the impact of florivory on plant fitness (defined as number of viable seeds produced per plant) and to compare any impacts with the fitness consequences of folivory (on young, productive leaves), we experimentally removed different percentages of either flowers $(0,25,50$, and $75 \%)$, or young leaf tissue $(0 \%$, $33 \%, 50 \%$, and $66 \%$ ). Combining measurements of flower and young leaf HCNp with simulated florivory and folivory experiments enables us to determine the fitness value of each type of organ to the plant and benefit of defending them, while bioassays visualize the probability of flowers and leaves being attacked. If simulated folivory impacts fitness, we expect to see high HCNp in young leaf tissue. If removing flowers significantly reduces plant fitness, we expect flowers and pods will have higher HCNp than vegetative plant tissues, consistent with the ODH. Alternatively, if removing flowers has little or no measurable impact on plant fitness, plants with low cyanogenic flowers and fruit will support the optimal defense hypothesis.

\section{Results: Within-plant distribution of chemical defense}


As each organ matured (flower buds, flowers, pods, and leaves), the cyanogenic potential (HCNp) for that organ decreased. The reproductive organs with the highest $\mathrm{HCNp}$ were young pods with $32.05 \pm 7.08 \mu \mathrm{mol} \mathrm{CN}^{-} \mathrm{g}$ ${ }^{1} \mathrm{FW}$, which decreased to almost non-detectable levels of $0.09 \pm 0.08 \mu \mathrm{mol} \mathrm{CN}^{-}$ $\mathrm{g}^{-1} \mathrm{FW}$ as pods developed to intermediate and mature pods, making mature pods that are preparing for senescence the lowest cyanogenic plant organs [Figure 1A, one-way ANOVA: $\mathrm{F}_{1,9}=381.64, p<0.001$; Tukey's HSD, $p<0.05$ ]. These low levels of cyanide are also found in the more mature developmental stages of flowers. In small flower buds, $\mathrm{HCNp}$ is the second highest among reproductive organs, which decreased as flower buds grew larger, and further decreased when flowers first bloomed (white petals), and then mature and change color to yellow. Yellow flower HCNp is not significantly different from the lowest cyanogenic organs (intermediate and mature pods) with $6.23 \pm 1.25$ umol CN- $\mathrm{g}^{-1} \mathrm{FW}$ (Figure 1A). By contrast, young leaves contained the highest concentration of cyanide with an average HCNp of $67.35 \pm 3.15 \mu \mathrm{mol} \mathrm{CN}^{-} \mathrm{g}^{-1}$ FW (Figure 1A). As leaves developed into intermediate and mature stages, HCNp significantly decreased relative to the highly cyanogenic young leaves (Figure 1A, one way ANOVA: $\mathrm{F}_{1,9}=381.64, p<0.001$ ). Intermediate leaves had similar HCNp values as young pods, and mature leaves had HCNp values not significantly different from the lower cyanogenic flower buds and flowers. 
Soluble protein concentration: Similar to $\mathrm{HCNp}$, soluble protein content (an important nutritive trait: (Ganzhorn, 1992)), content in lima bean organs decreased with maturity (Figure 1B). Between organs, protein content differed significantly $\left(\mathrm{F}_{1,9}=21.68, p<0.001\right)$ with young leaves, small flower buds, large flower buds, flowers, and young pods all containing higher protein concentrations than mature leaves, and both intermediate and mature pods (Figure 1B). We found no significant difference in total soluble protein content between young leaves, the most cyanogenic organ, and all flower developmental stages, one of the least cyanogenic organs (Figure 1B). Thus, flowers have the highest nutritive value: low defense, but high protein.

Cafeteria-style feeding trials: Variation in $\mathrm{HCNp}$ among organs resulted in significant differences in fresh weight of food consumed, showing variation in herbivore food choices (one-way ANOVA, $\mathrm{F}_{1,9}=31.369, p<0.001$ ). Insects preferred organs with the lowest $\mathrm{HCNp}$, with the exception of mature pods and leaves (Figure 2). Among the low cyanogenic tissues, herbivores preferred flowers more than any other tissue, followed by large flower buds, and intermediate pods, both of which released $<20 \mu \mathrm{mol} \mathrm{CN} \cdot \mathrm{g}^{-1} \mathrm{FW}$. HCNp decreased as pods developed, but the mature stage pods also began to develop tougher, mechanically defended tissue in preparation for senescence. Both young and mature pods were consumed significantly less than intermediate pods (Figure 2). Compared with any leaf tissue, herbivores in this experiment 
consumed three times more flower tissues (Figure 2).

Plant fitness consequences of florivory: Simulating florivory by removing flowers had no measurable impact on plant maternal fitness. The reproductive output per plant, measured as the number of viable seeds, was not affected by simulated florivory treatments (Figure 3). Removing 0\%, 25\%, $50 \%$, or $75 \%$ of flowers did not significantly affect pod number (one-way ANOVA, $\left.\mathrm{F}_{1,3}=0.466 p=0.707\right)$, total seeds $\left(\mathrm{F}_{1,3}=1.634, p=0.189\right)$, or total viable seeds $\left(\mathrm{F}_{1,3}=2.098, p=0.108\right)$.

Plant fitness consequences of young leaf folivory: Simulating folivory on young leaves did significantly decrease plant maternal fitness. The reproductive output per plant, measured as the number of viable seeds, quantitatively decreased as leaf removal was experimentally increased (Figure 3). Removing $0 \%, 33 \%, 50 \%$, or $66 \%$ of young leaf tissue reduced final pod number (one-way ANOVA, $\left.\mathrm{F}_{1,3}=80.475 p<0.001\right)$, total seeds $\left(\mathrm{F}_{1,3}=77.530, p<0.001\right)$, and total viable seeds $\left(\mathrm{F}_{1,3}=94.261, p<0.001\right)$.

Discussion: In this study we tested one prediction of the optimal defense hypothesis (ODH), which states plants should allocate defense compounds towards tissues that are most relevant for plant fitness (Stamp, 2003). Testing such investment in defense traits across different plant organs requires all organs to rely on the same kind of defense (Zangerl \& Rutledge, 
1996). We show here that lima bean plants accumulate the defensive compounds, cyanogenic glucosides, in all aboveground plant tissues tested (Kakes, 1990; Ballhorn et al., 2005). Comparing the cyanogenic potential (HCNp) of flower buds, flowers, pods, and leaves from several developmental stages, we found that the organs with the highest $\mathrm{HCNp}$ were not the reproductive organs (i.e., flower buds, flowers, and pods), which directly determine plant fitness, but instead were young leaves. These findings are consistent with cyanogenic patterns in Eucalyptus cladocalyx in which young leaves have the highest HCNp among all organs (Gleadow \& Woodrow, 2000). In our study, we conducted feeding trials with generalist insect herbivores to assess the probability that herbivores would attack each organ and found that—corresponding to their low $\mathrm{HCNp}$ and high nutritive value—-the insects preferred flowers among all tested plant organs. In nature, lima bean is attacked by various generalist and specialist herbivores (Ballhorn et al., 2010a, 2013b). We frequently observed noctuid generalist caterpillars feeding during the night on various organs of lima bean plants including all organs tested in this study as well as generalist locusts feeding on the same tissues during the day. Thus, larvae of the generalist noctuid moth species $(N$. pronuba) selected for this study seem suitable for bioassays with lima bean tissues.

According to the ODH, attack risk is one factor that should increase 
defense compound allocation to that organ, and in this case flowers seem to have a high risk for attack but low defense, counter to theoretical predictions (McKey, 1974; Cates \& Rhoades, 1977; Stamp, 2003). To experimentally quantify the benefit to the plant associated with defending flowers we compared the flowers' low HCNp to the fitness value of that organ in simulated florivory experiments. The fitness consequences associated with florivory revealed support for the optimal defense hypothesis because removing flowers does not impact our metric for measurable fitness: viable seed production. Thus, in our system, the number of individual flowers does not critically determine the reproductive output per plant individual.

If removing a portion of total flowers does not reduce seed production, investing resources towards defense compounds in flowers likely does not maximize fitness. In fact, compared to plants with all flowers intact, in our study removing any percent of flowers causes a slight, but not significant, increase in number of pods, total seeds, and viable seeds. This phenomenon has been described in another study on Solanum carolinense, where flower removal designed to simulate weevil damage stimulates mature fruit production (Wise et al., 2008). In Phaseolus vulgaris, a plant species closely related to our experimental plant, removing flowers between day 11 and 20 within the flowering period can increase seed yield (De Moura \& Foster, 1986). Because flowers contribute differentially to final seed yield depending 
on timing within the flowering period (Doust \& Eaton, 1982), we removed a given percentage of flowers continuously throughout the flowering period to exclude any flowering timing effects. Overall, the lack of reduced seed number and viability with varying degrees of simulated florivory helps to explain the low concentrations of cyanogenic glucosides in flowers. In fact, if some florivory stimulates seed production, this could potentially act as a selection against highly cyanogenic flowers. Whether slight florivory is favored or whether cyanogenic glucoside costs outweigh the benefits of defending flowers, the distribution of cyanogenic glucosides we observed suggests that lima bean plants allocate chemical defense to young leaves rather than to reproductive tissues.

High concentrations of cyanogenic glucosides in young leaves as we observed suggests that plants allocate these compounds from the source organs-likely intermediate, fully photosynthetically active leaves (Ballhorn, unpubl. data)—-to the young leaf sinks. Young leaves likely have an important fitness contribution due to their role as future producers of photosynthates important for growth and reproduction. In this line, plants with various levels of simulated folivory produced fewer pods, seeds, and viable seeds in this study in a quantitative damage-response relationship. High protection of young, expanding leaves is a consistent pattern with other studies that test the optimal defense hypothesis (Ohnmeiss \& Baldwin, 2000; Barto \& 
Cipollini, 2005b; Radhika et al., 2008; Alba et al., 2012; Heath et al., 2014). Herbivores attack young leaves $>20$ times more often than more mature leaves (Kursar \& Coley, 2003). Given the risk for attack and value as a potential future carbon source organ, plants often protect young leaves relatively more than mature leaves (Brown et al., 1991; Ballhorn et al., 2008c; Bixenmann et al., 2013). Young leaves of Eucalyptus cladocalyx consistently have the highest concentrations of cyanogenic precursors (Gleadow et al., 1998; Gleadow \& Woodrow, 2000). Terpenoid concentrations are highest in the young leaves of Solidago altissima, which impact capitula mass more than other tissue when removed (Heath et al., 2014). Barto and Cipollini (2005) removed leaves from various developmental stages of Arabidopsis thaliana and also concluded young leaves can be the most valuable plant organ for measurable plant fitness. Our HCNp data in concert with our data showing fitness consequences of removing young leaf area are consistent with the optimal defense hypothesis with fitness-relevant organs, young leaves in this case, being the most cyanogenic.

How do multiple defenses interact to shape organ-specific levels of chemical defense?

In addition to having the highest cyanogenic potential in our study, young lima bean leaves produce the highest quantities of extrafloral nectar and 
volatile organic compounds, both of which are indirect plant defenses, attracting enemies of the plant's herbivores to protect the plant (Radhika et al>, 2008; Godschalx>et al>, 2015a). Frequently plants employ multiple defense strategies, including indirect defenses to protect against their diversity of attackers (Agrawal \& Fishbein, 2006; Turley et al., 2013; Ballhorn et al., 2013a). Among these defenses, tradeoffs between direct and indirect or inducible defenses can be adaptive to conserve resources and maximize fitness, consistent with the optimal defense hypothesis (Zangerl \& Rutledge, 1996; Stamp, 2003; Wäckers \& Bonifay, 2004). Several traits in lima bean trade off with cyanogenesis, including several mechanical, inducible, and indirect defenses (Ballhorn et al., 2013a). Lima bean genotypes with consistently high cyanogenic potential produce less extrafloral nectar, carbon-based volatile organic compounds, and are more susceptible to pathogen attack (Ballhorn et al., 2008b, 2010b, 2013a). The sum of plant defense interactions against attack on all plant parts, including both florivory and folivory, may help explain the distribution of any individual defense compound within plant tissues. For example, phenolic glycosides concentrations in Populus tremuloides leaves were 30\% higher when leaves also contained extrafloral nectaries (Young et al., 2010), which follows the pattern of extrafloral nectar and cyanogenesis in lima bean. This pattern could be consistent with optimal defense predictions to protect against 
different feeding guilds, or if investing resources towards one defense makes that organ important to protect. For example, leaves that secrete extrafloral nectar can serve as a significant resource sink (Ballhorn et al., 2014b), and plants may have higher fitness by protecting the carbon investment. However, this dual protection pattern contradicts the optimal defense hypothesis if investing in multiple defenses is redundant, or if the plant's defenses deter or harm beneficial insects. An example of this occurs in Mentzelia pumila plants, which have trichomes that trap and kill predatory coccinellid beetles (Eisner et al., 1998). Trichome density as a mechanical defense covaries with cyanogenesis, with hook-shaped trichomes expressed in greater frequency in highly cyanogenic lima bean genotypes, putatively as a mechanism to protect tissues against a broader range of herbivores with different feeding strategies (Ballhorn et al., 2013a).Chewing herbivores effectively rupture cellular barriers between enzymes and precursors, but herbivores such as phloem- or cell content feeders that can avoid extensive damage may be more affected by barriers to accessibility, including hookshaped trichomes, or even tissue toughness. In our study, the decreasing HCNp fruits and leaves which goes hand in hand with simultaneously increasing toughness of these organs may further indicate an ontogenetically shift from chemical to mechanical defense. The plant's interacting defense traits and resulting within-plant distribution may be constrained by the 
network of ecological interactions, both with herbivores from various feeding guilds or with beneficial mutualists that can contribute to plant fitness.

\section{Ecological implications of florivory and folivory}

Plant defense distribution throughout various organs likely also depends on the ecological value of protecting those organs. Some interspecific interactions greatly impact plant fitness, such as plant-pollinator or plantmicrobe interactions, which may be significantly compromised by either folivory or florivory. Symbiotic, nitrogen-fixing rhizobia in legume root nodules can consume up to $20-30 \%$ of the plants' total photosynthate pool (Peoples et al., 1986), and intense leaf area removal by folivores can starve other plant organs of carbon when photosynthesis is limited (Tsikou et al., 2013). Quantitative leaf removal also reduces extrafloral nectar secretion, a reward plants use to attract natural predators such as ants (Ballhorn et al., 2014b). Leaf removal, herbivory, and simulated herbivory alter flower size and shape, which not only impacts the energy reserves within the reproductive structure, but also may attract fewer pollinators (Mothershead \& Marquis, 2000; Strauss \& Whittall, 2006; Kessler \& Halitschke, 2009a). Pollen per plant and pollen quality can decrease with leaf consumption (Strauss, 1997). Flower consumption can reduce the strength of the visual or chemical signals that attract pollinators (Gori, 1989). Reduced pollination 
decreases male fitness by limiting pollen transfer (McCall \& Irwin, 2006). By altering rates of outcrossing, florivores can act as a selection pressure for entire mating systems, increasing the frequency of selfing, which can have severe fitness consequences (Penet et al., 2009). Therefore, although we were not able to measure the fitness consequences associated with ecological interactions, protecting various organs with high levels of chemical defense may be partly explained in the context of plants maintaining mutualistic interactions.

Conclusions: In our study, we test the optimal defense hypothesis (ODH) by assessing the cyanogenic potential of reproductive and vegetative organs in a highly cyanogenic plant. By comparing floral cyanogenesis, the risk that generalist herbivores would consume floral tissue, and the fitness value of flowers, we examined the factors expected to affect organ-specific defense: 1) value of organ, 2) benefit of defense, and 3) probability for attack (Zangerl \& Bazzaz, 1992). Within-plant distribution of cyanogenic potential-low in flowers, but highest in young leaves-reflects the fitness relevance of the reproductive and vegetative organs, and is consistent with the within-plant assumption of the optimal defense hypothesis. Our HCNp data show that measuring plant traits in various organs alone does not provide a comprehensive picture of defense resource allocation, but combining plant defense patterns with bioassays and evaluating fitness is a more powerful 
approach to determine whether or not the observed patterns align with any theoretical framework for plant defense. The optimal defense hypothesis continues to be a leading hypothesis because the underlying premise enables many plant trait patterns to maximize fitness in the right context. Although this is not the first call to action requiring a big-picture perspective of how plant defenses interact to shape defense allocation patterns, our data emphasize the role of fitness benefits and consequences shaping plant defense distribution patterns. As we continue to measure empirical patterns in plant defense allocation, it becomes increasingly apparent that the fitness consequences and ecological context are both essential for understanding how, when, and where plants protect themselves.

Methods Plant Cultivation:Lima bean plants (genotype CV 8078, (Ballhorn et al., 2010b)) were cultivated in a greenhouse adjusted to resemble conditions at natural lima bean habitats in Costa Rica $\left(30{ }^{\circ} \mathrm{C} / 24^{\circ} \mathrm{C}, 75-85 \%\right.$ humidity, $14 \mathrm{~h} / 10 \mathrm{~h}$ light/dark photoperiod). Lights in the greenhouse were a combination (1:1) of HQI-BT 400W (Osram) and RNP-T/LR 400W (Radium) lamps with a photon flux density of 550-700 mol photons $\mathrm{m}^{-2} \mathrm{~s}^{-1}$ at table height. Plants were cultivated in plastic pots of $15 \mathrm{~cm}$ in diameter in a 1:1 ratio of potting soil (Fox Farms, Arcata, CA) and sand (grain size $0.5 \mathrm{~mm}$ ). Plants were watered daily and fertilized with $50 \mathrm{ml}$ of a $0.1 \%$ aqueous solution of Flory-3 fertilizer [NPK plus magnesium (\%); 15, 10, 15, +2; 
EUFLOR, Munich, Germany] weekly. To simulate resource allocation patterns that more closely resemble organ tissue development within natural populations, plants were inoculated with $10 \mathrm{~mL}$ liquid culture of a labmaintained rhizobia strain isolated from wild lima bean plants in Costa Rica. Position of plants in the greenhouse was rotated every 3 days to exclude position effects. Feeding experiments and analyses of plant chemical traits were conducted after a cultivation period of 8 weeks.

Insect Rearing :Caterpillars of the Large Yellow Underwing (Noctua pronuba) were used in the feeding trials. This insect species represents an extremely polyphagous herbivore feeding on a broad range of herbaceous and woody plants. Caterpillars were reared from eggs in July 2012 and were fed with non-cyanogenic raspberry leaves to avoid adaptations to cyanide-containing food. Noctua pronuba is an invasive pest insect in the United States. Eggs were collected on private property in Raleigh Hills (DJ Ballhorn, Portland, OR). Neither field work nor collection of caterpillar eggs required permits. Our research is in compliance with all relevant guidelines and/or appropriate permissions.

Cyanogenic Potential (HCNp): The cyanogenic potential (HCNp; total amount of cyanide present accumulated in a given tissue) was quantified for leaves, flower buds, flowers and fruit from different developmental stages. For 
preparation of plant extracts, fresh samples weighed to the nearest $0.001 \mathrm{~g}$ were ground with liquid nitrogen in a pre-cooled $\left(4{ }^{\circ} \mathrm{C}\right)$ mortar and pestle. Plant material was homogenized in $3 \mathrm{~mL}$ ice-cold aqueous $\mathrm{Na}_{2} \mathrm{HPO}_{4}$ solution (67 mmol L-1). Enzymatic hydrolysis of cyanogenic precursors was conducted with specific B-glucosidase isolated from rubber tree (Euphorbiaceae: Hevea brasiliensis), a plant containing the same cyanogenic glucosides (linamarin and lotaustralin) as lima bean. We used enzyme solution adjusted to an activity of 20 nkat. Samples were incubated for $20 \mathrm{~min}$ at $30{ }^{\circ} \mathrm{C}$ in a water bath in closed glass vessels (Thunberg vessels) (Ballhorn et al., 2005, 2006) and the HCNp was quantified by enzymatic hydrolysis of cyanogenic precursors and subsequent spectrophotometric detection of released cyanide at $585 \mathrm{~nm}$ using the Spectroquant ${ }^{\circledR}$ cyanide test (Merck, Darmstadt, Germany)

Soluble Protein Content: Concentration of soluble protein in flower, fruit and leaf samples was quantified according to Bradford (1976) with modifications following Ballhorn et al. (Bradford, 1976; Ballhorn et al., 2007). Bradford reagent (Biorad Laboratories, Munich, Germany) was diluted 1:5 with $\mathrm{ddH}_{2} \mathrm{O}$ and $20 \mu \mathrm{L}$ of each homogenized plant sample was combined with $1 \mathrm{~mL}$ of diluted Bradford solution. Bovine serum albumin (BSA; Fluka ChemieAG, Buchs, Switzerland) at different concentrations was used to create a standard curve. After 5 min incubation time, concentration of protein was 
spectrophotometrically measured at $595 \mathrm{~nm}$. We used the same individual plant extracts for protein measurements as for $\mathrm{HCNp}$ analyses, thus, both parameters were quantitatively attributed to the same sample.

Feeding Trials: Cafeteria-style feeding trials were conducted in Petri dishes (9 cm; N=6 feeding trials) lined with moist filter paper to avoid water loss of samples. Each dish contained one insect herbivore (3rd larval stage). Preweighed plant leaf samples (leaf discs, $1 \mathrm{~cm}$ in diameter), flower buds, flowers and fruits (large fruits were presented in form of discs cut out with a cork borer; $1 \mathrm{~cm}$ in diameter) were offered simultaneously to the insects over a time period of $2 \mathrm{~h}$. Plant fresh material consumption was determined by reweighing the plant samples. A control set of each organ was weighed and reweighed after $2 \mathrm{~h}$ to control for potential evaporation and change in mass due to non-consumptive effects. As we did not observe detectable weight loss for any of the fresh plant samples in this control we did not consider spontaneous evaporation as a factor potentially affecting our results.

Flower Removal Effects on Fitness: To assess fitness consequences of florivory, a given percentage of flowers were mechanically removed throughout the experiment from each plant to create four treatments: $0 \%$ flowers removed, $25 \%$ flowers removed, $50 \%$ flowers removed, and $75 \%$ flowers removed. Flowers were removed at a medium flower bud stage and 
treatments were repeated every three days throughout the flowering period. The experimental duration covered the whole period from the formation of the first inflorescence to the opening of the last flowers. After seeds matured fully, the pod and seed production for each plant was counted and seed viability per plant was determined by germinating seeds on moist paper towels until a healthy radicle developed.

Young Leaf Removal Effects on Fitness: To assess fitness consequences of folivory, developing young trifoliate leaves were mechanically damaged to create four treatments: 0\%, 33\%, 50\%, and 66\% leaf area removal (Fig. 4). These percentages were modified from the ones utilized in the flower removal experiments based on the nature of the trifoliate leaf (Figure 4). Initial leaf area removal was applied when plants developed their first fully unfolded secondary leaf, and experimental leaf tissue removal was continuously applied (once per week) to newly developed, but unfolded leaves throughout the experimental period. Maternal fitness including pod number, seed number and viability were measured as in flower removal experiments.

Statistical Analyses: Data for HCNp, protein content, cafeteria experiment feeding trials, and flower removal experiments were all analyzed using OneWay ANOVA tests followed by Tukey's post hoc tests in R Studio. 

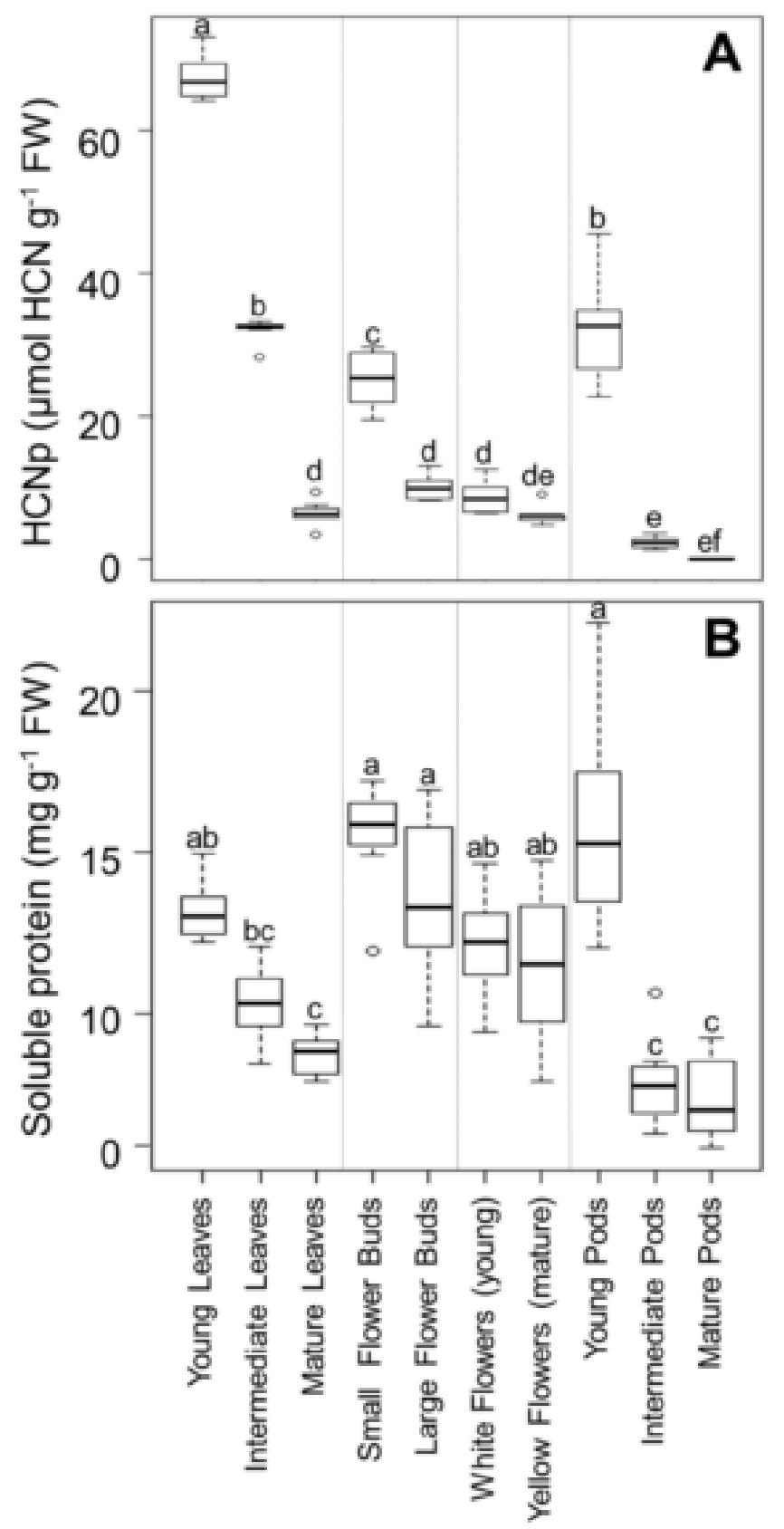

Figure 4.1 Cyanogenic potential (HCNp; a) and soluble protein content (b) of different lima bean organs. Boxplots show median plant trait values in bold with rectangles representing the interquartile range from the 1 st to the $3 \mathrm{rd}$ quartile. Whiskers show minimum and maximum values. Letters indicate significant differences according to posthoc analyses (Tukey'sHSD; $p<0.05$ ) after one- way ANOVA, $\mathrm{N}=8$. 


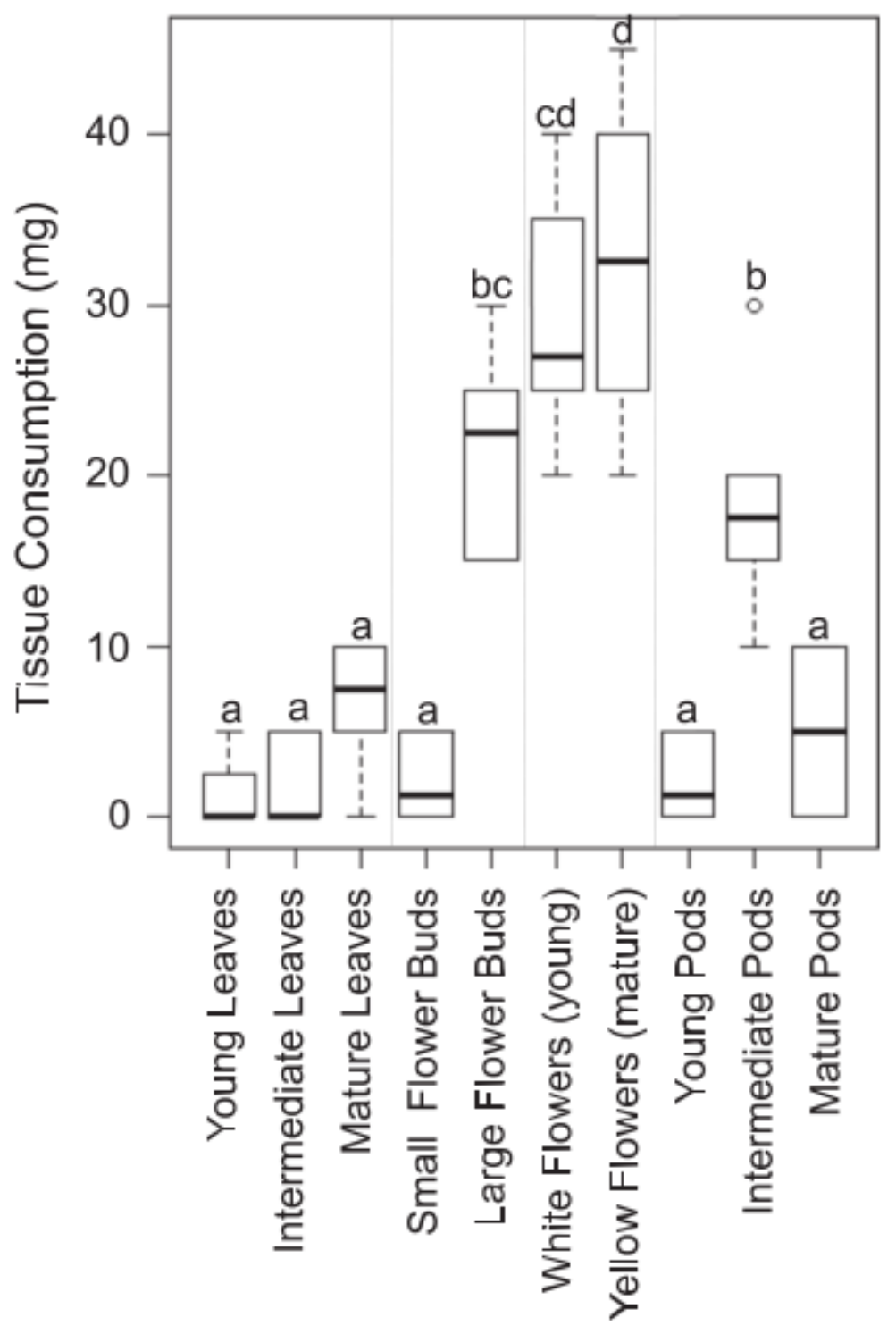

Figure 4.2 Tissue consumed by generalist herbivores. Different plant organs were offered to Noctua pronuba larvae in choice feeding trials and tissue consumption was determined. Boxplots show median tissue consumption by in bold with rectangles representing the interquartile range from the 1st to the 3rd quartile. Whiskers show minimum and maximum values. Letters indicate significant differences according to posthoc analyses (Tukey's HSD; $p$ $<0.05$ ) after one-way ANOVA, $\mathrm{N}=6$ feeding trials 

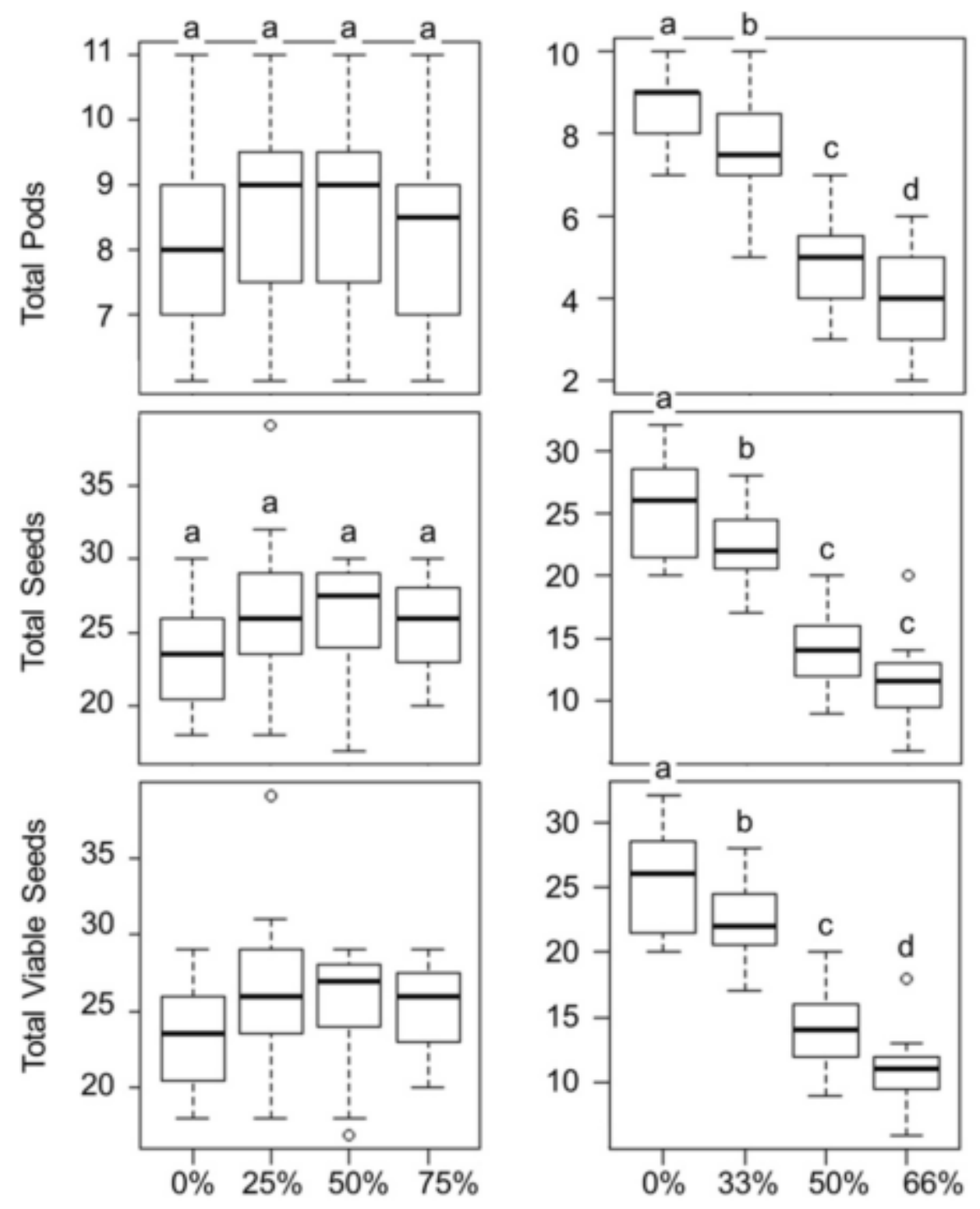

Flowers Removed

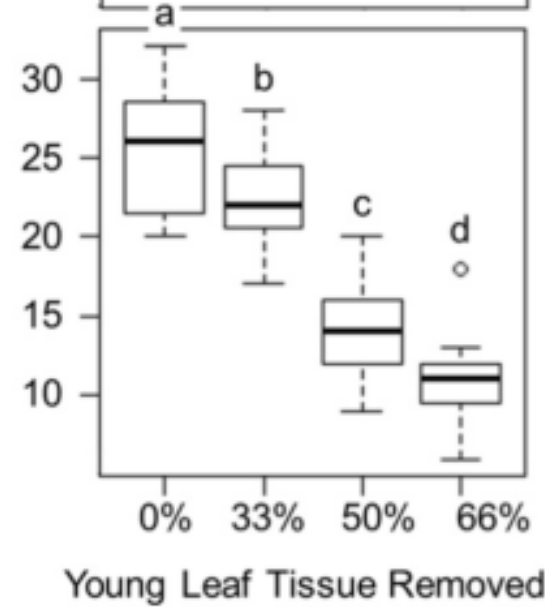

Figure 4.3 Pod and seed production following simulated florivory or folivory. Pod and seed production as well as the number of viable seeds of lima bean plants with different percentages of either flower or young leaf tissue removal were quantified. Tests for differences between flower removal treatments from one-way ANOVAs: total pods, $\mathrm{p}=0.707$, total seeds, $\mathrm{p}=0.189$, and viable seeds, $\mathrm{p}=0.108, \mathrm{~N}=20$. Tests for differences between young leaf tissue removal treatments from one- way ANOVAs: total pods, $\mathrm{p}<0.001$, total seeds, $\mathrm{p}$ $<0.001$, and viable seeds, $\mathrm{p}<0.001, \mathrm{~N}=20$. Boxplots show median values in bold with rectangles representing the interquartile range from the 1 st to the 
3rd quartile. Whiskers show minimum and maximum values. Letters indicate significant differences according to posthoc analyses (Tukey'sHSD; $p$ $<0.05)$
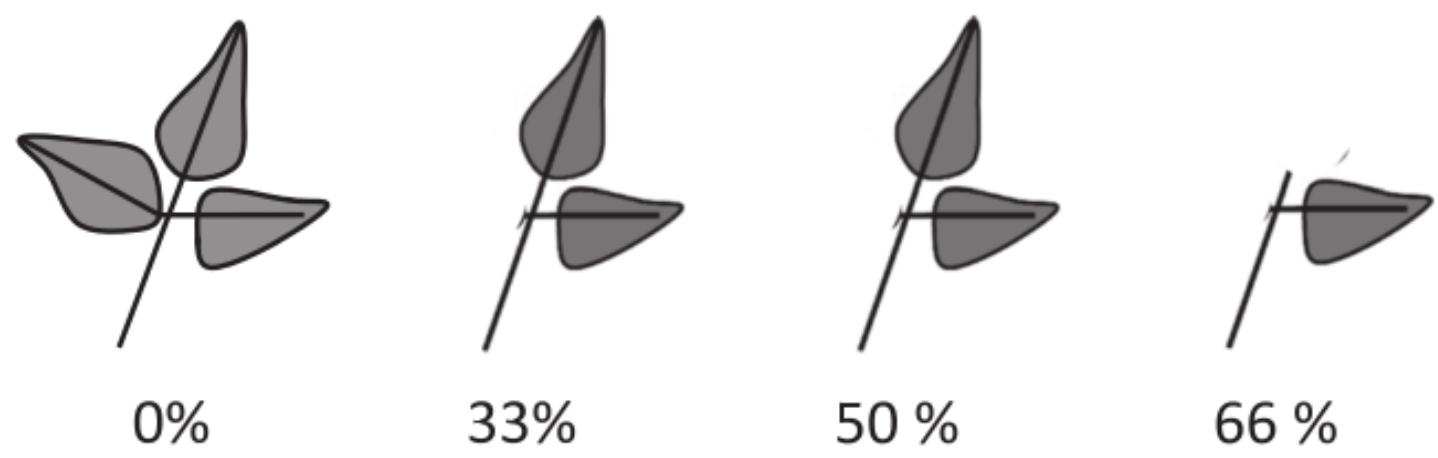

$33 \%$

$50 \%$

$66 \%$

Figure 4.4 Simulated folivory treatment experimental design. Four treatment groups with different percentages of young leaf tissue removal were established as depicted. 


\section{Chapter 5: Cyanotypes differ in rhizobia colonization}

Submitted for publication as: Host plant cyanotype determines degree of rhizobial symbiosis. Godschalx AL, V Tran, DJ Ballhorn. Under review at Ecosphere.

Abstract: Plants with nitrogen-fixing bacteria, such as legumes with rhizobia, can tap the atmospheric nitrogen pool to obtain resources for defense compounds. Cyanogenesis, a nitrogen-based plant defense against herbivores, increases in response to rhizobial colonization, but depends on plant genotype. Here we tested whether genotypic differences in host plant cyanogenesis influence symbiotic reliance on nitrogen-fixing rhizobia. Using thin, clear soil containers, we counted nodules on live root systems of distinct high (HC) and low (LC) lima bean (Phaseolus lunatus) cyanotypes across the duration of an 8-week study. We measured changes in cyanogenic potential $(\mathrm{HCNp})$ and protein content to reveal quantitative interactions between nodule number and leaf chemical traits. HC plants maintained consistently more nodules than LC plants. Including both cyanotypes, nodule number correlated positively with $\mathrm{HCNp}$, but negatively with foliar protein content. However, within both cyanotypes, the significant interactions between nodule number and plant traits disappeared except for foliar protein in $\mathrm{HC}$ plants, which decreased in plants with more nodules. Our results imply that while genotypes with higher levels of nitrogen-based defense invest in more in the 
rhizobial partner, the costs involved in the maintaining the symbiosis may cause resource allocation constraints with the plants' primary nitrogen metabolism.

Keywords: Cyanogenesis, rhizobia, plant defense, genotype, symbiosis, nitrogen-fixation, mutualism, lima bean, Phaseolus lunatus

Introduction: Plant defenses emerged from a long coevolutionary history with herbivores and pathogens (Ehrlich \& Raven, 1964), but also by coevolving alongside beneficial symbiotic partners that influence plantherbivore interactions (Weber \& Agrawal, 2014). Mutualists that aid plants in defense include predators, but also nutrient-provisioning microbes that enhance the availability of certain resources for plant growth and defense (Herms \& Mattson, 1992; Stamp, 2003; Thamer et al>, 2011). In particular, under nitrogen-limited conditions, which are widespread in terrestrial ecosystems (Vitousek et al., 2002), microbes facilitating resource acquisition can mediate plant-insect interactions (Pineda et al., 2010; Thamer et al., 2011). Symbioses between plants and nitrogen-fixing microbes range from loose associations with rhizobacteria in the soil (Dean et al., 2009, 2014; Pineda et al., 2010; Algar et al., 2014; Pangesti et al., 2015c) to highlyregulated interactions within root nodule, involving substantial exchange of resources, which have evolved in multiple taxa of plants and microbes (Vessey et al., 2004; Kempel et al., 2009a). Receiving symbiotic nitrogen in 
exchange for photoassimilates can enable plants to increase growth and defense traits simultaneously, even for relatively costly nitrogen-based defenses (Thamer et al., 2011). This is a significant advantage as producing such costly defense compounds can reduce plant-plant competitive ability and fitness (Herms \& Mattson, 1992; Marak et al., 2003).

Cyanogenesis, the release of toxic hydrogen cyanide from wounded cells, is one of those costly nitrogen-based defenses. In several species, including our experimental plant in this study, lima bean (Phaseolus lunatus), the total amount of cyanide-containing precursors in a given tissue (cyanogenic potential; $\mathrm{HCNp}$ ) is mostly constitutive and varies by genotype, resulting in either high or low levels of cyanogenesis (Ballhorn et al., 2013a; Kautz et al., 2014). High expression can incur both biochemical and ecological costs (Ballhorn et al., 2008b, 2010b). Biochemically, beyond producing cyanide-containing compounds (glucosides) from proteinogenic amino acids, two enzymes, B-glucosidases and hydroxynitrile lyases, work sequentially to efficiently release cyanide from these precursors (Frehner \& Conn, 1987; Kakes, 1990; Poulton, 1990; Vetter, 2000; Gleadow \& Møller, 2014b). Further resources are required for transport and storage as vacuolar cyanogenic glucosides are spatially separated from apoplastic B-glucosidases to prevent autotoxicity (Frehner \& Conn, 1987). Ecologically, cyanogenesis is costly as free cyanide interferes with the function of metal-containing enzymes, 
including enzymes critically involved in resistance to pathogens (Ballhorn et al., 2010b). Consequently, highly cyanogenic plants are generally welldefended against herbivores (Ballhorn et al., 2005), but weakly-defended against pathogens (Lieberei et al., 1989; Ballhorn et al., 2010b). Taken together, the associated ecological consequences and biochemistry involved in releasing cyanide make cyanogenesis a relatively costly plant defense, implying potential for $\mathrm{HC}$ and LC cyanotypes to have different nitrogen requirements. However, cyanotype-driven differences in symbiotic investment to obtain fixed nitrogen has not been previously explored.

While rhizobia may alleviate nitrogen-related costs of defense, maintaining this beneficial relationship introduces another set of physiological and ecological costs. Rhizobia can consume $16-30 \%$ of a plant's total photosynthate pool (Peoples et al., 1986; Kaschuk et al., 2009) and require plant-synthesized essential amino acids for their own metabolism and nitrogen-fixation (Lodwig et al., 2003). The cost for plants to increase nodule numbers includes nodule biomass and metabolic demand to maintain nitrogenase activity, both of which increase carbohydrates, which results in stimulating photosynthetic rates (Kaschuk et al. 2009). Despite increased carbon assimilation, rhizobial carbon demands impact several plant traits, including indirect defense via predator recruitment (Godschalx et al>, 2015a), an additional mutualism posing a carbon sink. Mutualistic carbon sinks play 
an important role in terrestrial carbon cycles (Pringle, 2015b), implying that increasing degree of rhizobia colonization to obtain nitrogen is not trivial.

Despite greater carbon sink and ecological costs, plant cyanotypes with constitutively high levels of cyanogenesis that require large inputs of nitrogen for defense could exert a pressure for plants to form more nodules. Here we tested for quantitative differences in rhizobia colonization between HC and LC lima bean cyanotypes and differential responses in leaf chemical phenotype, including HCNp and soluble protein content to assess overall leaf quality. We collected all three metrics weekly—nodule number, $\mathrm{HCNp}$, and protein content—over the course of a two-month study using a nondestructive method for nodule-counting through clear, thin soil containers. If costs of direct chemical defense impose demand for stronger rhizobial association (measured as nodule number), we expected more nodules would form on roots of $\mathrm{HC}$ compared with LC plants. If leaf phenotype is directly influenced by nitrogen available from symbiosis, we would expect a positive correlation between nodule number and both nitrogen-containing traits. While nitrogenfixing symbioses benefit plant productivity and provide a competitive edge in costly defense investment, understanding the factors facilitating or limiting the degree to which plants engage in this symbiotic exchange remains limited. Here we used two different cyanotypes of the same plant species to 
test the impact of investment into nitrogen-based defense on the legumerhizobia relationship.

Materials and Methods: Experimental setup: To determine rhizobia colonization differences between plant cyanotypes, we used lima bean (Fabaceae: Phaseolus lunatus L.) genotypes previously established as high (HC) or low (LC) cyanotypes based on consistent cyanogenic potential (HCNp) (Ballhorn et al., 2008b). These accessions, HC_8078 and LC_8071 were provided by the Institute of Plant Genetics and Crop Plant Research (IPK) in Gatersleben, Germany. Six seeds per cyanotype were germinated on moist paper towels. Once germinated, lima bean plants were individually planted $0.5 \mathrm{~cm}$ below the substrate surface level (greenhouse mix \#3, SunGro Horticulture ${ }^{\circledR}$, Bellevue, WA) in $15 \mathrm{~cm}$ x $20 \mathrm{~cm}$ x $1.25 \mathrm{~cm}$ clear plastic containers wrapped in sheets of aluminum foil to block light, thus simulating belowground conditions. Soil containers were custom-designed to facilitate rhizobia nodule counts on the intact root system. Plants were watered daily with no additional nutrient solutions and cultivated under greenhouse conditions according to (Ballhorn et al., 2014a) at Portland State University (Portland, Oregon, USA) from March to April 2015.

Rhizobia inoculation: To identify rhizobia, several nodules were surfacesterilized, lysed, and plated to isolate colonies before the $16 \mathrm{~S}$ gene was 
sequenced using 27f/1492r(I) primers. Using geneious software, colonies were identified as Bradyrhizobium elkanii (Accession DJB1033-Ballhorn Lab, Portland State University). Inoculum was prepared by grinding 10 nodules, $0.5-5 \mathrm{~mm}$ in diameter, with a micropestle in a $1.5 \mathrm{~mL}$ centrifuge tube, and suspending the slurry in $600 \mathrm{~mL} \mathrm{H}_{2} \mathrm{O}$. Both cyanotypes were inoculated with rhizobia once seedlings developed at least two true leaves by pouring $50 \mathrm{~mL}$ of rhizobia inoculum on the soil at the base of each seedling. Two weeks after inoculation, all plants showed root nodules.

Plant trait analysis: Nodulation was quantified as total nodule number per root system display. All five surfaces of the clear, thin soil containers included in the root system display, including both sides, both narrow edges, and the narrow base, enabling most of the plant's root system to be included in the survey. Nodule assessments took place weekly for eight weeks. The same collection schedule was followed for leaf trait determination in order to relate rhizobia nodule counts to the quantitative expression of chemical leaf traits. $\mathrm{HCNp}$ was quantified using the Spectroquant ${ }^{\circledR}$ cyanide test (Ballhorn et al., 2005). Briefly, leaves were removed, and three leaf punches from each individual leaf were weighed to the nearest $0.001 \mathrm{~g}$, ground with a mortar and pestle at $4^{\circ} \mathrm{C}$ in $2 \mathrm{~mL}$ ice-cold $\mathrm{Na}_{2} \mathrm{HPO}_{4}$ buffer and centrifuged. Samples were analyzed for HCNp through enzymatically hydrolyzing cyanogenic precursors in gas-tight glass Thunberg vessels and spectrophotometrically 
assaying released cyanide at $585 \mathrm{~nm}$ (Ballhorn et al., 2005). Foliar soluble protein was quantified from the same leaf extracts; using Bradford's reagent and a calibration curve from $50 \mu \mathrm{g} / \mathrm{mg}$ to $1000 \mathrm{\mu g} / \mathrm{mg}$ bovine serum albumin (Amresco, Solon, OH, USA), soluble protein was measured spectrophotometrically at 595nm (Bradford, 1976).

Statistical Analysis: Weekly quantified nodulation, HCNp, and protein content were all analyzed with repeated-measures ANOVAs with cyanotype and time as factors. Relationships among trait means in response to nodule number means were analyzed with a linear model to determine significant relationships and Pearson's coefficients. All analyses were conducted using the software R (version 3.0.2) (R Core Team, 2016).

Results: Rhizobia colonization varied greatly by cyanotype in repeated nodule counts over an experimental period of two months. On average, $\mathrm{HC}$ plants formed significantly more nodules than LC plants $\left(F_{1,10}=21.27\right.$, $p<0.001$; Figure 1A). For any given sampling date, HC plants maintained consistently higher numbers of nodules $\left(F_{6,60}=20.72, p<0.001\right)$, with this lead ranging from 56 to 143 more mean nodules than LC plants. Nodule number varied because of significant interaction effects of cyanotype and sampling date $\left(F_{6,60}=2.64, p<0.05\right)$. 
To test the effects of rhizobia colonization on aboveground plant traits, we measured $\mathrm{HCNp}$ and soluble protein content in leaves of a defined developmental stage from $\mathrm{HC}$ and LC plants. In all cases, $\mathrm{HC}$ leaves produced significantly higher HCNp than LC leaves $\left(F_{1,10}=329.4, p<0.001\right.$; Figure 1B), however, HC leaves contained significantly less average protein than LC leaves $\left(\mathrm{F}_{1,10}=13.44, \mathrm{p}<0.05\right.$; Figure $\left.1 \mathrm{C}\right)$. Sampling date significantly affected both cyanogenic potential $\left(F_{6,60}=46.09, p<0.001\right)$ and soluble protein $\left(F_{6,60}=15.461, p<0.001\right)$. Further, significant interaction effects between sampling date and cyanotype affected $\operatorname{HCNp}\left(F_{6,60}=29.06, p<0.001\right)$, but not protein content $\left(F_{6,60}=1.739, p=0.128\right)$. Both cyanotypes were flowering on May 17th, which corresponded with varying expression of plant traits (Figure 1).

To test for quantitative relationships among leaf chemistry in relation to nodule number across and within cyanotype, we regressed chemical trait averages for each plant replicate against nodule number averages for that same plant individual to test for significant correlations. We found a significant positive relationship between nodule number and $\mathrm{HCNp}$ when we included both cyanotypes $\left(F_{1,10}=15.2, p=0.003\right.$, adjusted $\mathrm{R}^{2}=0.564$; Figure $\left.2 \mathrm{~A}\right)$. This positive correlation between nodule number and $\mathrm{HCNp}$ did not hold true for within-cyanotype correlations. Within HC plants only, HCNp did not form a significant correlation with nodule number, and the slope of the trendline 
was slightly negative $\left(F_{1,4}=0.834, p=0.413\right.$, adjusted $\left.\mathrm{R}^{2}=-0.034\right)$. Within LC plants, the positive trendline was not significant $\left(F_{1,4}=3.817, p=0.122\right.$, adjusted $\left.\mathrm{R}^{2}=0.337\right)$.

Foliar protein content also responded to increasing nodulation. Including both HC and LC plants, as plants formed greater numbers of nodules, protein content significantly decreased $\left(F_{1,10}=21.67, p<0.001\right.$, adjusted $\mathrm{R}^{2}=0.653$;

Figure 2B). Unlike HCNp, which did not form a significant correlation within either cyanotype, a significant negative correlation between nodule number and leaf protein was present within $\mathrm{HC}$ plants $\left(F_{1,4}=20.25, p=0.01\right)$. By contrast, such correlation was not significant within LC plants, which showed a positive trendline between nodules and protein content $\left(F_{1,4}=3.542\right.$, $p=0.133$, adjusted $\left.\mathrm{R}^{2}=0.337\right)$. Overall, cyanotypes invest differentially in foliar protein levels in addition to differentially investing in nodulation.

Discussion: Incorporating symbiotic interactions into patterns explaining plant secondary metabolism has been an important challenge in understanding plant ecology because the advantages afforded by the relationship can drastically influence resource allocation patterns (Kempel et al., 2009a; Heath et al., 2014). Here we show how the legume-rhizobia symbiosis interacts with leaf trait expression quantitatively in high (HC) and low (LC) cyanotypes of lima bean. While HC plants formed more root nodules 
and produced constitutively higher HCNp than LC plants, this positive relationship between nodulation and cyanogenesis did not result in quantitatively higher $\mathrm{HCNp}$ within either cyanotype. These data support our hypothesis that cyanotype may have played a role in selecting for the degree of rhizobial colonization based on constitutive nitrogen demands inherent in cyanogenesis. Surprisingly, our data do not support degree of nodulation quantitatively benefitting defense phenotype. While nodule number did not translate into an increase in cyanogenic potential (HCNp), $\mathrm{HC}$ plants expressed quantitatively less soluble protein in plants with greater nodule numbers. By contrast, LC plants formed fewer nodules overall and foliar protein was not constrained by a negative correlation with nodule number. Our findings suggest that symbiotic investment plays a role in plant defense and nutritive phenotype, but also that genotypic defense levels may simultaneously shape the plant's obligatory investment in maintaining the symbiosis.

\section{Genotypic nitrogen requirements and nodule formation}

Our hypothesis, that $\mathrm{HC}$ plants require more fixed nitrogen for cyanogenesis and would therefore form more nodules than LC plants, was supported by our nodulation data. In another system with polymorphic cyanogenesis, Trifolium repens, acyanogenic strains did not form more nodules than cyanogenic 
strains (Kempel et al., 2009a). However, the nature of cyanogenesis in $T$. repens is qualitative with presence or absence of either cyanogenic glucosides or B-glucosidases, which may impose different resource demands compared to the quantitative variation in the lima bean system, with LC plants that are cyanogenic but at lower levels than HC plants (Ballhorn et al., 2005). If degree of colonization depends on plant nitrogen availability and demand, one potential mechanism for differential nodulation could involve the autoregulated negative feedback loop inhibiting further nodulation. Autoregulation of nodulation involves an interplay of root- and shoot- derived signals in the presence of excess soil nitrate (Oka-Kira \& Kawaguchi, 2006). While the chemical nature of these signals are still largely unknown (Kouchi et al., 2010), our data may present evidence for shoot-derived signals to be differentially regulated in high and low cyanotypes based on nitrogen requirements. Signals being received or sent from cyanogenic tissue may interact with cyanotype-specific leaf chemistry.

\section{Putative mechanisms for nodule differences between cyanotypes}

Nodule formation and regulation is a highly controlled process, involving crosstalk of several plant hormones, plant signaling molecules and bacterial Nod factors (Sun et al., 2006). Therefore, cyanotypic differences in nodule numbers may be connected to cyanotype-specific biology. In addition to a 
myriad of traits regulated differentially between cyanotypes (Ballhorn et al., 2013a), the most obvious difference between cyanotypes would be the presence of high concentrations of cyanogenic glucosides and potentially free cyanide surrounding damaged leaf cells. However, regulation of nodulation is not likely a product of direct cyanide exposure because free cyanide, which is released in the ethylene biosynthesis pathway, acts as a positive feedback loop for further ethylene synthesis (Smith et al., 2000), and ethylene is wellknown to inhibit nodulation (Penmetsa \& Cook, 1997). Inhibiting or reducing ethylene synthesis could be one way HC plants enable more nodules to form if cyanotypes differentially regulate this pathway, although this remains to be tested.

\section{Consequences for plant-microbe interactions}

If autoregulation processes in $\mathrm{HC}$ plants enable greater extent of colonization, are other protective mechanisms also influenced differentially in various cyanotypes, such as host sanctioning and partner choice? Nodule formation is a microbial infection, and interacts with the plant's systemic resistance against pathogenic microbial infection. Common bean plants (Phaseolus vulgaris) with previous fungal pathogen experience in their leaves form fewer belowground associations (Ballhorn et al., 2014c). Nodule number was also reduced in soybean infected with red crown rot (Gao et al., 2012). 
Although lima bean lack $\mathrm{HCNp}$ in root tissues, cyanotype may play a role in belowground interactions due to the foliar constraints in antipathogen defenses (Ballhorn et al., 2010b), which could lead to a similar susceptibility to infection belowground. If this is the case, nitrogen demands for plant physiological processes play an important, understudied role in plantpathogen ecology in legumes. From our data, it is not clear whether colonization degree is more strongly driven by the need for nitrogen or limited by resource tradeoffs between symbiosis maintenance and plant traits.

Is there a nodule number optimum? Nitrogen benefit vs. cost of maintaining nodules

If $\mathrm{HC}$ plants form more nodules to attain sufficient nitrogen for cyanogenesis, we would expect cyanogenic potential to correlate positively with nodule number. We see this effect overall, without separating plants by cyanotype, as cyanogenic potential ( $\mathrm{HCNp})$ increased with increasing nodule number. However, once these data are examined within cyanotype, plants do not produce more cyanide with higher nodule numbers. Interestingly, foliar protein concentration also correlated with nodule number overall, but in the opposite direction, decreasing as plants formed more nodules, which could point to the cost of maintaining symbiosis. in a study comparing symbiotic 
plants against nitrogen-fertilized plants, plants with rhizobia had lower protein levels, along with tannins and overall biomass (Briggs, 1990). Our within-cyanotype data support this notion as HC plants, which likely have tighter allocation budgets, had quantitatively reduced protein levels as colonization intensity grew—as opposed to LC plants, in which nodule number and protein levels show a positive trend. Connecting both plant traits, neither cyanotype's HCNp responded to rhizobia colonization, but protein trends correlated with nodules in opposite directions, which may mean that $\mathrm{HC}$ plants allocate more of the total symbiotic nitrogen pool to HCNp, reducing overall soluble protein levels. Alternatively, the number of nodules $\mathrm{HC}$ plants formed may have passed a threshold from which plants quantitatively benefit from greater colonization, contributing to why plants regulate nodulation (Oka-Kira \& Kawaguchi, 2006). HC plants may have acted as a selected for relaxed autoregulation in order to attain nitrogen for constitutive cyanogenic levels, which resulted in resource allocation constraints as the cost of maintaining higher numbers of nodules may limit this additional colonization from directly benefitting leaves. In conclusion, cyanotype influences HCNp more strongly than input of nitrogen from increase nodulation, consistent with previous work, in which nitrogen treatments increased both foliar nitrogen and cyanogenesis in Eucalyptus cladocalyx, but cyanogenic levels were restricted within 
genetically-determined constraints (Simon et al., 2010). Despite nitrogen benefits, providing carbohydrates (Kaschuk et al., 2009) and specific amino acids (Prell et al., 2009) to nodules, our plant trait data demonstrate how symbiotic maintenance contributes to plant resource allocation challenges (Herms \& Mattson, 1992). Additionally, because high and low cyanotypes differentially engage in symbiosis, our data show the potential for plant defense schemes to influence the degree of symbiotic resource exchange. 

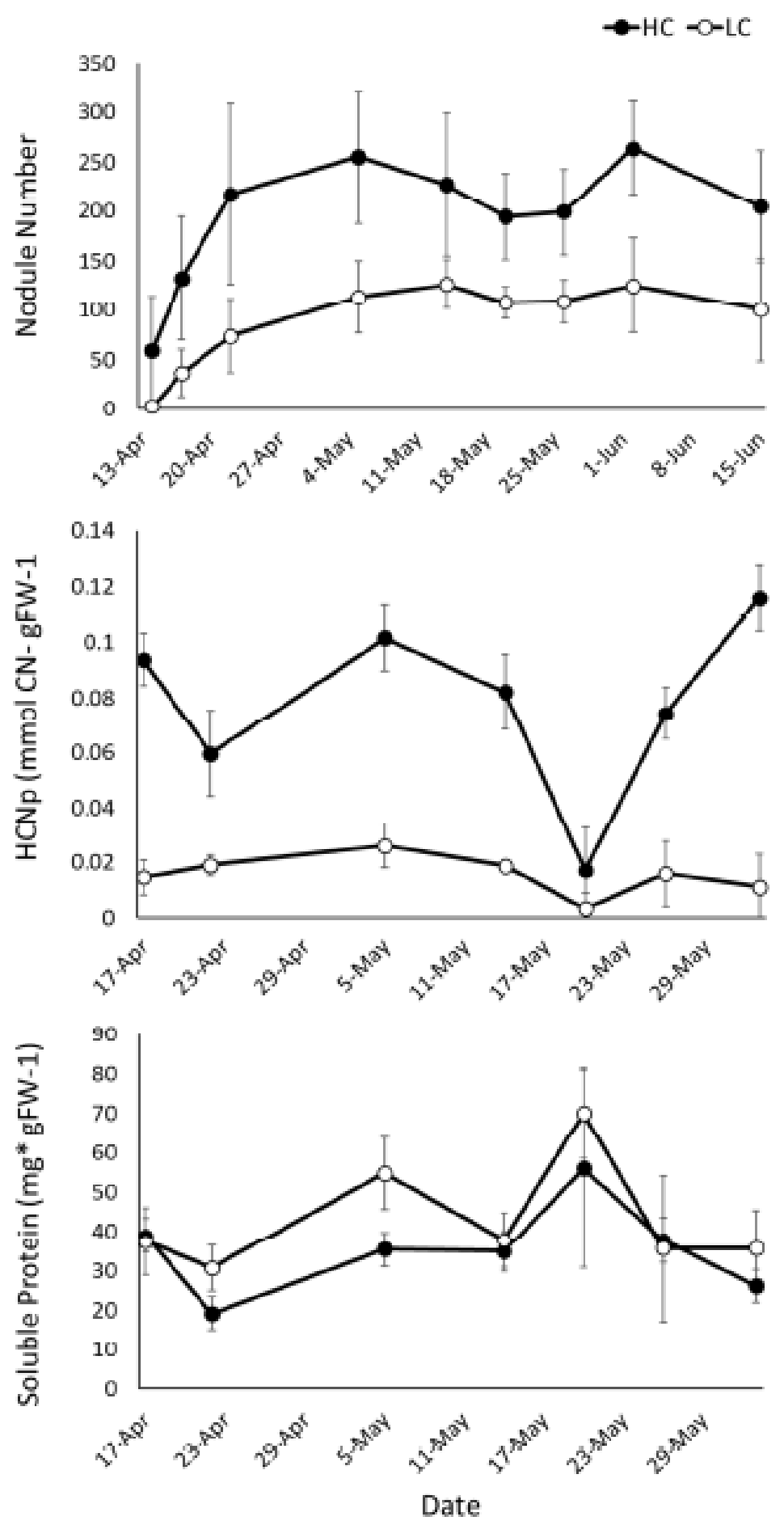

Figure 5.1 Nodulation and leaf traits differences between cyanotypes across time. Low cyanogenic plants (LC; white circles) and high cyanogenic plants (HC; black circles) measured repeatedly across a two-month span to determine A) extent of nodule colonization, B) cyanogenic potential, and C) 
soluble protein content as a nutritive trait. Points show mean values and bars represent standard deviation of the mean.
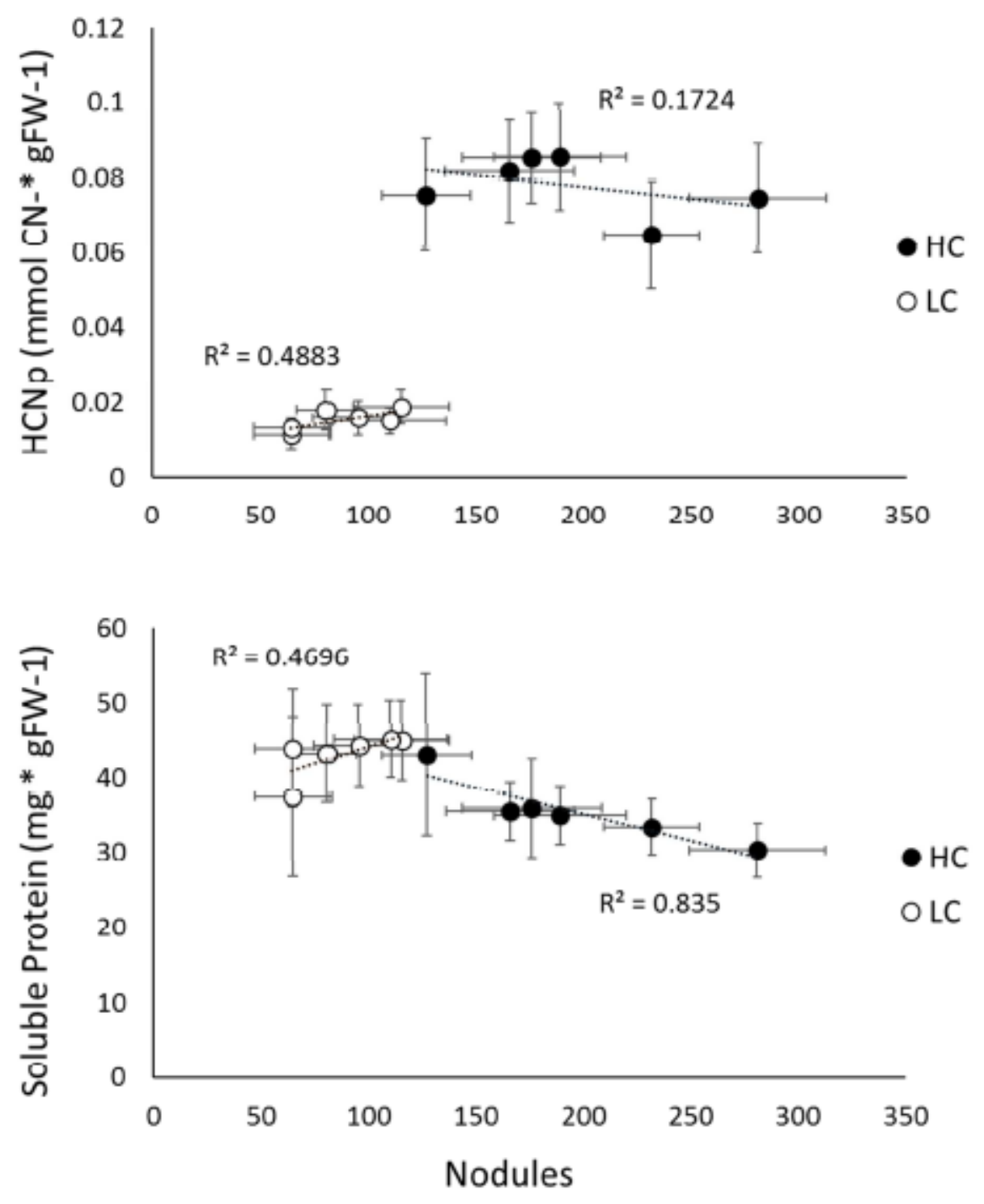

Figure 5.2. Quantitative relationships between nodule number and variation in plant traits. Plant trait values including, A) cyanogenic potential, and B) soluble protein content were averaged across the duration of the time series experiment and regressed against nodulation means to assess putative correlations. Low cyanogenic plants (LC) and high cyanogenic plants (HC) are represented by white and black circles, respectively. Points show mean 
values from repeated assays and nodule counts for each plant across the time series; bars represent standard error of the mean. 


\title{
Chapter 6: Plants with rhizobia attract fewer parasitoid wasps
}

\author{
Submitted for publication as: Godschalx AL, A Diethelm, Plants with \\ nitrogen-fixing rhizobia attract fewer parasitoid wasps. Under review \\ for publication in Journal of Insect Behavior.
}

Abstract- Predators and parasitoids influence local food webs from the topdown, and plants can benefit from indirect defense by increasing predator recruitment through chemical signaling. However, defense chemistry in plants can be affected from the bottom-up by belowground symbiotic microbes; plants extensively colonized with nitrogen-fixing rhizobia produce lower quantities overall and a unique suite of volatile organic compounds (VOCs) compared to conspecifics with less or no rhizobia. Whether the natural communities of parasitoid wasps respond to this rhizobia-mediated variation in VOC signaling has not been studied before. Here we conducted a field experiment to test for bottom-up effects of rhizobia on parasitoid wasps in nature. Using wild lima bean (Phaseolus lunatus) plants, we conducted bioassays with jasmonic acid-induced, rhizobia-inoculated $(\mathrm{R}+)$ and rhizobiafree (R-) potted plants distributed among native lima bean communities at a natural field site in Costa Rica. We quantified parasitoid recruitment for five days during three sampling periods: morning, noon, and evening. Plant biomass was not different between treatments, and showed no quantitative relationship with wasp number, yet, we found significantly greater overall 
wasp recruitment from (R-) plants, particularly in the evening. Our results indicate rhizobia symbiosis has ecologically-relevant effects on higher trophic levels in nature.

Keywords- Parasitoid wasp, volatile organic compounds (VOCs), rhizobia, symbiosis, tritrophic interactions, Phaseolus lunatus

\section{Introduction}

Damaged plants release volatile organic compounds (VOCs) that attract predators and parasitoids of herbivorous invertebrates (Turlings et al>, 1990; Thaler, 1999; Turlings \& Wäckers, 2004). Recruiting parasitoid wasps is an important aspect of plant defense, reducing herbivore damage to the plant as parasitoid larvae develop within and consume a host organism, ultimately killing the host upon emergence (Turlings et al., 1990, 1993a). By reducing plant consumer numbers, parasitoid wasps contribute to structuring the ecological community. Arthropod herbivore communities can be shaped by the abundance and diversity of parasitoids because the variation in host selection from specialist or generalist ovipositors exerts unique top-down effects on lower trophic levels (Letourneau et al., 2009). This ecosystem dynamic is particularly influenced by host-specific parasitoids (Rudgers 2004; Schmitz 2007), which recognize specific host plant chemical signals that serve as olfactory cues and influence foraging selection patterns (Turlings et al., 
1993a; De Moraes et al., 1998). Plants that can take advantage of the signalspecific responses of wasps to parasitize their herbivore enemies receive higher fitness benefits (Hoballah \& Turlings, 2001).

Attracting and retaining parasitoids can depend on plants' ability to release herbivore-induced VOCs (Thaler 1999; Röse et al. 2006). However, the emission of plant VOCs can be affected by plant-associated microbes such as rhizobia (Ballhorn et al>, 2013c). Nitrogen-fixing rhizobia in root nodules consume photosynthates from the plant in exchange for fixed atmospheric nitrogen. Plant chemical composition, including VOC chemistry, shifts due to this exchange of resources between the plant and rhizobia provisioning nitrogen while serving as a strong carbon sink (Kaschuk et al., 2009). As a result, almost all VOCs, including key compounds such as methyl jasmonate and (E)-B-caryophyllene (Thaler, 1999; Rasmann et al., 2005), were released in significantly lower amounts by rhizobia-inoculated plants relative to plants without the symbiosis (Ballhorn et al., 2013c). In contrast, specific compounds synthesized from products in the shikimate pathway, including salicylic acid and indole—a nitrogen-containing VOC, increased in plants with nitrogen-fixers (Ballhorn et al., 2013c). We designed the present study to determine whether parasitoid communities in nature respond differently to VOCs from plants with or without rhizobia. 
Predators previously have been reported to respond differently to rhizobiamediated plant traits. Rhizobia inoculation reduced secretion of extrafloral nectar, an indirect defense trait that typically recruits ant bodyguards, and consequently, fewer ants protected rhizobia-inoculated plants than rhizobiafree conspecifics (Godschalx et al>, 2015a). With evidence that predatory ants, as well as herbivores from different feeding guilds, interact with plants differently because of rhizobia-mediated chemistry (Dean et al., 2014), we hypothesized that maintaining symbiosis with rhizobia relationship would also influence the plant's interactions with parasitoid wasps. To quantify wasp recruitment, we established plant treatments with two levels of symbiosis, (rhizobia present: $\mathrm{R}+$; rhizobia absent and nitrate-supplemented: R-, distributed these plants randomly among wild lima bean communities in the field in dry tropical forest, Guanacaste, Costa Rica, and counted wasps three times daily for five days. As most studies involving responses to plant VOC production take place in controlled laboratory conditions (Turlings and Wäckers 2004), this study provides insight into the natural wasp responses faced with the effects of interacting biotic and abiotic factors inherent in natural field conditions. Specifically, here we test whether the influence of rhizobia mediating plant traits is strong enough to create differences in parasitoid recruitment under field conditions. Given that rhizobia act as a carbon sink limiting overall production of carbon-based VOC and shifting the 
volatile profiles towards nitrogen-containing VOCs such as indole, which is known to repel wasps (D'Alessandro et al., 2006), we expected to find greater wasp attraction to plants without the belowground symbiotic commitment.

\section{Methods}

Plant cultivation and field survey experiments were conducted at the Estación Experimetal Forestal Horizontes in the dry tropical forest Area de Conservación Guanacaste, Costa Rica (10.71171931, -85.57671547). Wild lima bean (Fabaceae: Phaseolus lunatus L.) plants were cultivated from seeds and allowed to germinate on a paper towel before being transplanted in 10.4 $\mathrm{cm}$ square pots filled with a 1:1 mixture of soil collected from the field station and sand collected from Playa Cabuyál, which was rinsed three times to remove salt. Both soil types were sterilized and plants were watered with sterilized water to maintain rhizobia-free status before inoculation. Plants were cultivated on a covered outdoor patio with direct morning sunlight and rotated every week to exclude position effects.

Experimental Setup_-Plants were either inoculated with rhizobia $(\mathrm{R}+$, final $\mathrm{n}=10$ ) or maintained rhizobia-free (R-, final $\mathrm{n}=7$ ) and fertilized daily during the cultivation period with a $0.25 \mathrm{mmol}^{*} \mathrm{~L}^{-1} \mathrm{CaNO}_{3}$ solution made with sterilized water, a concentration previously used to compare nitrate-fed plants to those with nitrogen-fixing rhizobia (Kiers et al>, 2006). R+ plants 
were inoculated with $50 \mathrm{~mL}$ of a water suspension of 15 homogenized nodules collected from extensively colonized wild lima bean roots at the field site. While nodule-free lima bean plants may be rare in nature due to the significant advantage from nitrogen-fixation, wild plants show strong variation in rhizobial colonization from extensive nodulation to virtually no visible nodules. Three weeks after inoculation, potted plants were moved from the outdoor cultivation site to the field, distributed within a $1 \mathrm{~m} \times 2 \mathrm{~m}$ plot among the underbrush along a roadside with direct morning sunlight that matched cultivation light conditions and contained a local population of lima bean. Plants were watered daily for an additional week before collecting parasitoid survey data.

Field Survey—Plants were sprayed with $1 \mathrm{mmol}^{*} \mathrm{~L}^{-1}$ solution of jasmonic acid to induce VOC emission every afternoon before quantifying parasitoid recruitment the following day. Parasitoids per plant were counted during 30minute sampling periods for five consecutive days, three times per day (7:30am, 12pm, and 4:30pm) to capture morning, afternoon, and evening parasitoid activity. Surveying position was rotated periodically for even visibility of all plants and all leaf surfaces. Wasps were only counted after landing on the plant surface, and approaches without contact were not included in the dataset. Individual wasps that landed twice on the same plant were only counted separately if the wasp landed on a different plant in- 
between landings. To document wasp identity, as many wasps as possible were photographed using an iPhone 6 with a Macro lens attachment (Photojojo, San Francisco, CA).

Parasitoid Community Characterization-Parasitoid wasps were identified from photos by A. Diethelm, trained and experienced in keying out parasitoid wasp specimens. Specimens were identified from photographs using wing venation patterns, shape of body, coloration, and shape of the antennae along with number of flagella (Goulet \& Huber, 1993). Wasps were identified to family level where possible following the protocol outlined by Hanson and Gauld (1995).

Plant Trait Analyses_-Following the parasitoid survey, plants were destructively harvested to quantify nodule number, above and belowground dry weight, and percentage of leaves damaged by herbivores. Roots were gently washed and nodule numbers were recorded. Both below- and aboveground plant parts were dried separately in an oven for two days and individually weighed to the nearest $0.001 \mathrm{~g}$ (NewClassic MF, Mettler Toledo). Herbivore damage was quantified (\%) as the leaf number with evidence of herbivore damage divided by the total leaf number.

Statistical Analyses_-Parasitoid response to rhizobia treatment over the course of this study was analyzed using a generalized linear model (GLM). To 
determine parasitoid recruitment differences by treatment within each time of day, two-way repeated measures ANOVAs were conducted for each sampling time and followed by Tukey's Multiple Comparison post-hoc tests. As count data with a poisson distribution, parasitoid data did not pass the Shapiro-Wilk test for normal distribution, which we intentionally did not logtransform to correct (O’Hara \& Kotze, 2010). Data followed assumptions of equal variance. Biomass data were analyzed for rhizobia treatment effects using a Welch two-sample t test. Nodule number data were utilized to generate a Pearson's regression to explain variation in percentage of leaves with damage and parasitoid visitation. Potential plant size effects on parasitoid visitation was controlled for by comparing an additional Pearson regression against shoot dry weight. Two-way repeated measures ANOVAs were conducted in SigmaPlot 12.0, and all other tests were performed in R Studio (R Core Team, 2016).

\section{Results}

Fewer parasitoid wasps were recruited to rhizobia-inoculated plants (GLM, rhizobia treatment $\mathrm{R}+$ coefficient $=-0.7133, p=0.001$, Poisson family corrected for overdispersion of residual deviance of 237 on $219 d f$; $\mathrm{AIC}=371.85$; chisquared test for goodness of fit $p=0.18)$. The greatest parasitoid recruitment took place in the evening, with 41.2 percent of all wasp visitations compared 
to 30.5 and 28.2 percent of total wasp visits during the morning and noon, respectively. Rhizobia-free plants recruited the greatest number of parasitoid wasps in the evening (Figure 1), resulting in a significant interaction between rhizobia treatment and time of day $\left(F_{12,233}=1.792, p=0.05\right)$. Separate two-way ANOVAs for morning, noon and evening revealed parasitoid recruitment was only significantly different between rhizobia treatment groups in the evening $\left(F_{1,69}=4.001, p=0.049\right)$, when the total number of parasitoid visitations to rhizobia-free plants doubled that of rhizobia-inoculated plants.

To examine quantitative relationships between rhizobia and indirect defense through parasitoid recruitment, parasitoid visits and percentage of leaves with herbivore damage were correlated with nodule number. All $\mathrm{R}+$ plants formed between 5 and 28 nodules; all but one R-plant did remain rhizobiafree, which was removed from all analyses. Increasing nodule number showed a negative trend when correlated with mean parasitoid visits, but a positive trend with percentage of damaged leaves, although neither were statistically significant (linear model based on Pearson's correlation for parasitoids: adjusted $\mathrm{R}^{2}=0.03, p=0.24$; Figure $2 \mathrm{a}$, and herbivore damage: adjusted $\mathrm{R}^{2}=0.03, p=0.25$; Figure $2 \mathrm{~b}$.)

To explain differences in parasitoid recruitment between rhizobia treatments $\mathrm{R}+$ and $\mathrm{R}$-, we tested for differences in plant size as larger plants may attract 
more parasitoids. Plant biomass, both below- and aboveground dry weight were not significantly different by treatment $(t=-0.59, p=0.57$, and $t=-1.22$, $p=0.24$, Welch two-sample t-test, $d f=13$, for below and aboveground dry weight, respectively; Figure 3). Quantitatively, aboveground biomass did not explain variation in mean parasitoid visitation per plant (adjusted $R^{2}=-0.07$, $p=0.87$; Figure 4c), and did not form a significant relationship with nodule number despite a positive trendline (adjusted $\mathrm{R}^{2}=-0.07, p=0.87$; Figure $4 \mathrm{~d}$ ). Root biomass was significantly influenced positively by nodule number (adjusted $\mathrm{R}^{2}=0.28, p=0.02$; Figure $4 \mathrm{~b}$ ), which in turn showed a negative trend with parasitoid visits, although this correlation between root dry weight and mean recruitment was not significant (adjusted $\mathrm{R}^{2}=0.04, p=0.20$; Figure $4 \mathrm{a}$ ).

Among all visitations, parasitoids recruited to experimental plants in the field included 22 morphologically distinct parasitoid wasps. Five of those parasitoids were from the superfamily Chalcidoidea, including two belonging to the family Aphelinidae. Four of the morphologically distinct parasitoids belonged to the Ichneumonidea superfamily, with at least one parasitoid from the Braconidae family and one parasitoid from the Ichneumonidae family.

\section{Discussion}

Both rhizobia and parasitoid wasps are key plant mutualists in many terrestrial ecosystems. Parasitoid wasps collectively utilize a wide range of 
insect herbivores as food sources for their larvae, and thus, play an important role in plant defense and community ecology. Rhizobia as microbial mutualists directly promote growth of legume host plants by providing nitrogen in exchange for photosynthates (Kaschuk et al>, 2009), which simultaneously alters plant chemistry with a cascading effects on plantherbivore (Dean et al., 2009; Thamer et al., 2011; Ballhorn et al., 2013c) and plant-carnivore interactions (Godschalx et al., 2015a). Although plantparasitoid and plant-rhizobia interactions are generally well-studied, studies on the interaction of all three partners are scarce (Pineda et al., 2013; Pangesti et al., 2015a)—particularly under natural conditions. Here we report a reduced parasitoid presence on wild lima bean plants with symbiotic, nitrogen-fixing rhizobia compared to nodule-free conspecifics in nature.

The observed higher parasitoid attraction towards rhizobia-free plants compared to rhizobia-inoculated plants may include a combination of repellent and attractive effects resulting from i) rhizobia acting as a carbohydrate sink, reducing plant volatile signal strength and ii) symbiontaltered plant chemical pathways. Plant volatile chemistry is the direct interface between plant and parasitoid wasp (Turlings et al>, 1990; Thaler, 1999) and has been shown to depend on rhizobial symbiosis, both with nodule-forming rhizobia and soil rhizobacteria (Pineda et al., 2013; Ballhorn et al., 2013c; Pangesti et al., 2015b). Like other carbohydrate-demanding 
microbes including mycorrhizal fungi, nodulating rhizobia act as a strong carbon sink (Kaschuk et al., 2009; Pringle, 2015b) and can lead plants to produce lower quantities of volatiles overall (Fontana et al., 2009; Ballhorn et al., 2013c). In addition to reducing total signal strength, rhizobia reduce levels of specific volatiles demonstrated to specifically attract parasitoids: jasmonic acid (JA)-induced octadecanoid products (Thaler, 1999). As an antiherbivore plant hormonal pathway, JA-inducible defenses likely provide reliable cues from plants suffering from herbivory to parasitoids indicating the presence of a future food source. Parasitoids showed no preference among plants with or without rhizobacteria from aphid-infested Arabidopsis plants genetically modified to inhibit JA production, whereas wild-type plants recruited fewer parasitoids when rhizobacteria were present (Pineda et al., 2013), consistent with our field data. Our data showing fewer parasitoids were attracted to plants with rhizobia could be an example of rhizobia directly reducing a plants' ability to produce the JA-inducible volatile products most important for attracting parasitoids as an indirect defense. Quantitatively, we observed trends that would support this idea, with a positive, although not significant, relationship between nodule number and percentage of leaves showing herbivore damage, coupled with a trend suggesting an increase in nodules may reduce parasitoid attraction, although further work is needed to establish a quantitative carbon sink is the 
interfering factor reducing indirect defense in this case.

Alternatively, parasitoids may be repelled by particular volatiles produced by plants with rhizobia. Nodule formation is technically a microbial infection, and plants primed for pathogen resistance form fewer nodules (Ballhorn et al>, 2014c), indicating an interaction between plant-microbe symbiosis and defense pathways. Nodulation could therefore cause an increase in antipathogen defenses induced by salicylic acid, as well as volatiles known to interact with other plant-associated organisms, including indole. Indole and salicylic acid were the only measured VOCs to increase in rhizobia-inoculated wild lima bean plants in a previous study (Ballhorn et al., 2013c), leading us to design the present study to test whether parasitoids would respond to plant tissue likely releasing more indole. Indole primes plants to release volatiles in response to damage and herbivore-specific elicitors (Erb et al., 2015), but the increase in indole from rhizobia-influenced plants may also directly impact parasitoids due to the general toxicity of this compound. Evidence for indole toxicity comes from herbivores raised on indole-producing food which - although the herbivores grew bigger - they also had higher mortality and preferred food without indole despite restricted growth (Veyrat et al., 2016). If soybean plants with rhizobia also produce higher levels of indole, this could also be the case for soybean podworms (Helicoverpa zea), which avoid but have increased growth on plants nutritionally relying on 
rhizobia (Dean et al., 2014). In our lima bean system, rhizobia-inoculated lima bean VOCs and rhizobia-free lima bean VOCs with added synthetic indole at natural concentrations equally repelled specialist Mexican bean beetles (Ballhorn et al., 2013c), which further supports the antagonistic effect of indole on insects. As various herbivores are negatively affected by indole and respond accordingly to rhizobia-mediated plant chemistry, our results may not be surprising that parasitoids also prefer rhizobia-free plants. In fact, a study by D'Alessandro and co-workers (2006) showed that some parasitoids specifically avoid indole—-both from natural and synthetic sources.

Whether or not it is an adaptive response for parasitoids to avoid indoleemitting or rhizobia-inoculated plants is not known. Parasitoid larvae developed more rapidly and with a greater resulting mass on plants with arbuscular mycorrhizal fungi (Hempel et al>, 2009). In another example but without microbes, nutritional status influenced parasitoid success as nitrogen-treated Glycine max-fed herbivores increased parasitoid larval mass, and quantitatively altered VOC emission, but adult wasps did not prefer these plants over nitrogen-deficient plants. (Winter \& Rostás, 2010). To address specific plant-rhizobial effects on herbivores as a food source for parasitoids, an interesting follow-up study would be to test whether or not herbivores fed on rhizobia-free plants are a better food source for developing 
wasps compared with herbivores consuming rhizobia-inoculated plant tissue.

If parasitoids are deterred by rhizobia-induced chemistry, it remains unclear whether this choice is in response to the reduced likelihood of finding herbivores on rhizobial plants, or if rhizobia-driven volatiles provide cues about additional plant trait information that may impact parasitoid survival. Multiple plant defense traits co-vary with volatile production (Ballhorn et al>, 2008b, 2013a), including extrafloral nectar (EFN), which can be an important source of water and sugars for parasitoids (Limburg \& Rosenheim, 2001), particularly in dry habitats such as the Costa Rican tropical dry forest where we conducted this study. EFN is produced in lower quantities in rhizobial plants (Summers \& Mondor, 2011; Godschalx et al., 2015a), along with total VOC expression. Both indirect defense traits trade off with a direct chemical defense, cyanogenesis, which is generally expressed at high levels in wild lima bean plants with rhizobia (Thamer et al., 2011). If cyanide exposure reduces food quality for parasitoid larvae by negatively impacting herbivores (Ballhorn et al., 2007), the volatiles expressed in high quantities from less cyanogenic plants could guide parasitoid host selection, although this would need empirical support to confirm.

Interestingly, parasitoids significantly preferred rhizobia-free plants during the evening sampling time. Herbivore-induced plant volatiles follow a 
photoperiod-dependent circadian rhythm with peaks occurring late afternoon (Christensen et al>, 2013). If volatile expression peaks coincide with parasitoid activity, this may explain why most of the wasp recruitment took place during our 4:30pm sampling time. Additionally, we treated plants with JA for VOC induction, which is also connected to light cycles as isoleucine-JA biosynthesis and extrafloral nectar secretion are light dependent (Radhika et al., 2010b). Although we did not measure VOCs in this experiment, photoperiod-dependent biosynthesis supports that the increased wasp activity we observed in the evening was likely related to an increase in VOC emission.

Parasitoid activity is also likely connected to circadian rhythms of herbivorous hosts. Due to the phylogenetically diverse group of parasitoids observed in our study, the specific herbivores each parasitoid utilizes as a host likely spans a diverse spread of feeding guilds. We observed a diversity of parasitoid wasps, including wasps from both the Chalcidoidea and Ichneumonoidea superfamilies, belonging to the Aphelinidae, Ichneumonidae and Braconidae families. Ranging from specialist to generalist predators, parasitoid activity may largely depend on oviposition patterns and active feeding times of Homoptera, Coleoptera, Lepidoptera, and other herbivorous hosts. Chewing insects damage leaf tissue to a greater extent than phloem feeders, which alone can influence plant VOC emission differences, but 
additionally, feeding mode can influence plant hormonal regulation (Bonaventure, 2012) as well as subsequent volatile chemistry (Tan \& Liu, 2014), These different effects help explain why herbivore identity matters in understanding microbially-mediated parasitoid attraction. Our data showing fewer parasitoids on plants with rhizobia is consistent with soil rhizobacteriaassociated plants infested with aphids (Pineda et al>, 2013) but not leafchewing insects (Pangesti et al., 2015b). Future work is needed to characterize which specialist parasitoids are attracted towards or repelled from plants with symbiotic rhizobia, which would be an interesting visualization of the bottom-up trophic level effects of rhizobia with greater feeding guild resolution. Without information about taxonomic identity of wasps on either rhizobia treatment $(\mathrm{R}-, \mathrm{R}+)$, it is interesting that we observed parasitoids across a relatively broad range of taxa responding relatively consistently to plants in natural communities depending on rhizobia symbiosis. Our results indicate that aboveground signaling mediated by belowground symbiosis can impact the third trophic level in nature. Therefore, symbiotic, nitrogen-fixing rhizobia contribute to an integrated ecological system in which bottom-up forces affecting the recruitment of parasitoids subsequently influence top-down forces via plant defense. 


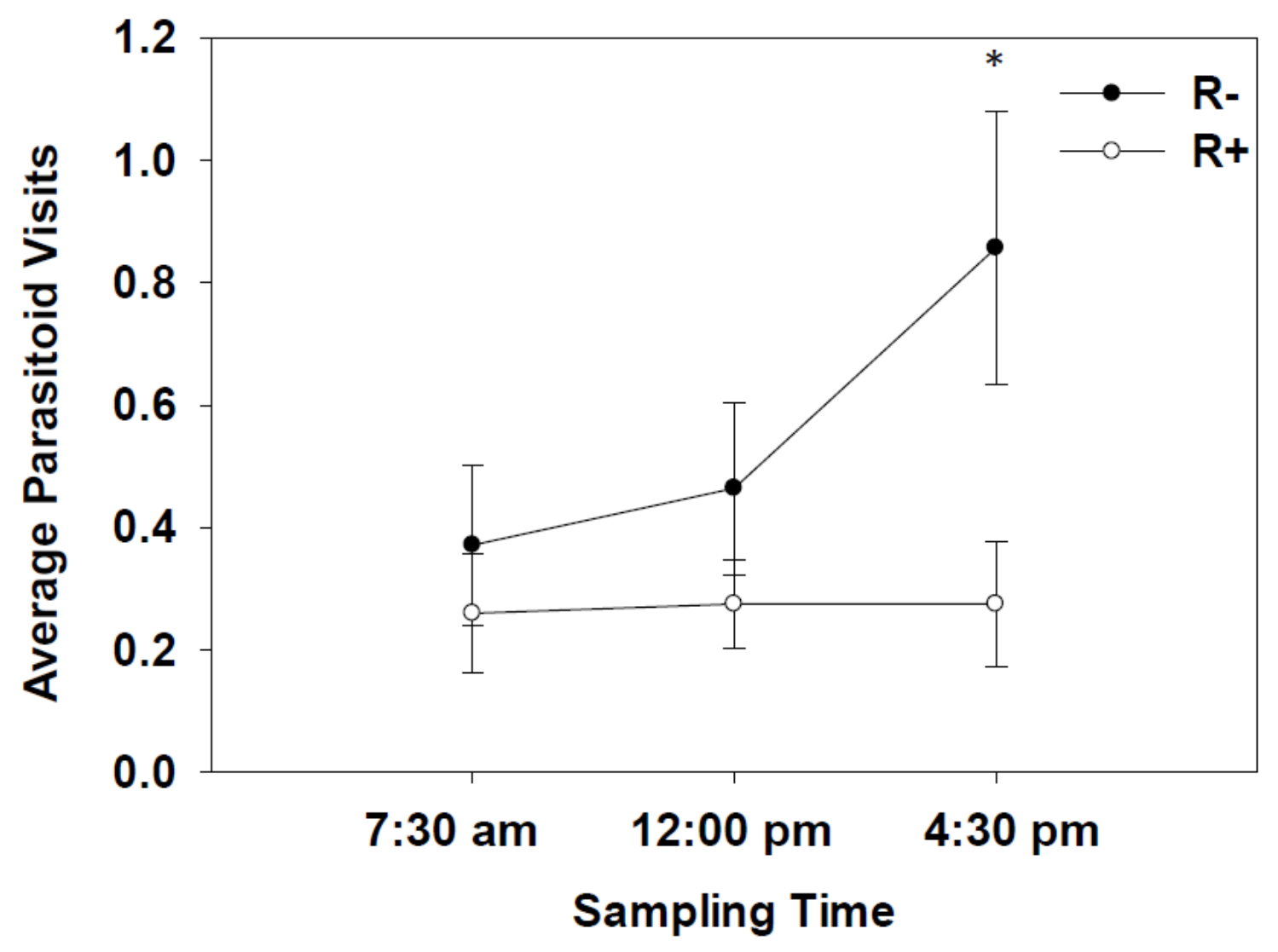

Figure 6.1 Parasitoid wasps preferred rhizobia-free plants over rhizobiainoculated plants among natural community. Points indicate the mean number of parasitoids counted per plant averaged across all five sampling days for each of the three sampling time points: 7:30am, 12pm, and 4:30pm. Error bars show standard error of the mean. White circles represent rhizobiainoculated plants, $n=10$, and black circles represent rhizobia-free plants supplemented with nitrate, $n=7$. Significant differences in parasitoid count averages between rhizobia treatments for each sampling time from separate one-way ANOVAs is indicated with *, indicating $\mathrm{p}<0.05$. 

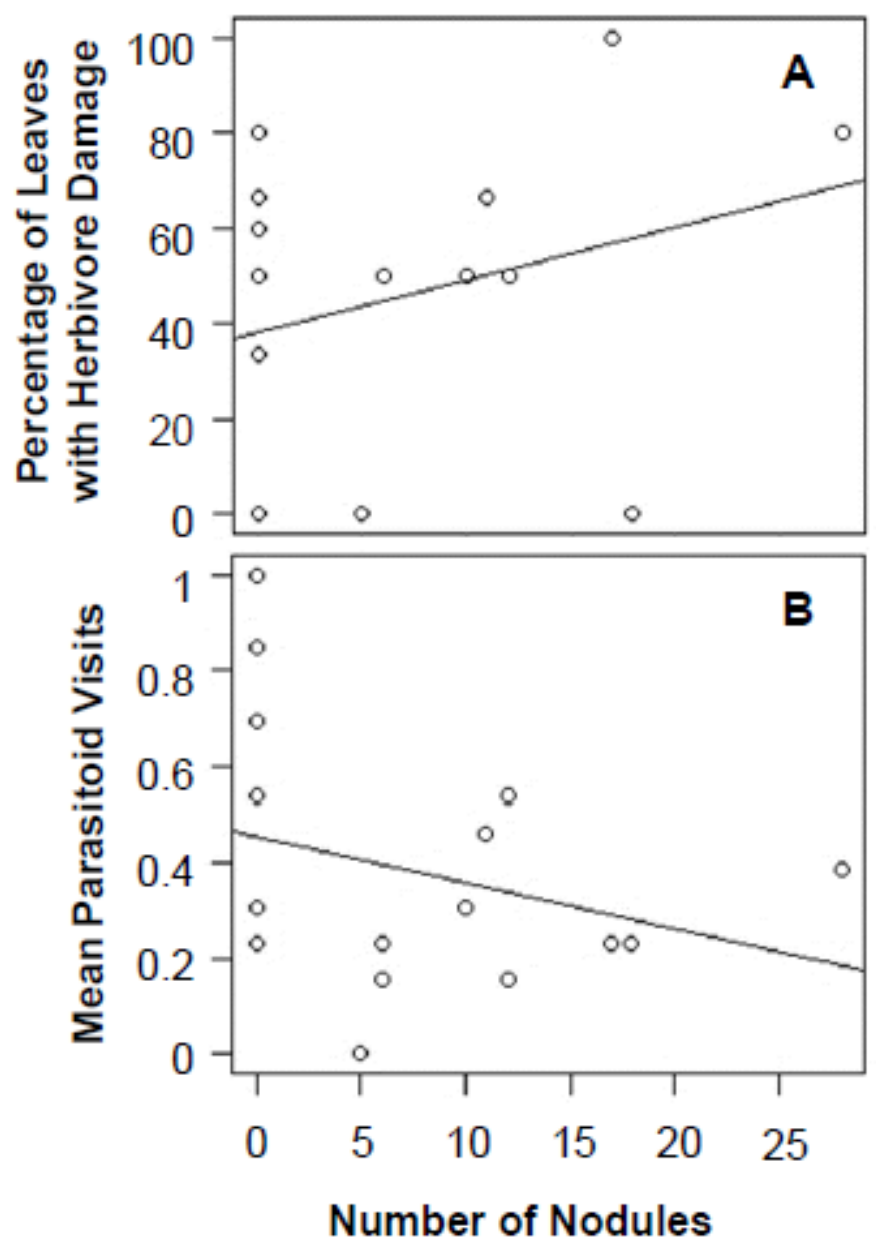

Figure 6.2 Nodule number quantitative relationships with leaf damage and parasitoid visitation. Points indicate values for each plant replicate $(n=17)$, with mean nodule number regressed against $A$ ) the percentage of leaves with herbivore damage, and B) the mean number of parasitoids per plant averaged across all sampling time points across five sampling days. Trendlines based on linear models with Pearson's regression, $\mathrm{p}=0.25$, and $\mathrm{p}=0.24$ for $\mathrm{A}$ and $\mathrm{B}$, respectively. 


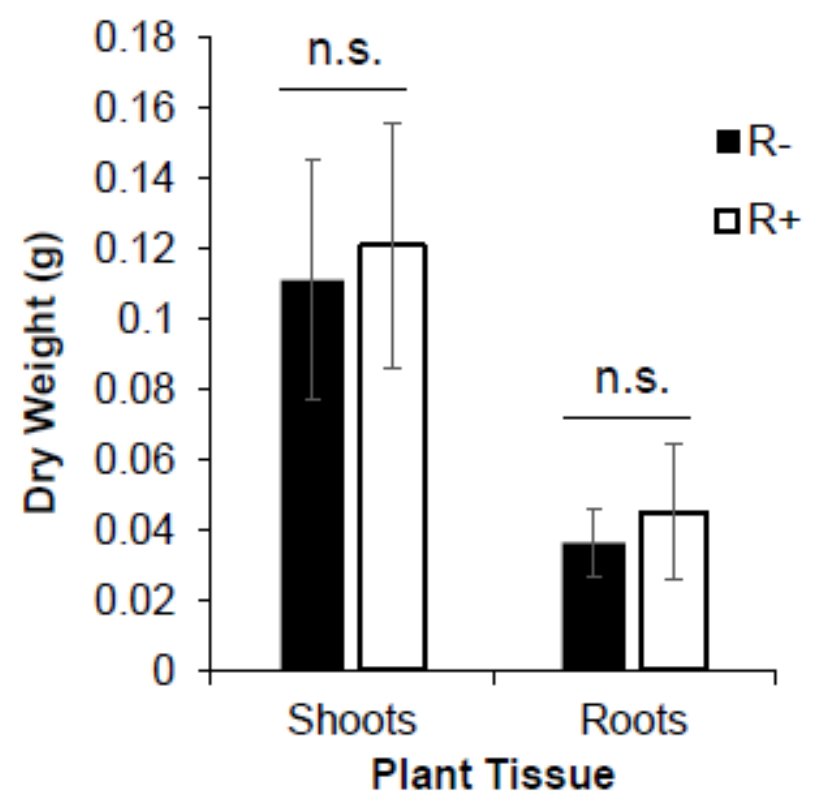

Figure 6.3 Plant below- and aboveground biomass did not vary by rhizobia treatment. Bars indicate mean dry weight values for entire plant shoot and root systems for plants inoculated with rhizobia (white bars, $\mathrm{n}=10$ ) or rhizobia-free plants supplemented with nitrate (black bars, $n=7$ ). Error bars show standard deviation of the mean. Significance values from Welch twosample $t$ tests indicated by n.s. represent $p=0.57$ for shoot tissue, and $p=0.24$ for root tissue. 
Roots

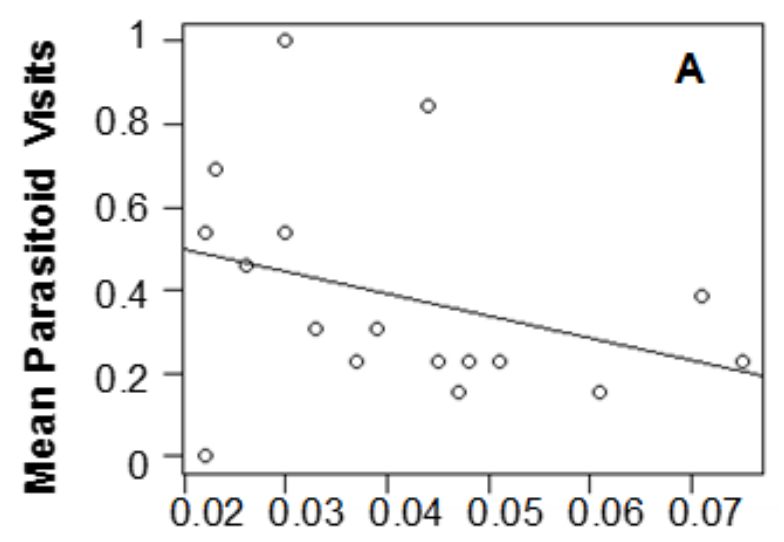

Shoots

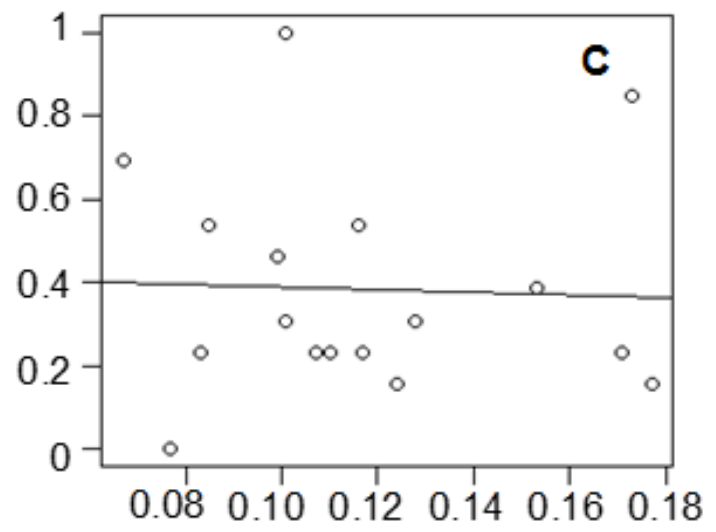

Dry Weight (g)
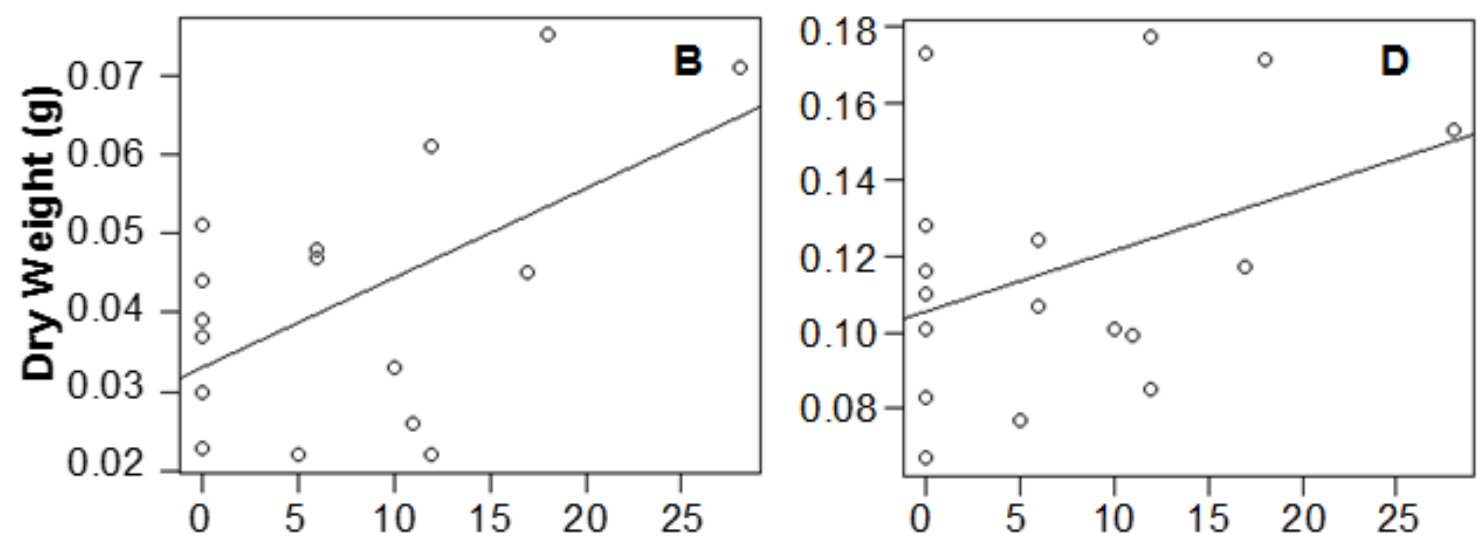

Number of Nodules

Figure 6.4 Plant above- and belowground biomass quantitative relationships to parasitoid recruitment. Points indicate values for each plant replicate $(\mathrm{n}=17)$, with the mean number of parasitoids per plant averaged across five sampling days regressed against dry weight for A) roots, and C) shoots. Final dry weight values regressed against number of nodules shown for B) roots and D) shoots. Trendlines based on linear models with Pearson's regression returned significance values of $\mathrm{p}=0.20, \mathrm{p}=0.02^{*}, \mathrm{p}=0.87$ and $\mathrm{p}=0.19$ for $\mathrm{A}, \mathrm{B}, \mathrm{C}$ and $\mathrm{D}$, respectively. 


\section{Chapter 7: Plants with rhizobia attract fewer ants}

\section{Published as: Godschalx AL, Schädler M, Trisel JA, Balkan MA,}

Ballhorn DJ. 2015. Ants are less attracted to the extrafloral nectar of plants with symbiotic, nitrogen-fixing rhizobia. Ecology 96: 348-354.

Abstract: Plants simultaneously maintain mutualistic relationships with different partners that are connected through the same host, but do not interact directly. One or more participating mutualists may alter their host's phenotype, resulting in a shift in the host's ecological interactions with all other mutualists involved. Understanding the functional interplay of mutualists associated with the same host remains an important challenge in biology. Here we show belowground nitrogen-fixing rhizobia on lima bean (Phaseolus lunatus) alter their host plant's defensive mutualism with aboveground ants. We induced extrafloral nectar (EFN), an indirect defense acting through ant attraction, measured various nutritive and defensive plant traits, biomass, and counted ants on rhizobial and rhizobia-free plants. Rhizobia increased plant protein and cyanogenesis, a direct chemical defense against herbivores, but decreased EFN. Ants were significantly more attracted to rhizobia-free plants, and our structural equation model shows a strong link between rhizobia and reduced EFN as well as between EFN and ants: the sole path to ant recruitment. The rhizobia-mediated effects on simultaneously expressed defensive plant traits indicate rhizobia can have significant bottom-up effects on higher trophic levels. Our results show 
belowground symbionts play a critical and underestimated role in determining aboveground mutualistic interactions.

Key Words Extrafloral nectar, plant defense, rhizobia, ant-plant, multitrophic interactions, cyanogenesis, direct defense, indirect defense, lima bean, plant resource allocation, mutualism, nitrogen fixation

Introduction: Mutualistic relationships with microbes such as rhizobia, which fix atmospheric nitrogen $\left(\mathrm{N}_{2}\right)$, relieve the nitrogen limitation many plants experience in natural ecosystems. By making this otherwise inaccessible nitrogen available to their plant host, rhizobia are widely considered ecosystem engineers and keystone species: having a disproportionate impact on other organisms including both plant antagonists and mutualists (Crooks, 2002). For example, nitrogen-fixation in root nodules critically impacts plant fitness, significantly altering resources available for plants to grow and defend themselves against herbivores by enhancing direct chemical defenses (Dean et al., 2009; Kempel et al., 2009b; Thamer et al., 2011) or altering the indirect defenses plants use to recruit mutualistic invertebrates (Ballhorn et al., 2013c). Despite extensive knowledge on the benefits of rhizobia on plant growth-related parameters, the impacts of rhizobia on these diverse aboveground plant-insect interactions have only recently been explored. Our aim for the current study is to understand how 
rhizobia alter plant extrafloral nectar (EFN, nectar not involved in pollination), serving as a plant indirect defense by attracting mutualist predatory ants.

EFN represents one of the most widely distributed indirect defenses in the plant kingdom, including angiosperms, gymnosperms, and ferns (Weber \& Keeler, 2013). EFN acts as a defense by attracting carnivorous arthropods, particularly ants—one of the most dominant insect groups in terrestrial ecosystems. Ants play a crucial role as defenders by attacking or evicting invertebrate herbivores from the plant, therefore, $\mathrm{EFN}$ is considered an effective indirect defense (McLain 1983, Ness et al. 2009). The plant kingdom contains a morphologically diverse suite of extrafloral nectaries with varying types of EFN secretion. Obligate ant-plants constitutively produce EFN along with lipid-rich food bodies to nourish defending ants (Wagner, 1997), whereas facultative ant-defended plants express EFN as an inducible defense only when damaged, conserving resources until the plant is under attack (Kost \& Heil, 2008). Lima bean (Phaseolus lunatus), the experimental plant used in this study, represents a facultative ant-plant which increases the production of EFN when damaged. EFN is generally comprised of sugars derived from photosynthesis and therefore has historically been considered a metabolically ‘cheap' defense. However, some EFN contain a subset of amino acids to supplement the ants' diet as well as specific defensive components such as 
alkaloids and tannins to deter nectar-robbers and to exclude microorganisms, thereby increasing the plant's metabolic costs in nectar production (Heil, 2011). EFN secretion is also dependent on soil nutrients and available photosynthetic leaf area, further indicating that EFN production comes at a cost to the plant (Mondor et al. 2006, Li et al. 2012, Ballhorn et al. 2014). Metabolic costs of EFN would imply secretion could be limited in plants with an additional carbon sink, such as rhizobial symbiosis.

The effect of rhizobia on EFN production has not been studied before. Lima bean is a particularly interesting system to use in understanding plant defense because this model organism expresses multiple defenses in concert with EFN (Ballhorn et al., 2008b). Lima bean releases volatile organic compounds (VOCs), which indirectly defend plant tissue by attracting parasitoid wasps that oviposit in the plant's enemies (Kost \& Heil, 2008; Ballhorn et al., 2008b). Furthermore, lima bean shows various direct chemical defenses, including cyanogenesis, the wound-induced release of hydrogen cyanide from preformed precursors, as well as polyphenol oxidases effective against herbivores and fungal pathogens (Ballhorn et al., 2010b).

Evidence for defense syndromes and tradeoffs between suites of traits is well documented in lima bean (Ballhorn et al., 2013a), making this system particularly suitable to examine the effects of rhizobia mutualism on plant 
traits, as well as the impacts on higher trophic levels. In this study, we experimentally manipulated rhizobial colonization to measure effects of the microbial symbionts on ant recruitment to the plant hosts' extrafloral nectar. If the carbon costs of rhizobia symbiosis and EFN conflict, we expected a rhizobia-mediated decrease in EFN production, with a cascading influence on ant attraction.

Materials and Methods: Study species: Wild lima bean (Fabaceae:

Phaseolus lunatus L.) plants were grown from seeds collected in Mexico $\left(15^{\circ} 55^{\prime} 31.80^{\prime \prime} \mathrm{N}, 97^{\circ} 9^{\prime} 4.68^{\prime \prime} \mathrm{W}, 8 \mathrm{~m}\right.$ a.s.l.). Plants were grown in containers (one plant per pot) $12 \mathrm{~cm}$ in diameter in greenhouse mix \#3 (SunGro Horticulture ${ }^{\circledR}$, Bellevue, WA), 175g per pot. Potting soil was washed thoroughly three times to reduce substrate nutrients. Seeds with a nicked seed coat germinated on moist paper towel and were subsequently planted $0.5 \mathrm{~cm}$ below soil surface. Plants were watered daily and moved monthly to avoid microclimatic effects. Plants were potted and cultivated at Portland State University from July $7^{\text {th }}$-October $4^{\text {th }}, 2013$ on the outdoor cultivation area of the research greenhouse to allow sun exposure. Plants were not protected from ambient herbivory in order to measure plant defense responses that more closely resemble plants in natural sites.

Experimental setup: Rhizobia inoculum was prepared by grinding three 
nodules for a total of 0.1g fresh weight (Accession DJB1033, Ballhorn Lab) suspended in 1L water (Ballhorn et al. 2013a). Once seedlings had two leaves, $100 \mathrm{~mL}$ rhizobia inoculum $\left(>10^{7}\right.$ cells $\left.\mathrm{mL}^{-1}\right)$ was poured on the soil at the base of each seedling representing $\mathrm{R}+$ (with rhizobia) treatment plants $(\mathrm{n}=33) ; 100 \mathrm{~mL}$ sterile water was poured on the soil of each seedling representing $\mathrm{R}$ - (without rhizobia) treatment plants $(\mathrm{n}=20)$. Two weeks after inoculation, all $\mathrm{R}+$ plants and two of the R-plants showed signs of early nodulation. At the end of experiments when plants were destructively harvested, these same plants had uniformly high nodulation, so the data for these two plants were added to the $\mathrm{R}+$ treatment for a final sample size of R-, $\mathrm{n}=18$ and $\mathrm{R}+, \mathrm{n}=35$.

Plant trait analyses: We selected young, intermediate and mature leaves for all plant biochemical analyses to account for ontogenetic variability of plant traits. Young leaves were defined as the uppermost unfolded leaf on the vine, intermediate leaves were chosen two leaves below young leaves, and mature leaves were selected two leaves below the intermediate leaves. Extrafloral nectar (EFN) was quantified according to Kost and Heil (2006). To induce indirect defense, jasmonic acid (JA; $1 \mathrm{mmol} / \mathrm{L})$ was applied to foliage and $24 \mathrm{~h}$ later, young, intermediate and mature leaves were mechanically damaged with a toothbrush. Twelve hours after mechanical damage, EFN volume was collected with microcapillaries (PCR Micropipets 1-10 $\mu$ l; Drummond). Sugar 
concentration in EFN was quantified with a Brix 0-18\% refractometer (Fisher Scientific). Leaves used for EFN collection were harvested and dried (IncuMax CV250 Convection Oven, Amerex Instruments Inc.) at $72^{\circ} \mathrm{C}$ for 9 days. EFN values were calculated as mg sugar/g leaf dry weight. The cyanogenic potential (HCNp; concentration of cyanogenic precursors) of leaves of similar developmental stages (next to leaves collected for EFN) was quantified using the Spectroquant ${ }^{\circledR}$ cyanide test (Merck, Darmstadt, Germany) (Ballhorn et al., 2008b). In short, leaves were removed, weighed to the nearest $0.001 \mathrm{~g}$ (NewClassic MF, Mettler Toledo) and three leaf punches were ground with a mortar and pestle at $4^{\circ} \mathrm{C}$ in two $\mathrm{mL}$ ice-cold $\mathrm{Na}_{2} \mathrm{HPO}_{4}$ buffer (67 mmol/L). Samples were analyzed for HCNp by complete enzymatic hydrolysis of cyanogenic precursors in gas-tight glass vessels (Thunberg vessels). Spectrophotometric quantitative detection of HCN was carried out at $585 \mathrm{~nm}$ Plant nutritive quality as total soluble protein (from the same leaf samples taken for $\mathrm{HCN}$ ), was measured spectrophotometrically at $595 \mathrm{~nm}$ using the Bradford protein assay (Ballhorn et al. 2011; Ganzhorn 1992). A calibration curve ranging from $50 \mu \mathrm{g} / \mathrm{mg}$ to $1000 \mathrm{\mu g} / \mathrm{mg}$ was prepared with bovine serum albumin (Amresco). Total biomass was measured for above- and belowground fresh weight and dry weight. None of the plants flowered or set fruit.

Ant recruitment: To quantify ant attraction, induced and control plants were 
randomly distributed $1.5 \mathrm{~m}$ apart on a gravel area near the research greenhouse facility at Portland State University. Four dead twigs connected the soil with the foliage of each plant to allow exposure to naturally occurring pavement ants (Tetramorium caespitum), which were observed frequently throughout the gravel strip. Ants per plant were counted 8h after EFN induction.

Statistical analyses: We used linear models (type III sum of squares) to analyze the effects of rhizobia on plant biomass. Effects of rhizobia on leaf traits were analyzed using a split-plot linear mixed model with plant individual as random main plot-factor and leaf age as sub plot-factor within plant individual. Number of ants was analyzed as a generalized linear model (GLM), assuming poisson-distributed residuals with the loglink function (all analyses in SAS 9.2, Proc GLIMMIX). Tukey's HSD-test was used for posthoc comparisons of means. To examine the strength of indirect and direct interactions between rhizobia, plant traits, and ants, structural equation modeling was used (SPSS AMOS 22), which allowed us to make conclusions about the relationship between all measured variables in a single analysis (Grace, 2006). Rhizobia treatment was used as a binary exogenous variable; aboveground plant traits and number of ants were used as observed endogenous variables. For our initial conceptual model we assumed that rhizobia affected all aboveground plant traits but had no direct effect on ants. 
We assumed possible effects of all leaf traits on ant abundance since not only extrafloral nectar but also leaf protein and $\mathrm{HCN}$ could be suggested to influence ant attraction. However, we did not assume an effect of shoot biomass on ant abundance (all plants grew in pots of the same size, therefore larger plants did not represent larger targets). We further assumed possible covariation between all aboveground plant traits. Adequate model fits were confirmed by non-significant $X^{2}$-tests $(P>0.05)$ and low RMSEA $(<0.05)$. Stepwise removal of unimportant relationships was used to optimize the model based on these model fit indices (see Appendix B for the initial conceptual model and the model selection procedure). Data met the assumptions of SEM and did not deviate from normality and included no statistical outliers (SPPS AMOS 22). Bivariate relationships between response variables are given in Appendix D.

Results: Rhizobia-inoculated plants recruited fewer ants. Three times more ants were found on rhizobia-free plants (R-) as compared to plants with rhizobia symbiosis $(\mathrm{R}+)$ (Fig. 1, GLM: $\left.F_{1,51}=19.10, P<0.001\right)$. We deciphered the net effect on ant recruitment in a structural equation model (SEM) explaining $45 \%$ of the variation in ant attraction. The sole significant path to ant abundance was mediated through extrafloral nectar (EFN), which ultimately was determined by rhizobia treatment (Fig. 2). Rhizobia directly reduced EFN with a path coefficient of -0.66, and this final model explained 
$44 \%$ of the variation in EFN. EFN did not significantly interact with any other plant trait. The direct link between rhizobia and EFN was not mediated by changes in biomass. Shoot biomass had a significant positive interaction with leaf protein and cyanogenic potential (HCNp). Rhizobia had strong direct positive effects on shoot biomass, protein, and HCNp with path coefficients of $0.74,0.62$, and 0.60 , respectively (Fig. 2). Protein and HCNp did not affect ant recruitment as the standardized estimates were not significant $(P<0.12)$. Analysis of variance results for individual plant traits confirmed the same relationships described in the SEM for the entire plant system with rhizobia treatments. Rhizobia symbiosis significantly affected all measured plant traits. $\mathrm{R}+$ plants produced more aboveground and belowground biomass (Appendix A, all $P<0.001$ ). R- plants had a shoot average of $2.29 \pm 0.23 \mathrm{~g} \mathrm{dw}($ mean $\pm \mathrm{SE})$, whereas shoot biomass increased more than threefold in $\mathrm{R}+$ plants at $9.77 \pm 0.70 \mathrm{~g}$. Root biomass increased more than twofold in $\mathrm{R}+$ plants $(3.4 \pm 0.33 \mathrm{~g} \mathrm{dw})$ relative to $\mathrm{R}$ - plants $(1.45 \pm$ $0.11 \mathrm{~g} \mathrm{dw}$ ). Rhizobia significantly increased leaf protein concentration (Fig. $\left.3 \mathrm{~A}, F_{1,51}=31.16, P<0.001\right)$, with the greatest difference between rhizobia treatments being in young leaves $\left(F_{2,102}=24.96, P<0.001\right)$, but the interacting effects of rhizobia treatment and leaf age were not significant $\left(F_{2,102}=2.15, P=0.12\right)$. Cyanogenic potential $(\mathrm{HCNp})$ followed a similar pattern to that of protein concentration; rhizobia increased cyanogenesis (Fig. 
3B, $\left.F_{1,51}=29.13, P<0.001\right)$, and this chemical defense decreased as leaves aged $\left(F_{2,102}=170.04, P<0.001\right)$. However, unlike protein content, HCNp was affected by the interacting effects of rhizobia and leaf age, with the younger, more productive leaf tissues more highly defended $\left(F_{2,102}=8.73, P<0.001\right)$.

Extrafloral nectar (EFN) secretion was highest in young leaves of R-plants (Fig. 3C). Rhizobia reduced EFN $\left(F_{1,51}=39.43, P<0.001\right)$, and increasing leaf age lowered $\operatorname{EFN}\left(F_{2,102}=26.01, P<0.001\right)$. The interacting effects of rhizobia and leaf age was significant $\left(F_{2,102}=16.21, P<0.001\right)$; $\mathrm{EFN}$ values were almost four times higher for R-young leaves compared to young leaves on R+ plants (Fig. 3C). In summary, young, actively growing leaves on plants with rhizobia produce less extrafloral nectar than young leaves on plants without nitrogenfixing symbionts, mediating effects on the aboveground ant-plant symbiosis.

Discussion: Rhizobia are recognized keystone mutualists, yet the impacts rhizobia have on aboveground interspecific interactions, particularly on mutualistic relationships, are still gradually being revealed. In this case, the rhizobia-legume mutualism and the ant-plant mutualism are in conflict in the same host plant system. Both of these mutualisms are well studied and often serve as a model for understanding the evolutionary implications of mutualistic relationships (Kiers et al., 2003; Mayer et al., 2014). Yet, surprisingly, the effects of both mutualisms interacting with the same plant 
host were previously not studied. Multiple mutualism effects are known to play a role in the net outcome of a system including all partners interacting with the focal mutualist or host (Stanton, 2003; Afkhami et al., 2014), although empirical data demonstrating these complex multispecies interactions are lacking. This study adds the novel finding that plants with rhizobial symbiosis secrete less extrafloral nectar $(\mathrm{EFN})$ and recruit fewer ants.

Conflict among mutualisms within the same plant host are not surprising given maintaining partner cooperation can be costly (Bronstein, 2001; Werner et al., 2014). Any net outcome resulting in reduced fitness for the host plant can be considered a cost, including ecological costs. The most well-known ecological cost in ant-plant mutualisms is the potential to deter beneficial pollinators. A recent study shows bees pollinated fewer plants with ant decoys relative to plants with circular controls, indicating bees can perceive ant presence as a dangerous threat (Assunção et al., 2014). This conflict may depend on how well ants defend their plant host, usually with more aggressive behavior. In some cases only these more aggressive ants have a negative impact on the plant-pollinator mutualism, posing a greater ecological cost with increasing mutualistic quality of ant partners (Ness, 2006). 
Rewarding mutualistic partners is intrinsically costly as the relationship is generally established based on sharing resources. Extrafloral nectaries require large amounts of carbon when EFN production is induced, but the question of how costly EFN production actually is to the plant remains unanswered. In general, inducible defenses are considered to have evolved as a mechanism to reduce the cost of defense by conserving resources until the plant is under attack. The inducible nature of EFN, as well as the cellular machinery involved in inducing EFN via jasmonic acid through the octadecanoid pathway, indicates that there is a cost to the plant in producing nectar for secretion (Mueller et al., 1993). EFN secretion is dependent on photosynthesis as light regulates jasmonate signaling in the induction of EFN (Radhika et al>, 2010b) and photosynthetic area removal by caterpillar (Li et al., 2012) or by quantitative manipulation (Ballhorn et al., 2014b) reduces the plant's capacity to produce EFN. Other constituents of EFN (e.g. antimicrobials, amino acids) are synthesized and lost along with the photosynthate investment once EFN is secreted (Heil, 2011). There are no known mechanisms by which plants can reabsorb unconsumed EFN. This is one cost often overlooked in indirect defense investment: the external release of resources without recovery, versus the ability to catabolize and reallocate direct defense compounds. Our data contribute to this growing body of evidence showing EFN may not be as "cheap" to produce as was previously 
thought (O'Dowd 1979, Rudgers and Gardener 2004): plants with an additional carbon sink (rhizobia) produced less EFN.

The mechanisms leading to altered plant traits with rhizobia are reasonably driven by the symbiotic exchange of carbon-based photosynthates for organic forms of nitrogen. The benefits of nitrogen for plant nutrition are well-understood (Brockwell et al., 1995), and our study reemphasizes that plant tissues contain more protein when plants are associated with rhizobia. Rhizobia also increase plant defensive traits involving large amounts of nitrogen, including cyanogenesis as observed in our study, which is in line with previous work (Thamer et al., 2011). The cost of maintaining nitrogenfixing rhizobia nodules may also significantly alter plant physiology as Peoples et al. (1986) reported about $20-30 \%$ of plant photosynthates are allocated to the microbial partner. Thus, high quantities of photosynthates are required to maintain rhizobial mutualism, and in environments that limit photosynthesis, the plant's obligation to rhizobia can lead to carbon starvation in other plant tissues (Tsikou et. al 2013). This same phenomenon occurs with arbuscular mycorrhizal fungi, another group of microbial symbionts requiring photosynthates from plant hosts, which reduced plant EFN production in a previous study (Laird \& Addicott, 2007). Therefore, a similar resource allocation constraint between two carbon sinks: rhizobia and EFN (Kiers et al., 2003; Mondor et al., 2006) may have driven the decrease in 
EFN in our study.

The observed decrease in EFN on plants with rhizobia is especially interesting given the simultaneous increase in aboveground biomass and protein content—-traits indicating higher photosynthetic ability and carbon availability. However, biomass did not significantly interact with EFN in the structural equation models (Fig. 2), whereas biomass did directly link to both protein and cyanogenesis. An alternative interpretation of the reduced EFN on plants with rhizobia might be an interaction among plant traits. Cyanogenesis as a direct defense is innately more reliable than indirect defenses, which depend on insect behavior (Ballhorn et al., 2008b). Rhizobia may provide enough nitrogen to enable plants to defend themselves via cyanogenesis, whereas nitrogen availability is limited for plants without rhizobia (Thamer et al., 2011), resulting in the observed low cyanogenic potential. In this case, plants may boost their defense system by secreting EFN. Loss of photosynthates to nectaries would not seem to offer additional benefit to a highly cyanogenic plant. The mechanisms regulating EFN production remain widely elusive (Heil, 2011), and insight in this area would augment plant defense theory.

Plant defense versus growth has been a study of interest without universal resolve for many years (Herms \& Mattson, 1992; Stamp, 2003; 
Agrawal \& Fishbein, 2006; Heath et al., 2014), and several hypotheses seek to explain constraints leading to the observed patterns of plant defense (reviewed in Stamp 2003). Aligning these hypotheses into one congruent theory of defense may not be possible without incorporating all biotic influences including mutualistic interactions (Heath et al., 2014). Our data suggest belowground symbiotic rhizobia critically impact lima bean defense strategy, which consequentially affected ant recruitment. The implications of reduced ant attraction to plants with rhizobia have not yet been considered in empirical research. Ants drive many ecological processes including nutrient cycling via effective foraging techniques (Verchot et al., 2003), and mutualistic interactions such as aphid-farming (Schowalter, 1981). As both players—rhizobia and ants—occur almost ubiquitously in terrestrial habitats, the effects of rhizobia on plant traits affecting ant behavior likely have a far-reaching impact. Thus, interactions among plant mutualists may have strong and widely overlooked impacts on plant ecology. 


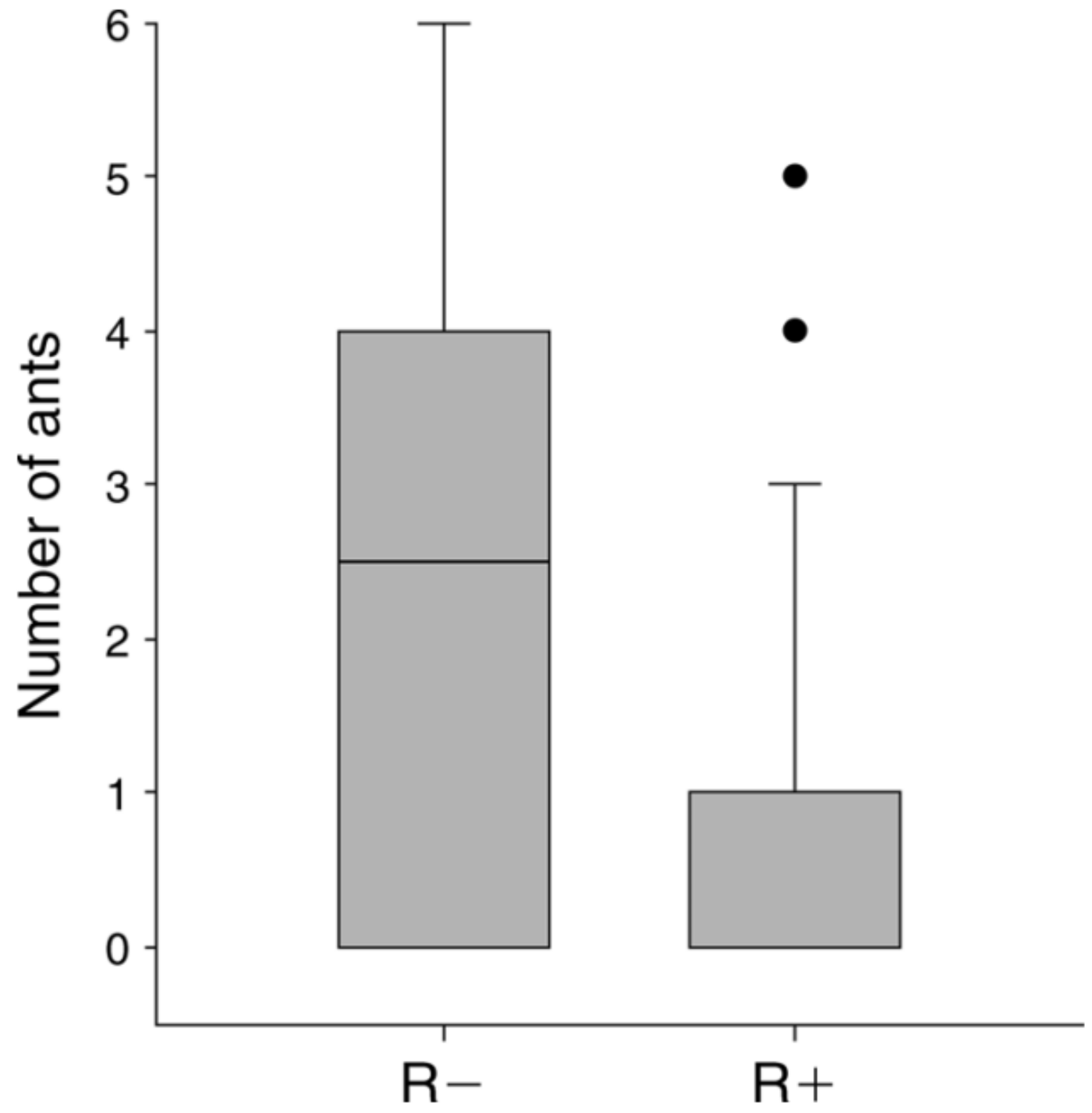

Figure 7.1 Ant recruitment onto Phaseolus lunatus plants with and without rhizobia. Values are given as box plots of ants counted $8 \mathrm{~h}$ after extrafloral nectar (EFN) induction; center line indicates median values, ends of boxes indicate upper and lower quartiles. The error bars represent 90th percentile values (as the lower quartile reached zero, no 10th percentile values were possible), and the dots represent extreme values above the $90^{\text {th }}$ percentile. Treatments $\mathrm{R}$ ? and $\mathrm{Rp}$ represent rhizobia-free plants and rhizobia-inoculated plants, respectively. 


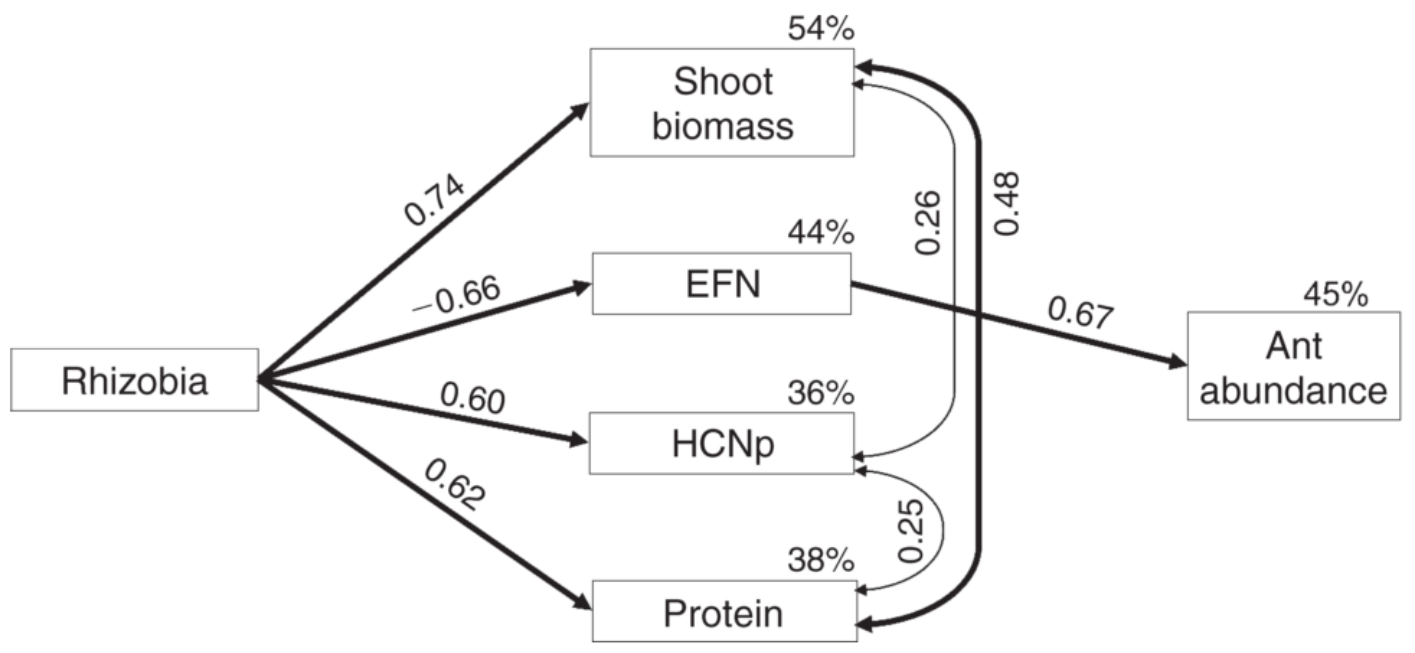

Figure 7.2 Final reduced structural equation model for the relationship between rhizobia, aboveground P. lunatus plant traits (including cyanogenic potential, HCNp, and plant nutritive quality, expressed as total soluble protein), and ant abundance. Thin lines indicate marginally significant $(\mathrm{P}$, $0.1)$ and thick lines indicate significant $(P, 0.05)$ relationships. Numbers on arrows are standardized path coefficients. Percentages above endogenous variables indicate the variance explained by the model (R2). The data did not significantly deviate from the model (see Appendix C for unstandardized estimates and model fit indices). 

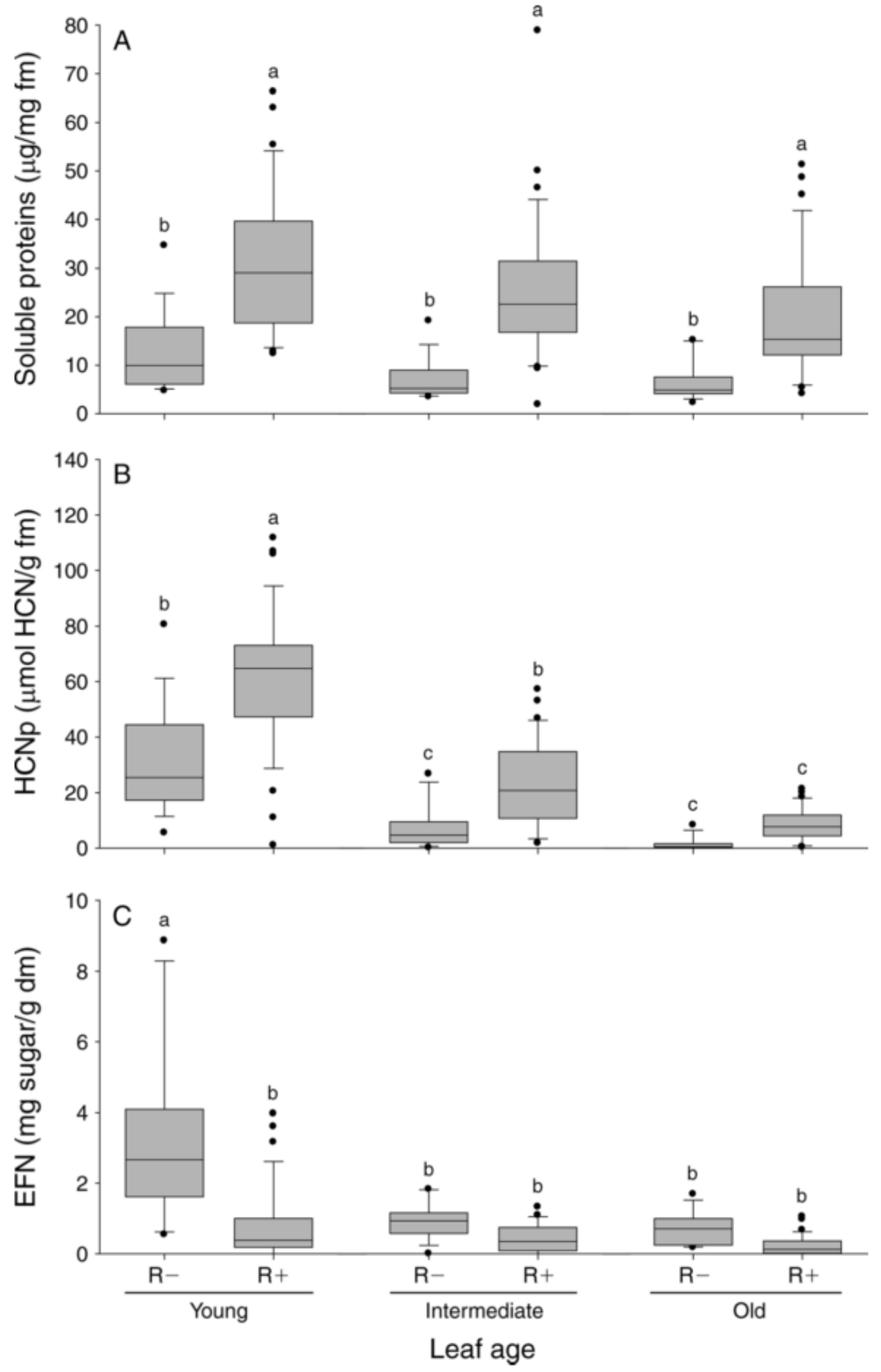
Figure 7.3 P. lunatus plant traits with and without rhizobia for young, intermediate, and mature leaves. Rhizobia presence/absence is indicated by R+/R-. Different lowercase letters indicate significant differences following Tukey's post hoc tests $(\mathrm{P}, 0.05)$ from a split-plot linear mixed model with plant individual as random main plot factor and leaf age as subplot factor within plant individual. Values are presented as boxplots; center line indicates median values, ends of boxes indicate upper and lower quartiles. The error bars represent 10th and 90th percentile values, and the dots represent extreme values below or above the 10th and 90th percentiles. (A) Soluble protein concentration, measured in lg protein/mg leaf fresh mass (fm). (B) HCNp, measured in lmol/L hydrogen cyanide/g leaf fresh mass. (C) EFN secretion, measured in $\mathrm{mg}$ sugar/g leaf dry mass $(\mathrm{dm})$ 


\section{Chapter 8: Ants distinguish between extrafloral nectar scents}

In preparation as: Godschalx AL, A Diethelm, TL Smagula, TN Rosenstiel, and DJ Ballhorn. Ants are differentially attracted to species-specific extrafloral nectar scents. In preparation for publication in New Phytologist.

\section{Summary}

- Many plants respond to attack by secreting sugar-rich nectar-not for pollination—but for protection. Aggressive ants and other predators patrol leaf surfaces in response to extrafloral nectar (EFN) secretion, but how predators respond in a timely fashion to EFN as an inducible defense in plants has not been understood mechanistically.

- To test the potential for EFN volatiles to influence ant behavior, we conducted bioassays and characterized the EFN scent profiles of three species with inducible EFN: lima bean, Phaseolus lunatus; passion vine, Passiflora edulis; and cherry, Prunus avium.

- Here we demonstrate Lasius niger ants respond positively to volatile constituents of EFN, and are most attracted to recent host plant EFN. Volatile profiles from three facultative ant-plants show a wide diversity of putative semiochemicals.

- Volatile-based communication fills a gap in our understanding of how EFN rewards mediate ant-plant interactions in response to induced signals. EFN scents contribute to potential mechanisms underpinning 
efficiency of indirect defense communication, and on a broader time scale, maintaining non-obligate mutualistic interactions.

Keywords: Ant-plant, coevolution, extrafloral nectar (EFN), indirect defense, mutualism, nectar chemistry, scented nectar, volatile organic compounds (VOCs)

\section{Introduction}

Cooperation in nature has received well-deserved fascination in part because the forces that maintain a mutually beneficial outcome are constantly confronted with less cooperative partners (Jones et al., 2015). By investigating the chemical signals mediating relationships among organisms, we gain insight into the communication interface between parties that may influence mutualistic behavior. The ant-plant mutualism is a well-studied relationship in nature; plants provide sugar-rich extrafloral nectar (EFN), and in doing so, recruit ants to patrol herbivore-prone surfaces. Protection by ants is an effective defense, and secreting EFN affords plants protection against consumption without investing extensively in costly direct defense metabolism (McLain, 1983). However nectar is not a free resource (Southwick, 1984), and plants with reduced photosynthetic area or with a strong carbon sink secrete less EFN (Li et al>, 2012; Ballhorn>et al>, 2014b; Godschalx >et al>, 2015a). While floral nectaries can resorb nearly all 
unconsumed nectar, EFN resorption has not been shown (Nepi et al., 2001; Heil, 2011). Likely as a mechanism to conserve resources, most EFNsecreting plants induce EFN secretion only in response to chemical signals that indicate the plant is under attack, forming a facultative, non-obligate relationship with ants in the surrounding area (Heil et al., 2001). By contrast, obligate ant-plants maintain a reliable daily EFN production routine for resident bodyguards, and can increase mutualistic benefit from their ant defenders by increasing resource investment in EFN (González-Teuber et al., 2012). Increasing mutualistic help from ants as a facultative ant-plant might require a mechanism for effective signaling. It is intriguing to speculate that plants use a recruitment signal from EFN itself that contributes to directly alerting and attracting defensive ants, but this has yet to be explicitly examined (González-Teuber \& Heil, 2009).

Nectar can be scented, as is the case for various floral nectars, which manipulate pollinator behavior to increase floral visit frequency and pollinator specificity (Raguso, 2004; Kessler \& Baldwin, 2007). Recently it has been shown that EFN from flower stipules can also be scented, including volatiles unique to the nectar resource not found from surrounding tissues (Hoffmeister \& Junker, 2017). If EFN associated with vegetative structures also contain volatile cues, although not yet studied, then ants may be able to use these as advertisement. Floral nectar-robbing ants respond to several 
volatile cues, many of which are different from pollinator attractants (Kessler $\&$ Baldwin, 2007). Ant-attractive volatile nectar signals may be possible within beneficial ant-plant relationships because plants use leaf volatile signals to manipulate ant partner behavior and more efficiently direct ant patrol attention towards particular tissues that need more protection (Brouat et al., 2000). Ants respond with increased foraging and associating nectar food rewards to volatile signals from plant tissues (Wanjiku et al., 2014). How ants specifically find EFN has not been determined; ants may find EFN sources simply by chance or, alternatively, by using visual or olfactory and contact chemosensory compounds to locate EFN.

To test the potential for ants to respond to olfactory cues directly from EFN, we designed a custom food choice arena, which elevated black gardener ants (Lasius niger) on a screen above three droplets, identical in appearance, but out of reach for direct contact. We measured ant responses as ants could choose freely among the volatile headspace zones. We conducted two series of choice experiments offering a set of i) water, aqueous sugar solution (sucrose), and EFN droplets from a single plant species (either Phaseolus lunatus, Passiflora edulis, or Prunus avium; all facultative ant-plants), or ii) EFN from all three plant species simultaneously. We conducted an additional series of bioassays containing all three EFNs, but varying plant host from which we collected conspecific ants (all L. niger) to determine whether ants 
can recognize specific EFN cues associated with particular hosts. To test for volatile constituents in leaf stipule EFN and identify differences in volatile chemistry among each species' EFNs, we exposed solid phase microextraction (SPME) fibers to EFN headspace, thermally desorbed putative EFN VOCs with gas chromatography time-of-flight mass spectroscopy (GC-TOFMS), and utilized total ion current peak areas (TIC) for multivariate analysis via BrayCurtis distance-based multidimensional scaling (MDS) to determine whether suites of compounds were specific to each nectar-secreting species. Because all host plants in this study are facultative ant-plants, we hypothesized each species' EFN would contain a volatile signal. If vegetative structureassociated EFN is scented and ants show attraction to EFN over sucrose or water droplets, plants may be able to more directly manipulate the signals communicated to ants providing the defensive service, therefore, more efficiently secrete EFN as a defensive trait.

\section{Materials and Methods}

\section{Extrafloral Nectar (EFN) Collection}

EFN from Prunus avium, Passiflora edulis, and Phaseolus lunatus was collected between March - August 2015 from outdoor plants on a private property in Raleigh Hills (DJ Ballhorn, Portland, OR). Plant species were selected as representatives from different plant families that have contributed to our understanding of EFN regulation: Prunus avium (Pulice \& 
Packer, 2008), Phaseolus lunatus (Radhika et al., 2010b), and Passiflora edulis (Izaguirre et al., 2013), all of which are reviewed in (Heil, 2015). Because each species produces EFN in response to damage-associated cues, 24 hours prior to collection, plants sprayed with $1 \mathrm{mmol} / \mathrm{L}$ jasmonic acid, an established signal for facultative-ant plants that only secrete EFN when induced (Kost \& Heil, 2008). Ants and other nectar-consumers were excluded using a mesh bag and a ring of TangleFoot around the base of the plant or branch. EFN was collected via surface tension without contacting surrounding plant tissue using glass microcapillary tubes (PCR Micropipettes

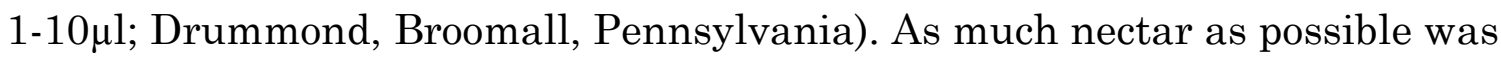
collected for chemical analysis, volumes for each sample are listed in Table $\mathrm{S} 1 ; 2 \mu \mathrm{L}$ per sample were collected for bioassay experiments.

\section{Ant Bioassays}

Lasius niger ants were collected from four different sources at the same site, including naïve ants from not associated with any of the three focal species, and ants found foraging on Prunus avium, Passiflora edulis, and Phaseolus lunatus plants on August, 18 $8^{\text {th }}, 2015$. Ants were placed on a screen (mesh size $0.381 \mathrm{~mm}, 7.5 \mathrm{~cm}$ diameter) custom-fitted to a plastic ring that fit inside of a $9 \mathrm{~cm}$ petri dish, and supported ants raised $0.5 \mathrm{~cm}$ above the petri dish bottom. Screens were rinsed between ants for one minute in ethanol followed by one minute in acetone. Below the screen mesh and out of reach for the ants, three 
droplets $(2 \mu \mathrm{L})$ were evenly spaced, and each one was encircled from below by $18 \mathrm{~mm}$ diameter ring to indicate the "headspace zone" for each nectar droplet (Fig. 1). Bioassays were conducted for four minutes, recording length of time ants spent within each droplet's headspace zone. Ants could not directly contact the droplets. Naïve ant bioassays included either i) a water droplet, aqueous sucrose, and EFN from one of the facultative ant-plants, Phaseolus lunatus, Prunus avium, and Passiflora edulis, or ii) all three EFNs simultaneously ( $n=10$ Lasius niger ants per treatment). Prior-experience bioassays exposed ants to EFN from all three plant species, using ants collected from each plant species ( $n=10$ Lasius niger ants per plant species).

\section{Volatile organic compound (VOC) collection}

For both vine species (P. edulis and P. lunatus), total EFN volume from each plant was collected, and for the woody tree species (P. avium), EFN within the reachable range was quantitatively collected with microcapillaries as described above. Volumes for each sample are recorded in Table S1. On the same day, within one hour following collection, EFN was expelled into $2 \mathrm{~mL}$ glass vials with $9 \mathrm{~mm}$ silicone/PTFE septa screw caps (VWR) and exposed to Solid Phase Microextraction (SPME) fibers (Polydimethylsiloxane/Divinylbenzene (PDMS/DVB), $\mathrm{d}_{\mathrm{f}} 65 \mu \mathrm{m}$, needle 24ga, StableFlex, Sigma Aldrich). EFN headspace was collected for 1 hour while vials were submerged in a $30^{\circ} \mathrm{C}$ water bath to volatilize all potential volatile 
constituents that may be emitted at canopy temperatures in nature. Samples were iteratively collected over the course of several months from available plants, resulting in sample sizes of $\mathrm{n}=19,14$, and 6 pooled $\mathrm{EFN}$ samples for $P$. lunatus, $P$. avium, and $P$. edulis respectively. Controls were created by opening $2 \mathrm{~mL}$ vials for the same duration and held in microsite locations near each extrafloral nectary to control for ambient and surrounding foliar VOCs. SPME fibers were inserted into a Pegasus GC-TOFMS system (LECO) for thermal desorption and gas chromatography- mass spectroscopy for time-offlight (TOF) analysis.

\section{Volatile Data Processing Analysis}

All peaks from all samples including blanks were sorted by retention time, and unique masses for each compound were compared against all other samples to identify putative volatiles that consistently appear at a similar retention time. Each peak's mass spectrum was manually matched to the most appropriate library hit to confirm identity as best as possible without known standards for all 87 compounds in our final analysis. Compounds prevalent in blank samples and SPME fiber control runs were removed from the final analysis.

\section{Statistical Analyses}

Volatile TIC peak area data from GC-TOFMS were analyzed using Metric Multidimensional Scaling (MDS) with Bray-Curtis distance (Paliy \& 
Shankar, 2016) and ANOSIM analysis of dissimilarity in R Studio (R Core Team, 2015). Ant choice behavior was assessed by conducting one-way ANOVAs for the single-nectar assay as well as for the three nectar assays, with a separate analysis for ants responses from each of the four collection sites (three plant species and one naïve treatment). Ant response times were natural log-transformed to meet ANOVA assumptions of normality and passed tests for equal variance. Differences among duration in dropletspecific ant attraction were distinguished using a Holm-Sidak Multiple Comparisons test. ANOVAs and post-hoc analyses were conducted using SigmaPlot 12.0.

\section{Results}

\section{Ant responses to EFN headspace}

Ants made a clear choice in all bioassays, spending significantly more time within the zone above the extrafloral nectar $(\mathrm{EFN})$ sample compared with the water or sucrose controls (Fig. 2a-c). In addition to consistently identifying EFN over controls, all three EFNs elicited unique responses from ants collected from a non-focal plant source. Naïve L. niger ants without prior experience spent significantly different amounts of time in headspace zones above each plant species' EFN (Fig. 2d). P. edulis EFN was most attractive to naïve ants in bioassays, followed by EFN from $P$. lunatus, with EFN from $P$. avium drawing the least amount of ant attention. This difference in 
attractiveness among different plants' EFN led us to test whether an ant with prior experience on any of the tested plant species could influence olfactory-based decisions. When L. niger ants collected from each of the three plant species were assayed, the most attractive nectar in all cases was EFN from the source plant species (Fig. 3).

\section{EFN Volatile Collection}

The number of compounds included in the final analysis varied by plant species, with $P$. edulis EFN containing 29 compounds, less than half the number of compounds from $P$. lunatus EFN, with 71 compounds, or P. avium EFN, with 62 (compounds listed in Table 1). The number of compounds within each functional class varied by plant species as well. The largest number of compounds within a particular class were the 13 different monoterpenes found in P. avium. P. avium was also the only species to produce octadecanoid products, methyl jasmonate and cis-jasmone. Other compounds unique to $P$. avium included benzyl alcohol, benzyl benzoate, camphor, and a few other monoterpenes, alcohols and ketones, contributing to the 13 compounds found only in $P$. avium. The greatest number of unique compounds, 20, were found in P. lunatus. as well as the highest number of total and unique $\mathrm{N}$-containing volatiles, including indole, isothiocyanate, nonanamide, trans-pinocarveol and other monoterpenes, 3-hexen-1-ol and other alcohols, an alkene, ester, and several organic acids-more organic 
acids than any other species. $P$. edulis EFN has the fewest organic acids. The three compounds unique to $P$. edulis include an alkene, a cyclic compound and 4-methyl- 2-heptanone. Compounds in common among all three species' EFN volatile profiles include ketones, sulcatone (6-methyl-5-hepten-2-one); monoterpenes, 2-Octene, 2,6-dimethyl- and (1R,2R,3S,5R)-(-)-2,3-Pinanediol; sesquiterpenes, 5,9-Undecadien-2-one, 6,10-dimethyl-, (E)- and farnesol isomer a, along with a few aromatics, alcohols and ketones. Methyl salicylate was present in EFN of both $P$. avium and P. lunatus, but not $P$. edulis, along

with several linalools. Peak areas for all 87 compounds in a multidimensional scaling analysis (MDS, stress value=0.236; Fig. 4) were significantly explained by plant species from analysis of similarity (ANOSIM, $R=0.6003$, $p<0.001)$.

\section{Discussion}

Plants recruit ants as protectors in nearly all terrestrial ecosystems. Here we describe an additional facet of plant-ant communication by presenting evidence of scented extrafloral nectar mediating ant recognition and attraction. Ants were most attracted to headspace zones above EFN compared to non-EFN droplets, demonstrated preferences between all three plant species' EFNs, and recognized host plant EFN from volatile headspace of EFN. Strong responses from ants is not surprising given the body of work surrounding ant chemical communication (Inui \& Itioka, 2007; Youngsteadt 
et al., 2008; Holman et al., 2010; Wanjiku et al., 2014). Hymenopteran associative behavior is not surprising as learning behavior occurs throughout the order (Turlings et al>, 1993b; Arenas \& Farina, 2012; Luo>et al>, 2013). In learning nectar cues, our data corroborate with known nectar-seeking behavior in predators. Cursorial spiders respond to chemicals cues-even synthetic experimental cues such as vanilla-more rapidly than unscented sucrose, but only after having consumed that nectar, indicating spiders can learn nectar chemical cues from foraging (Patt \& Pfannenstiel, 2008). Associating nectar food rewards increases ant responses to plant volatiles from various plant organs in Cashew tree (Wanjiku et al., 2014). Experience with EFNs on cotton plants led parasitoid wasps to select EFN-bearing plants over plants without nectaries in whole-plant flight tunnel experiments, and naïve wasps exhibited close-range olfactory-based behavior in selecting EFN over honey and sucrose (Röse et al., 2006). Spider, ant, and wasp responses to EFNs support our observation of olfactory-based selection behavior in ant nectar-foraging, and suggest potential for ants to learn cues associated with EFN.

Without direct contact, and after maintaining consistent visual cues for all samples in our bioassays, ant preferences for the zones above EFN droplets strongly suggest that foliar EFNs contain volatile information. This hypothesis is supported by volatile profiles of EFNs associated with floral 
tissue in Vicia faba containing volatiles that largely, yet do not completely reflect VOCs of surrounding plant tissues (Hoffmeister \& Junker, 2017). Linalool is present in both floral nectar and EFN scent bouquets of Vicia faba, which increased in response to JA-induction, resulting in pollinators losing significant preferences between floral nectar and flower-associated EFN that were clearly observed in uninduced plants (Hoffmeister \& Junker, 2017). Similarly, we treated all plants with JA prior to analyzing VOCs, and we also found linalool along with several derivates in two out of three plant species' EFN (Table 1). P. avium is one of these two species, and when compares against the other two species' EFN, P. avium attracted the least amount of ant attention. P. avium EFN, contained the most known floral volatile compounds, which may explain why ants without prior experience were least attracted to $P$. avium EFN among all three species, as many floral volatiles typically deter ants (Kessler \& Baldwin, 2007; Willmer et al., 2009). Interesingly, $P$. avium was the only plant species' EFN to contain any octadecanoid products, which included methyl jasmonate and cis-jasmone. Cis-jasmone also plays an important role as a semiochemical in plant indirect defense signaling, deterring aphids while attracting ladybeetle and aphid parasitoids (Birkett et al., 2000). By contrast, the most attractive EFN among all three species was P. edulis, despite emitting the fewest overall and only three unique compounds. Fewer compounds may be important to 
maintain a high signal to noise ratios if specific compounds are the most important for ant attraction, or if smaller peaks play key roles in bioactivity (D'Alessandro et al., 2009). Even if a semiochemical is present in all three species, the number of compounds interacting in the volatile bouquet may influence biological responses. One example of an interesting compound present in all three, but potentially less diluted in $P$. edulis could be sulcatone (6-methyl-5-hepten-2-one). Sulcatone serves as a semiochemical in wheat to recruit aphid parasitoids (Liu et al., 2009). Alternatively, among the three compounds unique to $P$. edulis, one is a ketone 4-methyl 2-heptanone, a known ant alarm (Moser et al., 1968). Ant secretions containing 4-methyl-2heptanone putatively have antimicrobial activity (de Lima Mendonça et al., 2009). The striking absence of organic acids in P. edulis may imply vastly different microbial communities in each species EFN, compared against $P$. lunatus EFN with seven different organic acids, four of which are only in $P$. lunatus. Volatiles unique to $P$. lunatus include several N-containing volatiles, which is interesting because lima bean is the only legume used in this study, and plants did have symbiotic, nitrogen-fixing rhizobia. Previous rhizobiamediated plant chemistry data shows indole, along with methyl salicylate, are the only two volatiles observed that rhizobia affect positively (Ballhorn et al>, 2013c). Methyl salicylate was present in EFN volatiles from P. lunatus, as well as in $P$. avium. which, as mentioned above, was the sole methyl 
jasmonate-emitter. Given well-established hormonal crosstalk between these two pathways, JA and SA,(Wei et al., 2014b), along with the myriads of potentially relevant compounds for ant-signaling, future chemical ecology avenues to understand EFN scents is only beginning.

The ecological significance and evolutionary implications of plants using EFN scent to communicate with ant defenders may parallel our understanding of plants using floral nectar scent to communicate with pollinators. Not all floral nectars are scented (Raguso, 2004), so it would be expected that not all EFNs are scented either, likely explaining parasitoid wasp indifference to EFN without being fortified with floral nectar in a Y-olfactometer experiment, which elicited a wasp response (Géneau et al., 2013). Obligate ant-plant with diurnal rhythms of secretion may not be expected to investing in scented EFN, however, these plants have EFN with extensive exoenzyme production and antimicrobial activity (González-Teuber et al., 2009), so defensive volatiles may dominate Acacia EFN scents if present. When floral nectar is scented, the ecological functions range from defense against exploitation or microbial contamination, to attraction as an honest signal of nectar rewards (Raguso, 2004). Ant aggressiveness can be manipulated by plants through nectar composition (Ness et al., 2009), and levels of secondary metabolites in nectar below toxic thresholds can increase animal drugseeking behavior (Grasso et al., 2015) and memory (Wright et al., 2013). 
Given the potential for floral volatiles to influence ant behavior (Willmer et al., 2009), volatiles in EFN may be a mechanism for plants to manipulate the aggressiveness of their ant defenders through biological activity of specific scents, and while this has not been studied, future results could uncover another level of detail in this specific partner manipulation to maintain mutualisms.

Scented nectar in both systems, floral and extrafloral, could be truly adaptive plant traits, intentionally-loaded volatiles into nectar, otherwise volatiles could also be co-opted from pollinator or defender responses microbial metabolism or volatiles emitted by neighboring plant cells. Aqueous sugar solutions absorbed floral tissue volatiles from nectarless Magnolia grandiflora, but nectar from Oenothera primiveris and Agave palmeri produced VOC profiles that differed from surrounding tissues, consistent with EFN volatiles in V. faba (Raguso, 2004; Hoffmeister \& Junker, 2017). Microbial metabolism may contribute to differences in tissue versus nectar VOCs, but byproducts depend on the sugar and amino acid constituents of the nectar as well as the identity and composition of the microbial community. Nectar containing bacteria, as opposed to yeast, reduces bee attraction (Good et al., 2014) and reduced pollination success in hummingbird-pollinated Mimulus aurantiacus (Vannette et al., 2013) because of the bacteria-mediated changes in nectar chemistry. Such shifts in 
microbial community and subsequently, nectar chemistry, can be a product of ant visitation (de Vega \& Herrera, 2013). If ants transport microorganisms as nectar chemical engineers (Vannette \& Fukami, 2016), or contribute glandular secretions with antimicrobial activity (de Lima Mendonça et al., 2009) the potential for ants to contribute to EFN scent creates a two-way mode of chemical communication in ant-plant interactions. Interesting future research could investigate plant- and microbial- derived VOCs in EFN, as well as the role of insect vectors in shaping scent phenotype to understand the degree to which each organism "controls" this trait and influences the other players in this ant-plant mutualism.

With chemical ecology reinforcing aqueous sugar rewards for predators, the evolution of scented nectar is convergent. Both floral and extrafloral nectars can use scents to attract mobile insect mutualists for pollination or defensive services. Interestingly, nectar in both cases can be strongly regulated by jasmonic acid signaling pathways, which has been more extensively studied for extrafloral nectar given its role in indirect defense and the association of JA with defensive plant chemistry. Floral nectar production is linked to endogenous JA levels and can be manipulated by exogenous JA or JA pathway inhibition (Radhika et al., 2010a). By inducing scented EFN in response to cues indicating herbivory, and if ants seek out familiar host EFN odors as, is the case with pollinators and floral nectar scents (Raguso, 2004; 
Howell \& Alarcón, 2007), facultative ant-plants may use scents as honest signals to optimize investment while recruiting and maintaining ant bodyguards, which would be consistent with leading plant defense hypotheses (Wäckers \& Bonifay, 2004; Bixenmann et al., 2013) However, scented nectar in flowers or EFNs are not without ecological costs, as ants can act as nectarrobbers, and pollinators can be equally attracted to floral and EFN scents, which may have important impacts on plant fitness, and open a myriad of avenues for understanding plant-herbiore-predator-pollinator interactions (Junker \& Blüthgen, 2008; Kessler \& Halitschke, 2009b; Hoffmeister \& Junker, 2017). Here we show species-specific EFN fragrances and ant attraction to each suite of volatiles. EFN volatiles specific to each plant species were recognizable and attractive to ants.

Scented nectar, playing a role in pollination biology—and now also in plant defense biology—is an example of convergent evolution of a plant trait manipulating insect behavior for these two services using the intriguing combination of a nectar food reward with volatile chemical cues. 
Table 8.1 Species differences in EFN volatiles vary by chemical class. All compounds included in final analysis are listed, separated by species in separate columns, and by chemical class by rows. Values below the dotted line for each class show the number of volatiles from that class each species' EFN contains. Values at the bottom of the table show total compounds present for each species, and number of compounds unique to only that species. Unique compounds are indicated throughout the table in bold. Each compound is only listed once, even if the compound could fit into multiple classes. 


\begin{tabular}{|c|c|c|c|}
\hline & Prunus avium & Phaseolus lunatus & Passiflora edulis \\
\hline \multirow[t]{2}{*}{ Monoterpenes } & $\begin{array}{l}\text { 1S-à-Pinene } \\
\text { Limonene } \\
\text { Myrcenol } \\
\text { cis-Linalool Oxide } \\
\text { 2-Octene, 2,6-dimethyl- } \\
\text { Linalool } \\
\text { Hotrienol } \\
\text { 3-Methyl-3-cyclohexen-1-one } \\
\text { á-Pinone } \\
\text { Camphor } \\
\text { Epoxylinalol } \\
\text { 3,7-Octadiene-2,6-diol, 2,6- } \\
\text { dimethyl- } \\
\text { - } \\
\text { (1R,2R,3S,5R)-(-)-2,3- } \\
\text { Pinanediol } \\
\text { - } \\
\text { - }\end{array}$ & $\begin{array}{l}\text { 1S-à-Pinene } \\
\text { Limonene } \\
\text { Myrcenol } \\
\text { cis-Linalool Oxide } \\
\text { 2-Octene, 2,6-dimethyl- } \\
\text { Linalool } \\
- \\
\text { 3-Methyl-3-cyclohexen-1-one } \\
- \\
- \\
\text { Epoxylinalol } \\
- \\
\text { trans-Pinocarveol } \\
\text { (1R,2R,3S,5R)-(-)-2,3-Pinanediol } \\
\text { (1R)-(-)-Camphorquinone } \\
\text { Bicyclo[2.2.1]heptan-3-one, 2- } \\
\text { benzyloxy-1,7,7-trimethyl- }\end{array}$ & $\begin{array}{l}- \\
- \\
- \\
- \\
\text { 2-Octene, 2,6- } \\
\text { dimethyl- } \\
- \\
- \\
- \\
- \\
- \\
- \\
- \\
- \\
\text { (1R,2R,3S,5R)-(-)-2,3- } \\
\text { Pinanediol } \\
- \\
-\end{array}$ \\
\hline & 13 & 12 & 2 \\
\hline \multirow[t]{2}{*}{ Sesquiterpenes } & $\begin{array}{l}\text { ç-Elemene } \\
\text { 5,9-Undecadien-2-one, 6,10- } \\
\text { dimethyl-, (E)- } \\
\text { - } \\
\text { Farnesol isomer a }\end{array}$ & $\begin{array}{l}\text { ç-Elemene } \\
\text { 5,9-Undecadien-2-one, 6,10- } \\
\text { dimethyl-, (E)- } \\
\text { Farnesol } \\
\text { Farnesol isomer a }\end{array}$ & $\begin{array}{l}- \\
\text { 5,9-Undecadien-2-one, } \\
\text { 6,10-dimethyl-, (E)- } \\
- \\
\text { Farnesol isomer a }\end{array}$ \\
\hline & 3 & 4 & 2 \\
\hline \multirow[t]{2}{*}{ Octadecanoid } & $\begin{array}{l}\text { Methyl jasmonate } \\
\text { cis-Jasmone }\end{array}$ & $\begin{array}{l}- \\
-\end{array}$ & $\begin{array}{l}- \\
-\end{array}$ \\
\hline & 2 & 0 & 0 \\
\hline \multirow[t]{2}{*}{ Benzenoids } & $\begin{array}{l}\text { Methyl salicylate } \\
\text { 3,4-Dimethylbenzamide } \\
\text { Benzaldehyde } \\
\text { Benzyl Alcohol } \\
\text { Benzyl Benzoate } \\
\text { Acetophenone } \\
\text { Indan, 1-methyl- } \\
\text { Benzenemethanol, à,à- } \\
\text { dimethyl- } \\
\text { Benzophenone } \\
\text { Benzene, (1-butylheptyl)- } \\
\text { - } \\
\text { - } \\
\text { 1H- } \\
\text { Cyclopentano[c]coumarine, } \\
\text { 2,3-dihydro-7-benzyloxy- }\end{array}$ & $\begin{array}{l}\text { Methyl salicylate } \\
\text { 3,4-Dimethylbenzamide } \\
\text { Benzaldehyde } \\
- \\
\text { - } \\
\text { Acetophenone } \\
\text { Indan, 1-methyl- } \\
\text { Benzenemethanol, à,à-dimethyl- } \\
\text { Benzophenone } \\
\text { Benzene, (1-butylheptyl)- } \\
\text { Benzene, (1-ethylnonyl)- } \\
\text { Benzene, (1-pentyloctyl)- } \\
\text { - }\end{array}$ & $\begin{array}{l}- \\
- \\
\text { Benzaldehyde } \\
- \\
- \\
\text { Acetophenone } \\
\text { Indan, 1-methyl- } \\
- \\
- \\
- \\
- \\
- \\
-\end{array}$ \\
\hline & 11 & 10 & 3 \\
\hline
\end{tabular}




\begin{tabular}{|c|c|c|c|}
\hline \multirow[t]{2}{*}{ Alcohols } & $\begin{array}{l}\text { Amylene hydrate } \\
- \\
- \\
- \\
\text { 2-Butene-1,4-diol, (Z)- } \\
\text { 3-Hexanol, 5-methyl- } \\
\text { 1-Propanol, 2,2'-oxybis- } \\
\text { 3-Methyl-hepta-1,6-dien-3-ol }\end{array}$ & $\begin{array}{l}\text { Amylene hydrate } \\
\text { 1-Butanol, 3-methyl- } \\
\text { 3-Hexen-1-ol } \\
\text { (R)-(-)-2-Pentanol } \\
\text { 2-Butene-1,4-diol, (Z)- } \\
\text { 3-Hexanol, 5-methyl- } \\
\text { 1-Propanol, 2,2'-oxybis- } \\
\text { - }\end{array}$ & $\begin{array}{l}\text { Amylene hydrate } \\
- \\
- \\
- \\
- \\
\text { 3-Hexanol, 5-methyl- } \\
- \\
-\end{array}$ \\
\hline & 5 & 7 & 2 \\
\hline \multirow[t]{2}{*}{ Aldehydes } & $\begin{array}{l}\text { Methacrolein } \\
\text { Hexanal } \\
\text { Heptanal } \\
\text { Nonanal } \\
\text { Decanal }\end{array}$ & $\begin{array}{l}\text { Methacrolein } \\
\text { Hexanal } \\
\text { Heptanal } \\
\text { Nonanal } \\
\text { Decanal }\end{array}$ & $\begin{array}{l}- \\
\text { Hexanal } \\
\text { Heptanal } \\
\text { Nonanal } \\
\text { Decanal }\end{array}$ \\
\hline & 5 & 5 & 4 \\
\hline \multirow[t]{2}{*}{ Alkanes } & $\begin{array}{l}\text { Dodecane } \\
\text { Hexadecane } \\
\text { Octadecane, 2-methyl- } \\
\text { Eicosane }\end{array}$ & $\begin{array}{l}\text { Dodecane } \\
\text { Hexadecane } \\
\text { Octadecane, 2-methyl- } \\
\text { Eicosane }\end{array}$ & $\begin{array}{l}\text { Dodecane } \\
\text { Hexadecane } \\
\text { Octadecane, 2-methyl- } \\
\text { Eicosane }\end{array}$ \\
\hline & 4 & 4 & 4 \\
\hline \multirow[t]{2}{*}{ Alkenes } & - & trans-1,4-Hexadiene & $\begin{array}{l}\text { 1-Pentene, 2-methyl } \\
-\end{array}$ \\
\hline & 0 & 1 & 1 \\
\hline \multirow[t]{2}{*}{ Cyclic } & $\begin{array}{l}\text { 3-Cyclopentene-1,2-diol, cis- } \\
\text { 1,3,5,7-Cyclooctatetraene } \\
\text { Furan, tetrahydro-2,2,5,5- } \\
\text { tetramethyl- } \\
\text { - }\end{array}$ & $\begin{array}{l}\text { 3-Cyclopentene-1,2-diol, cis- } \\
\text { 1,3,5,7-Cyclooctatetraene } \\
\text { Furan, tetrahydro-2,2,5,5- } \\
\text { tetramethyl- } \\
\text { - }\end{array}$ & $\begin{array}{l}\text { 3-Cyclopentene-1,2- } \\
\text { diol, cis- } \\
\text { - } \\
\text { Furan, tetrahydro- } \\
\text { 2,2,5,5-tetramethyl- } \\
\text { Furan, 3-methyl }\end{array}$ \\
\hline & 3 & 3 & 3 \\
\hline \multirow[t]{2}{*}{ Esters } & $\begin{array}{l}\text { Hexanedioic acid, bis(1- } \\
\text { methylethyl) ester } \\
\text { Benzoic acid, 2-phenylethyl } \\
\text { ester } \\
\text { - } \\
\text { Dodecyl acrylate } \\
\text { Propanoic acid, 2-methyl-, } \\
\text { butyl ester } \\
\text { Hexanedioic acid, mono(2- } \\
\text { ethylhexyl)ester }\end{array}$ & $\begin{array}{l}\text { - } \\
- \\
\text { n-Dodecyl acetate } \\
\text { Dodecyl acrylate } \\
\text { Propanoic acid, 2-methyl-, butyl ester } \\
\text { Hexanedioic acid, mono(2- } \\
\text { ethylhexyl)ester }\end{array}$ & $\begin{array}{l}- \\
- \\
- \\
- \\
- \\
\text { Hexanedioic acid, } \\
\text { mono(2- } \\
\text { ethylhexyl)ester }\end{array}$ \\
\hline & 5 & 4 & 1 \\
\hline \multirow[t]{2}{*}{ Ketones } & $\begin{array}{l}\text { Methyl vinyl ketone } \\
\text { 3-Hexen-2-one, 5-methyl- } \\
\text { Methyl Isobutyl Ketone } \\
\text { Methylglyoxal } \\
\text { 2,5-Hexanedione } \\
\text { - } \\
\text { Ethanone, 1-(1-cyclohexen-1- } \\
\text { yl)- } \\
\text { Sulcatone } \\
\text { Mesityl oxide } \\
\text { - } \\
\text { - }\end{array}$ & $\begin{array}{l}\text { Methyl vinyl ketone } \\
\text { 3-Hexen-2-one, 5-methyl- } \\
\text { Methyl Isobutyl Ketone } \\
\text { Methylglyoxal } \\
\text { 2,5-Hexanedione } \\
- \\
\text { Ethanone, 1-(1-cyclohexen-1-yl)- } \\
\text { Sulcatone } \\
\text { - } \\
\text { 2-Hexanone, 3-methyl- } \\
\text { 2-Decanone }\end{array}$ & $\begin{array}{l}\text { - } \\
\text { - } \\
\text { Methyl Isobutyl Ketone } \\
\text { Methylglyoxal } \\
\text { 2,5-Hexanedione } \\
\text { 2-Heptanone, 4- } \\
\text { methyl- } \\
\text { - } \\
\text { Sulcatone } \\
\text { - } \\
\text { - } \\
\text { - }\end{array}$ \\
\hline & 8 & 9 & 5 \\
\hline
\end{tabular}




\begin{tabular}{|c|c|c|c|}
\hline \multirow[t]{2}{*}{ Organic Acids } & $\begin{array}{l}- \\
\text { Hexanoic acid } \\
- \\
- \\
\text { Octanoic Acid } \\
- \\
-\end{array}$ & $\begin{array}{l}\text { Butanoic acid, 3-methyl- } \\
\text { Hexanoic acid } \\
\text { 2-Methylheptanoic acid } \\
\text { Benzoic Acid } \\
\text { Octanoic Acid } \\
\text { Nonanoic acid } \\
\text { 2-Propenoic acid, 3-(4- } \\
\text { methoxyphenyl)-, 2-ethylhexyl }\end{array}$ & $\begin{array}{l}- \\
- \\
- \\
- \\
- \\
- \\
\text { 2-Propenoic acid, 3-(4- } \\
\text { methoxyphenyl)-, 2- } \\
\text { ethylhexyl }\end{array}$ \\
\hline & 2 & 7 & 1 \\
\hline \multirow[t]{2}{*}{ S-containing } & Dimethyl sulfone & Dimethyl sulfone & - \\
\hline & 1 & 1 & 0 \\
\hline \multirow[t]{2}{*}{$\mathrm{N}$-containing } & $\begin{array}{l}- \\
- \\
\text { 2-Propenamide, N,N'- } \\
\text { methylenebis- } \\
\text { - }\end{array}$ & $\begin{array}{l}\text { Indole } \\
\text { Isobutyronitrile } \\
\text { 2-Propenamide, N,N'-methylenebis- } \\
\text { Nonanamide }\end{array}$ & $\begin{array}{l}- \\
- \\
\text { 2-Propenamide, N,N'- } \\
\text { methylenebis- } \\
\text { - }\end{array}$ \\
\hline & 1 & 4 & 1 \\
\hline $\begin{array}{l}\text { Total \# of } \\
\text { compounds }\end{array}$ & 62 & 71 & 29 \\
\hline $\begin{array}{l}\text { Unique } \\
\text { compounds }\end{array}$ & 13 & 20 & 3 \\
\hline
\end{tabular}




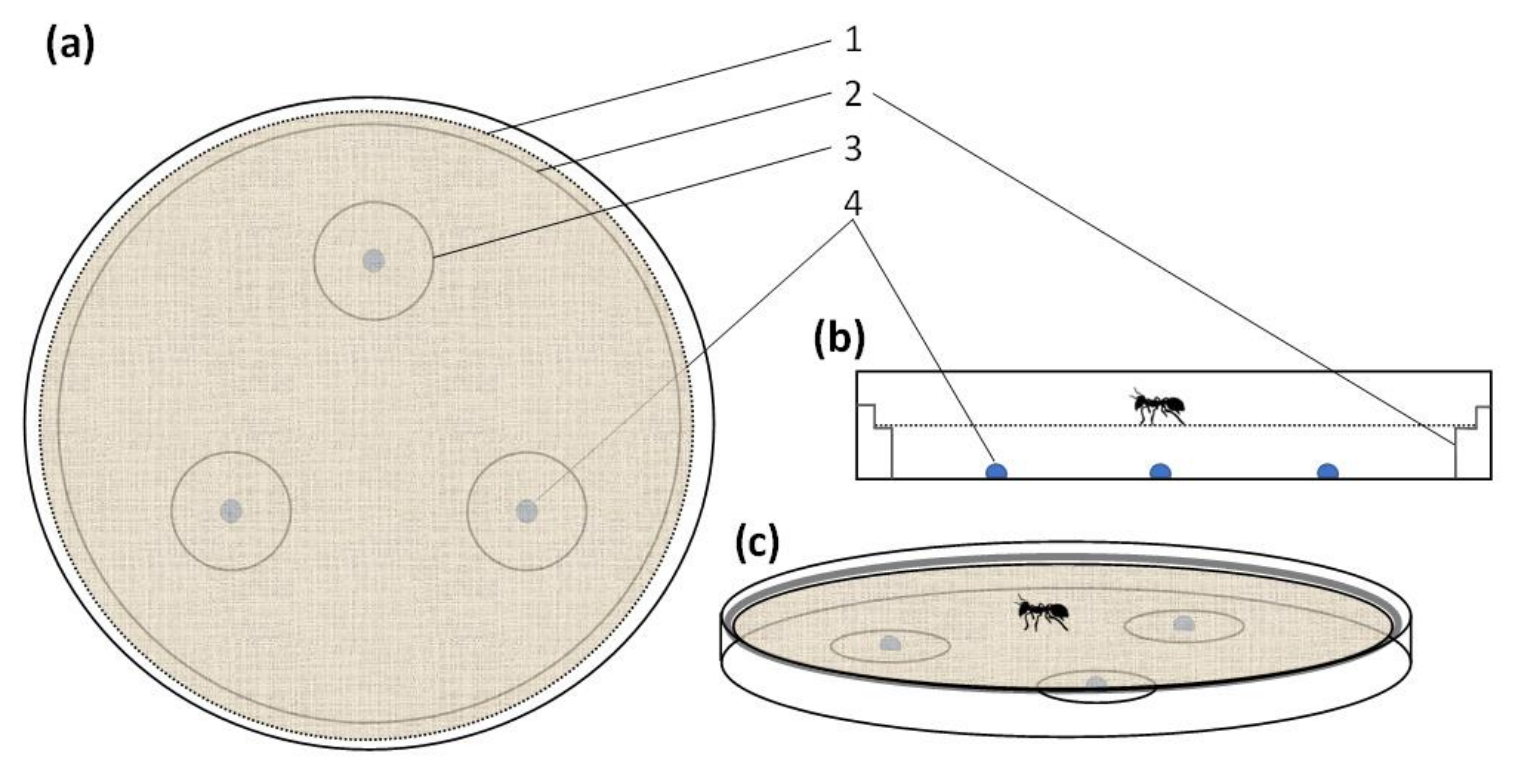

Figure 8.1 Ant bioassay design to test ant attraction based on volatile headspace of nectar droplets without contact. View of $9 \mathrm{~cm}$ petri dish from above (a), side view (b), and at an angle (c). The steel mesh (1) is supported by a ledge on the plastic ring (2) that fits inside the petri dish perimeter. The $18 \mathrm{~mm}$ ring (3) marked on the bottom of the petri dish encircled the placement of $2 \mu \mathrm{L}$ EFN, sucrose, or water droplets (4). This zone was used to quantify time ants spent within the "headspace zone" of each droplet. 


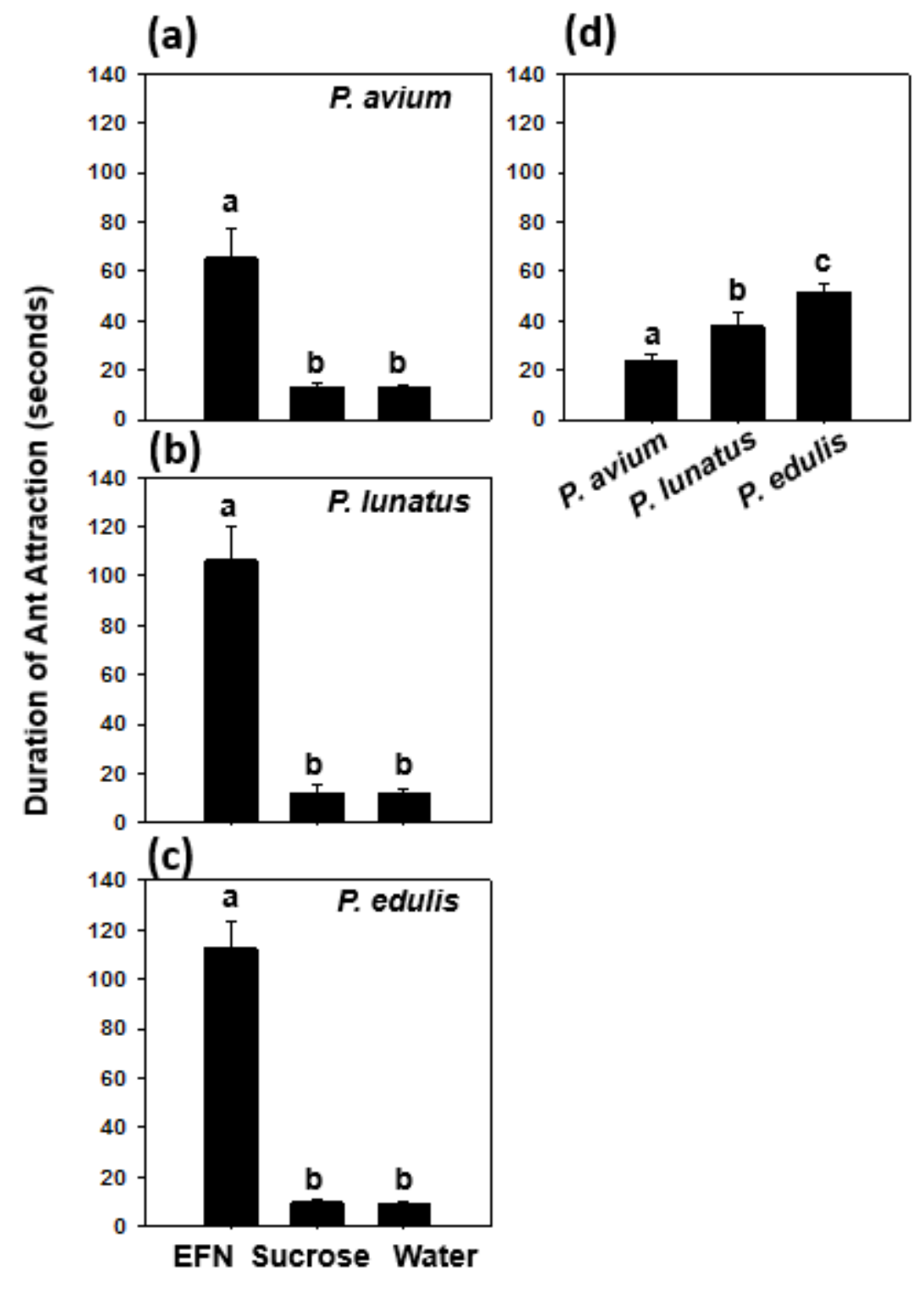

Figure 8.2 Ants distinguish EFN from controls without contact. One-way ANOVAs and Holm-Sidak post hoc analyses confirm ants' ability to sense and distinguish EFN from aqueous sucrose and water controls (a-c), as well as EFNs from each plant species, Prunus avium, Phaseolus lunatus, and Passiflora edulis (d), all from the airspace above each droplet. Letters indicate significant differences in mean time spent within each zone $\left(F_{2,29}=\right.$ $12.447, p<0.001)$, error bars represent standard error of the mean $(n=10$ ants for each of the four trials). 


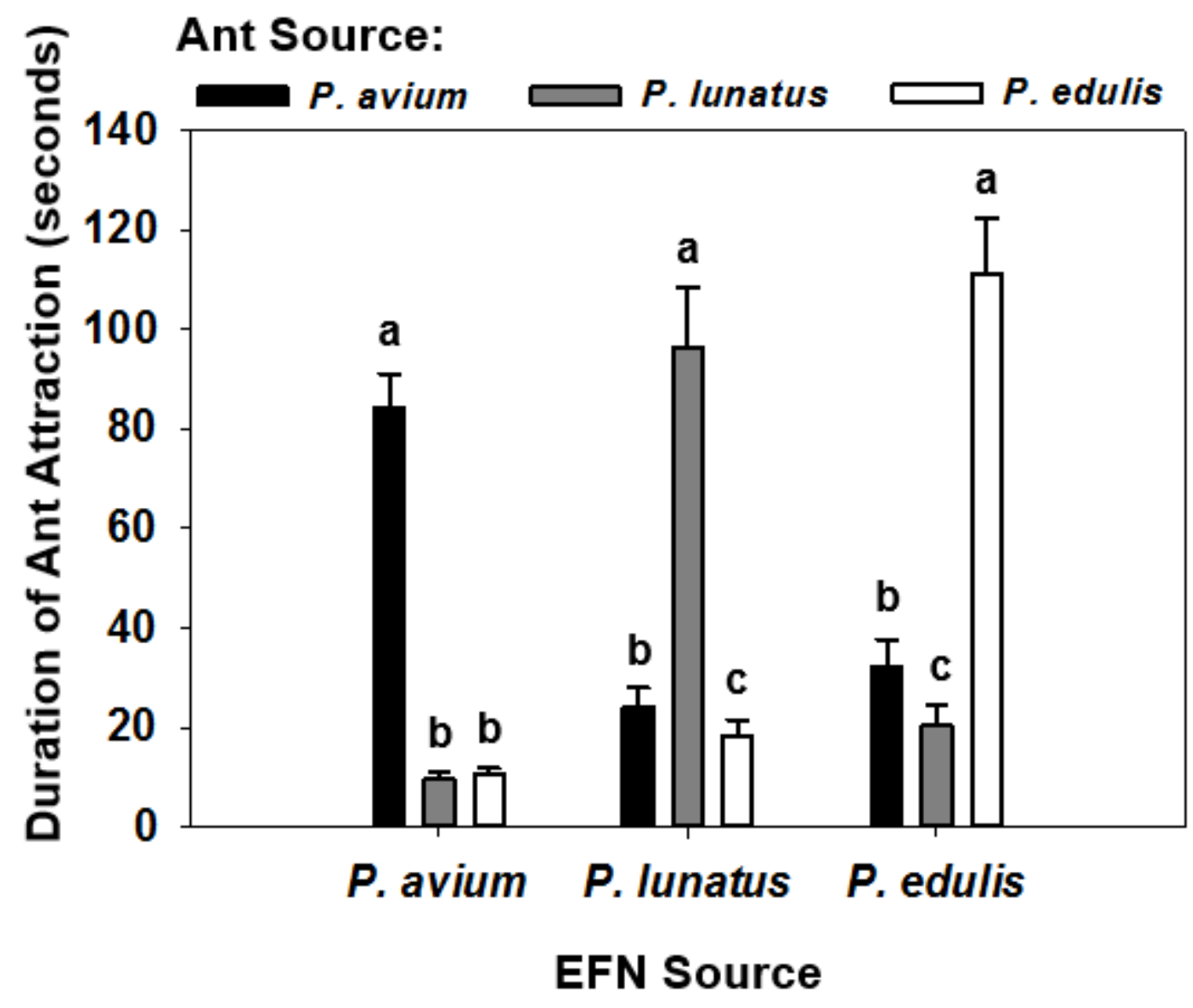

Figure 8.3 Ants choose host plant EFN based on scent. Host plants from which ants were collected produce the most attractive VOC-sphere. EFN collection source for each trial shown on the x-axis; bar color shows plant species from which Lasius niger ants were collected: $P$. avium (black bars), $P$. lunatus (gray bars), and P. edulis (white bars). Letters indicate significant differences from one-way ANOVA tests and Holm-Sidak post hoc analyses for mean time ants collected from each host plant spent in each nectar zone $(P$. avium ants: $F_{2,29}=24.382, p<0.001, P$. lunatus ants: $F_{2,29}=45.759, p<0.001 ; P$. edulis ants: $\left.F_{2,29}=74.514, p<0.001\right)$. Error bars represent standard error of the mean ( $n=10$ ants per trial, figure displays data from three separate trials). 


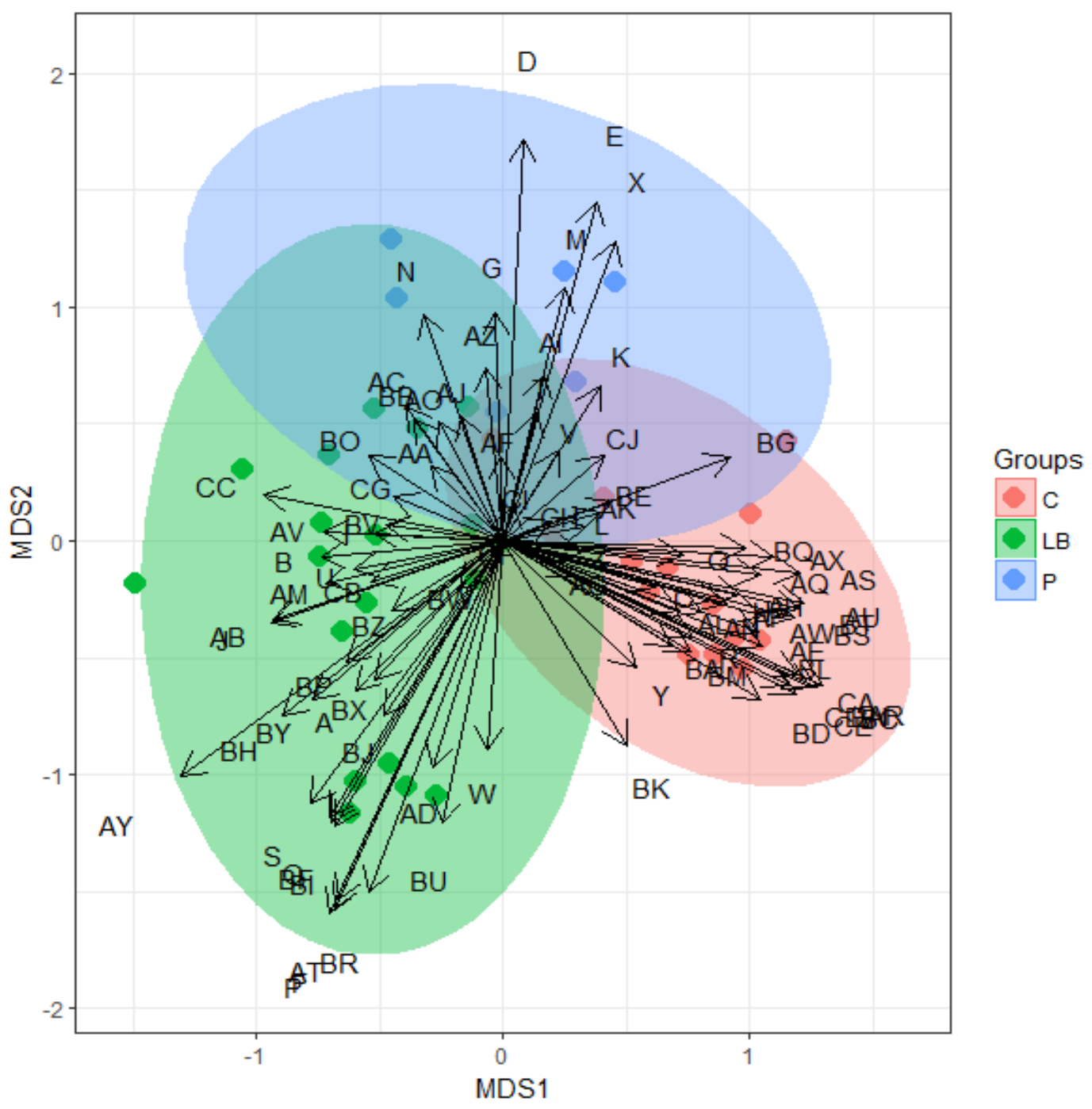

Figure 8.4 Suites of EFN volatiles vary by plant species. Multidimensional scaling (MDS) plot of each EFN sample calculated from Bray-Curtis distances using TIC peak areas of all 87 compounds. EFN volatile scent profiles from $P$. avium, $P$. lunatus, and $P$. edulis reveals significant species-specific differences in suites of VOCs (MDS stress value $=0.236$, ANOSIM: $R=0.6003$, $p<0.001)$. Each point represents a separate EFN sample. Plant species is represented by color of point and ellipse showing $95 \%$ confidence; $P$. avium, or cherry, $n=14$ (red), P. lunatus, lima bean, $n=19$ (green), and P. edulis, passionflower, $n=6$ (blue). MDS plot was made using ggord and vegan packages in $R$. 


\section{Chapter 9: Conclusions and Future Directions}

Plant families able to form a symbiosis with nitrogen-fixing bacteria, such as legumes with rhizobia, are relatively rare, but occur in most terrestrial ecosystems. Legume-rhizobia symbioses have been long-recognized to play critical roles in geochemical cycling and plant productivity, acclaimed as ecosystem engineers and keystone species (Wardle et al. 2002). Gaining organic nitrogen $(\mathrm{N})$ in exchange for photo-assimilated carbon $(\mathrm{C})$ aids plants in overcoming soil nitrogen limitations, relevant both ecologically and agriculturally. The work I present here indicates that symbiotic resource exchange influences food webs beyond plant productivity; rhizobia also influence aboveground food webs from the bottom-up by mediating plant defense chemistry and tritrophic interactions.

One central finding in my dissertation is the difference between rhizobia-mediated direct and indirect defenses. Rhizobia increase lima bean cyanogenic potential, a N-based direct defense most effective against chewing herbivores (Thamer et al. 2011, Godschalx et al. 2015), but decrease the predatory ant and parasitoid wasp recruitment essential to the efficacy of Crich indirect defense traits: extrafloral nectar $(\mathrm{EFN})$ and volatile signal production (volatile organic compounds; VOC), both of which are reduced in rhizobia-inoculated plants (Godschalx et al. 2015), Godschalx et. al, under 
review). The next question these data present is: what mechanism(s) explain the rhizobia-mediated differences in plant trait allocation and consequential aboveground interactions? Bottom up effects of beneficial microbes are context dependent in their influence on plant-herbivore and plant-predator interactions. Understanding tritrophic responses to symbiotic microbes requires a more detailed mechanism driving the changes in plant chemical biology. Here, I propose two hypotheses.

The first hypothesis I propose is a "carbon sink" hypothesis, which explains rhizobia-mediated plant defense traits by the ratio of available resources; $\mathrm{N}$ is relatively more abundant with a $\mathrm{N}$-fixing symbiont, while microbial metabolism demands may limit available $\mathrm{C}$. The carbon-sink hypothesis assumes microbes acting as a strong $\mathrm{C}$ sink limit plants from being able produce the $\mathrm{C}$-rich signals or rewards that effectively recruit predators, and that by provisioning $\mathrm{N}$, plants can more readily produce $\mathrm{N}$ demanding traits. The carbon-sink hypothesis inherits elements of the carbon-nutrient balance hypothesis—-such as the view that plants adjust trait allocation to passively match internal ratios of available C and N. Framework for the carbon-sink hypothesis is also inherited from the growthdifferentiation balance hypothesis (Herms and Mattson 1992, Stamp 2003), considering symbiotic cooperation as "differentiation" to extend the "grow versus defend" paradigm to include "grow versus defend versus cooperate". 
The second hypothesis I propose is my "functional-shift" hypothesis, which explains rhizobia-mediated differences in defense allocation as the plant phenotype conferring greatest fitness in the herbivore- and predatorcommunity context. Framework contributing to the functional shift hypothesis includes optimal defense hypothesis (McKey 1974, Cates and Rhoades 1977). which posits that patterns of defense allocation should be explained by maximized fitness. Inducibility of defenses to conserve resources, and distributing defenses within-plant to the most beneficial organs for defense both arose within the optimal defense hypothesis. Similarly, if plants with microbes effectively defend using direct defense strategies, indirect defenses may be excessive resource cost and less reliable, depending on animal density and behavior. The functional-shift hypothesis I propose here assumes symbiotic formation leads to suites of traits co-varying as microbially mediated defense syndromes.

Here I will discuss the implications of my data and relevant literature in the light of my two working hypotheses: the "carbon sink hypothesis", and the "functional shift hypothesis" to explain mechanisms underlying how rhizobia and other belowground microbial symbionts influence their host plants' aboveground interactions. Carbon sink hypothesis 
Microbial symbiont metabolism plays a largely underestimated role in terrestrial carbon cycles as sinks for organic carbon (Pringle 2015). Indeed, rhizobia nodules can consume up to $16-30 \%$ of the plant's total photosynthate (Peoples et al. 1986, Kaschuk et al. 2009). If the C committed to rhizobia is the limiting factor preventing plants from C-rich secondary metabolism, we would expect to see a decrease in C-demanding traits.

C-rich plant traits, such as EFN have been considered to be relatively "cheap" compared with $\mathrm{N}$-demanding traits, however, in reality, certain traits may not be as cheap as previously thought. Removing photosynthetic area quantitatively reduces the amount of EFN plants produce, implying carbon limitations (Ballhorn et al. 2014b). Carbohydrates secreted in EFN may be recoverable for plant repurposing, but the resorption mechanisms characterized for floral nectars (Nepi and Stpiczyńska 2007) have not been established for EFN, implying a potential loss of resources secreted (Heil 2011). Plant EFN secretion patterns further indicate an investment cost, as EFN is typically induced only when plants are under attack. Plants produce more nectaries or secrete more nectar from plant organs most important for fitness (Radhika et al. 2008, Godschalx et al. 2015, 2016), and can invest defensive toxins to prevent over excessive consumption. Further, in my dissertation I show EFN volatiles elicit strong ant behavioral responses, indicating a plant investment into a well-advertised resource (Chapter 8) 
(Godschalx et. al, in prep). Supporting the carbon sink hypothesis, rhizobiainoculated plants secrete less EFN, resulting in lower ant attraction than rhizobia-free conspecifics (Godschalx et al>, 2015b) (Chapter 7).

Rhizobia-inoculated lima bean plants also attract fewer parasitoid wasps via herbivore-induced VOCs (Chapter 6; Godschalx et. al, under review). Volatile compounds are another unrecoverable $\mathrm{C}$ sink lost to atmospheric processes. Producing a 5-carbon isoprene unit, the basic unit for other, more complex terpenoids, requires 6 carbons, 20 ATP and 14 NADPH via the more efficient non-Mevalonate (methylerythritol phosphate MEP) pathway, or 9 carbons, $24 \mathrm{ATP}$ and $14 \mathrm{NADPH}$ via the Mevalonate (MVA) pathway (Sharkey and Yeh 2001), implying significant physiological costs to producing an entire suite of VOCs. VOC release is influenced belowground microbial symbionts. Plants show reduced sesquiterpene production in response to herbivory when associating with arbuscular mycorrhizal fungi (Fontana et al. 2009). Compromised volatile emission in lima bean with rhizobia supports the carbon sink hypothesis as rhizobia-inoculated plants released overall fewer total volatiles, and reduced amounts of all VOCs apart from a few exceptions (Ballhorn et al. 2013).

If resource constraints can explain rhizobia-mediated defense allocation, an increase in $\mathrm{N}$-demanding defenses would also be expected to emerge in plants receiving fixed $\mathrm{N}$ from symbionts. This rationale may 
explain why one of the only volatiles in the above example that increase with $\mathrm{N}$-fixing rhizobia is indole, a $\mathrm{N}$-containing volatile (Ballhorn et al>, 2013c). Other $\mathrm{N}$-demanding traits involving proteinogenic amino acids found in higher concentrations in rhizobia-inoculated plants include direct defenses, trypsin protease inhibitors (Godschalx, unpublished data), and cyanogenesis (Thamer et al. 2011, Godschalx et al. 2015). In fact, my dissertation shows the genotypic relationship between cyanogenesis and nodule formation is quantitative. High cyanotypes (HC) consistently form greater numbers of nodules than low cyanotypes (LC) (Chapter 5; Godschalx et. al, under review). Such discrepancy in nodulation putatively creates a stronger C sink, based on sink strength increases in plants with interacting belowground symbionts (Harris et al., 1985; Wright et al., 1998; Jia \& Gray, 2008). HC plants, with hundreds more nodules than LC plants, show constrained primary metabolism through the negative relationship between nodule number and foliar protein, implying that while $\mathrm{N}$-provisioning may have contributed to cyanotype evolution, the $\mathrm{C}$ sink that emerged may not be adaptive, although this would need to be tested.

A few ways to test the carbon sink hypothesis could be through direct tests of plant carbon dynamics or as a targeted meta-analysis. Directly testing carbon constraints often involve photosynthetic area removal (Ballhorn et al. 2014b). Examining interactions between rhizobia and 
defoliation on carbon-demanding plant traits such as extrafloral nectar, could provide some insight into the degree to which plants are photosynthatelimited. A more analytical approach could involve testing ${ }^{12} \mathrm{C}$ : ${ }^{13} \mathrm{C}$ isotope ratios in EFN. Plants that express higher ${ }^{13} \mathrm{C}$ content are likely stretching their photosynthetic potential, using as much $\mathrm{CO}_{2}$ as possible. While it is well-established that microbes, including $\mathrm{N}$-fixing rhizobia, stimulate photosynthetic rates by creating a deficit (sink stimunlation) (Harris et al>, 1985; Jia \& Gray, 2008; Kaschuk>et al>, 2009; Pringle, 2015b), the question of whether the photosynthetic capacity of the plant is reaching limits that would constrain defense allocation has yet to be answered. Finally, a metaanalysis of studies quantifying the carbon flow dynamics of plant-microbe interactions across different classes, (AMF, ectomycorrhizae, N-fixing rhizobacteria, nodulating $\mathrm{N}$-fixing rhizobia/Frankia) may be useful to examine carbon-costs of various symbiont associations.

\section{Functional-shift hypothesis}

An alternative hypothesis to resource ratios modulating plant defense allocation, is the potential for symbionts to influence plant success and select for an entire defense syndrome. If plant phenotype with rhizobia tend to enable greater plant fitness via allocation into direct defenses versus indirect defenses, we would expect for plant trait allocation to follow fitness benefits. From the time endosymbiosis forms and begins exchanging signals and 
resources to the end of the symbiotic lifespan resulting in nodule senescence, many different regulatory pathways are active and plants receive many chemical stimuli that could influence other developmental patterns (Atkins et al., 1984; van Brussel et al., 2002; Kiers et al., 2003; Sun et al., 2006; Cooper, 2007). Can symbiosis trigger a plant response that creates a regulatory cascade driving a strategic shift in defensive strategy? If rhizobia-colonized plants are partially released from herbivory via cyanogenesis, herbivore feeding guilds that reduce plant fitness most drastically would be targeted by rhizobia-mediated defense chemistry. Subsequently, if predators and parasitoid wasp foraging is less efficient on rhizobia-inoculated plants due to compromised VOC production or higher trichome density, this could suggest a tradeoff in entire defense syndromes. Rhizobia-mediated tritrophic interactions may provide plants with a greater overall advantage by increasing direct defense and reducing indirect defense, causing a shift in functional defensive strategy.

Instead of the ratio of resources available passively determining what plants will produce, resource, both $\mathrm{C}$ and $\mathrm{N}$, are often allocated independently from their availability, indicating plants can actively prioritize trait investment. Even before considering microbial effects, defense allocation in nature does not necessarily reflect nutrient availability. Despite having greater amounts of foliar nitrogen, Brassica oleracea plants fertilized with synthetic compared 
with organic soil amendments produce lower concentrations of the defensive glucosinolates, glucobrassicin and sinigrin (Staley et al. 2010). Throughout a time series trial, both $\mathrm{N}$ content and each glucosinolate are higher in plants with lower levels of either fertilizer type. Increasing $\mathrm{N}$ availability does increase isoprene emission in velvet bean, but the percentage of $\mathrm{C}$ released as isoprene decreases across the increasing $\mathrm{N}$ gradient, supporting the notion that carbon limitations may not be restricting volatile production (Harley et al. 1994). Total inducible VOC production quantitatively increases across demineralized, half, and complete nutrient treatments, however, not all compounds follow this response to nutrient availability. Certain compounds including indole and the monoterpene, geranyl acetate, increase with $\mathrm{N}$, yet another monoterpene, linalool, and several sesquiterpenes are induced in levels independent from nutrient availability (Gouinguené and Turlings 2002). The same sesquiterpene, a-bergoamotene, along with limonene and germacrene A released by Nicotiana attenuata are unaffected by high and low nitrogen treatments (Lou and Baldwin 2004). High and low nitrogen treatments in this example also produce mixed effects on direct defensive compounds that are $\mathrm{N}$-demanding, such as nicotine and trypsin inhibitors, or C-demanding: chlorogenic acid and diterpene glycosides. Plant defense hormonal signals respond differently to nitrogen availability. Jasmonic acid (JA) and salicylic acid (SA) bursts when induced under low nitrogen were 
$39 \%$ and $60 \%$ of the responses observed under high nitrogen, implying low nitrogen limited JA burst more strongly than it limited the SA burst (Lou \& Baldwin, 2004). In addition to the indirect and direct defense examples presented here that do not correlate to $\mathrm{N}$ or $\mathrm{C}$ availability, plants can tolerate varying degrees of herbivory, which also does not correlate in direct response to resource availability. In a meta-analysis, 17 out of 24 studies show plants with low nutrients are more tolerant to herbivory than conspecifics with higher nutrients (Wise \& Abrahamson, 2007). Taken together, plant defense allocation can act independently from nitrogen pools.

If plant defense production does not parallel the nutrients afforded to the plant, nitrogen provided by microbes also may not directly determine defense levels. A few interesting distinctions between soil-acquired and microbial-provisioned nitrogen include i) the molecular form of nitrogen provided, ii) the potential for the source to be more reliable on a longer term, and iii) systemic responses to the infection. Lima bean transports ureides when relying on nitrogen fixation, but with increasing concentrations of exogenous nitrate, the xylem is increasingly dominated by nitrates and amides (Peoples 1987). C and N metabolism of nodules are closely linked in a metabolic cycle that requires plants to also provide $\mathrm{N}$ to rhizobia. Plantderived branched-chain amino acids along with carbohydrates enable rhizobia to cycle amino acids back to the plant, including Leu, Ile, and Val 
(Lodwig et al., 2003; Prell et al., 2009). Cyanogenic glucosides in lima bean are derived from Ile or Val, indicating a potential interaction between defensive chemistry and symbiotic regulation (Møller 2010). The presence or absence of a defensive compound can depend nodulation as well: plant alkaloid synthesis depends on enzymes for early stages of biosynthesis found only in nodule tissue (Irmer et al. 2015). Rhizobia can also alter within-plant allocation patterns. Compared with $\mathrm{CaNO}_{3}$-provisioned plants, rhizobiainoculated plants produce similar cyanogenic potential in the youngest leaves, but maintain high cyanogenic potential in older leaf stages, which drops off in $\mathrm{N}$-fed plants. The result is rhizobial plants with cyanogenesis distributed throughout the entire plant versus only in the youngest leaves (Godschalx, unpublished data). Such patterns may imply plants allocate N from nodules in pattern that reflect a more sustained source. Cues to indicate the sustainability of rhizobia as an $\mathrm{N}$ source may be derived from the regulation and persistence of this infection. Systemic responses cascade through the plant in response to microbial infection, and beneficial microbes are no exception. Molecular patterns interact in multifactorial experiments showing transcriptional patterns with AMF, rhizobia, or the combination of both (Afkhami and Stinchcombe 2016). Plants exposed to foliar pathogen infection form a reduced number of nodules, which, although this remains to be confirmed, is likely mediated by the systemic increase in antipathogen 
polyphenol oxidases observed in both below- and aboveground tissues (Ballhorn et al. 2014a). Taken together, plants engaging in belowground symbioses have a physiological state different from non-associating conspecifics with equivalent nutrient status.

To test the functional shift hypothesis that rhizobia symbiosis triggers changes in plant defense syndromes, the effects of rhizobia-inoculated (R+) versus rhizobia-free (R-) treatments on plant fitness under a series of manipulated tritrophic treatments would be interesting. Indirect defense responses to belowground symbionts are context-dependent, which may largely be due to microbial identity: rhizobia versus soil rhizobacteria versus mycorrhizal fungi (reviewed in Schädler and Ballhorn 2016, Rasmann et al. 2017).

Bottom-up effects from rhizobia may unevenly influence plant local food webs depending on feeding guild and specialization. Different feeding guilds trigger SA versus JA defensive pathways; specialists versus generalists would be affected differently by rhizobia-mediated plant chemistry. Predator fitness also depends on foraging ability and host quality. Whether parasitoid fitness increases or decreases on hosts raised on rhizobial plant tissue is not known, however, soil rhizobacteria interfere with foraging ability of Diaeretiella rapae by altering the JA-specific signal used for attraction (Pineda et al. 2013). Generalist aphids, Myzus persicae, grow bigger on 
growth-promoting soil rhizobacteria-treated plants (Pineda et al. 2012), yet, in this system, fewer parasitoids are attracted to rhizobacteria-inoculated plants than to rhizobacteria-free plants (Pineda et al. 2013), consistent with my findings for lima bean in the field from Chapter 6, in which rhizobiainoculation resulted in lower parasitoid attraction (Godschalx et. al, under review). Similar to the JA interruption observed in the A. thalianarhizobacteria system, $\mathrm{R}+$ lima bean plants produce fewer volatiles overall, including many JA-associated cues, but increase shikimate products, indole and methyl salicylate (Ballhorn et al. 2013). Increasing dependency of soybean plants on rhizobia (versus nitrate) for nutrition forms a positive relationship with SA accumulation, but a negative trend with accumulation of both cis- and trans- JA, although trends were not significant (Dean et al. 2014). SA-induced molecular patterns in A. thaliana with both rhizobacteria and aphids initially increased and then tapered off, potentially indicating a reliable (repellant in this case) cue. (Pineda et al. 2012). Such repellency makes sense if hosts are less likely to be present on rhizobial-associated plants. Soybean rhizobia reduce aphid colonization, even on a quantitative level; aphids per plant form a negative correlation with nodule number.

(Dean et al. 2009, Brunner et al. 2015)

Chewing herbivores (Helicoverpa zea) have increased growth on plants nutritionally relying on rhizobia, although prefer nitrate-fed leaves over 
rhizobia treatments in cafeteria trials. (Dean et al. 2014). However, volatiles induced by chewing herbivores are attenuated in rhizobacteria-inoculated $A$. thaliana plants. These volatiles included methyl salicylate, and notably, abergamotene, which is unaffected by plant nitrogen status (Gouinguene \& Turlings, 2002). Interestingly, parasitoid Microplitis mediator distinguishes between chewing herbivore-infested plants with and without soil rhizobacteria, and is more attracted to uninoculated plants (Pangesti et al. 2015), the opposite response from that of the aphid parasitoid (Pineda et al. 2013). Such response may be adaptive because the chewing herbivore has reduced growth on rhizobacteria-mediated plants, which, compared with rhizobacteria-free plants also have increased constitutive and chewingherbivore-induced aliphatic glucosinolates (Pangesti et al. 2016). The role of specialization may affect the specialist cabbage aphid and parasitoid to consume glucosinolate-rich food, and should be investigated further. Specialist herbivores for lima bean show reduced growth on highly cyanogenic lima bean tissues, and preference for R- plants (Ballhorn et al>, 2007, 2013c). Cyanogenic strains of Trifolium repens reduced herbivore growth with rhizobia, but in acyanogenic strains, rhizobia conferred increased herbivore nutrition (Kempel et al. 2009).

Overall, it seems defensive responses of plants with rhizobia negatively influence herbivores from multiple feeding guilds more strongly 
through direct defense rather than fear of impending attack since parasitoid preferences discovered thus far reflect the same plants preferred by herbivores, which are R- plants for aphids (Dean et al. 2009, Brunner et al. 2015), and chewing herbivores (Ballhorn et al. 2013, Dean et al. 2014). Evidence for direct defenses resulting from rhizobia inoculation that could explain herbivore and consequently predator attraction away from $\mathrm{R}+$ plants include potential nutritional effects, since with increasing nodules, lima beans leaf tissue contains quantitatively higher hydrogen cyanide per protein (Chapter 5; Godschalx et. al, under review). The association between cyanotype and significant differences in nodule formation is particularly interesting given that nitrogen status does not increase cyanogenic glycoside formation (Forslund and Jonsson 1997). Although the role of cyanogenic glycosides in mobilizing nitrogen throughout the plant has been suggested for primary metabolism or symbiotic regulation (Selmar et al. 1988, Møller 2010). If foliar nitrogen from symbionts can be locked away in an herbivoreinaccessible form, harmful even to specialists (Ballhorn et al. 2007), and biomass increases associated with belowground symbionts potentially reduces predator foraging (Gange et al. 2003), direct defenses seem to confer plants engaging in symbiosis a greater benefit.

When is investing in traits to recruit predators more effective than investing in defensive chemistry? Do rhizobia exert a stronger explanatory 
force driving patterns of resource allocation than does strict resource availability? The mechanisms underlying why rhizobia and other belowground microbial symbionts influence their host plants' aboveground interactions remain to be fully understood. The two hypotheses I propose here aim to address recent calls to integrate existing theory with new knowledge about how symbiotic cooperation shapes defense investments (Heath et al. 2014). Context-dependent patterns in plant defense are typically discussed within a framework established by several hypotheses predicting defense allocation across a gradient of nutrient availability (Herms and Mattson 1992). However, nutrient acquisition is one of the many challenges to the sessile lifestyle that many plants overcame by cooperating with other, often more mobile organisms. Symbionts that spread gametes, disperse seeds, evict herbivores, or provide organic forms of mineral nutrients radically alter plant resource allocation dynamics. Through the carbon sink hypothesis, resource cost and benefit economics of symbiotic relationships determine resource use for other growth and defense traits (Werner et al., 2014). According to the functional shift hypothesis, plant fitness resulting from collective interactions with the communities of herbivores, predators, pollinators, and seed-dispersers sculpt plant defense syndromes. The role of symbionts affecting plant fitness via altered trophic responses that consequently shape defensive syndromes has not previously been tested. 
Virtually all plants rely on mutualists to provide an ecological service (in varying degrees of obligation). Exploring the mechanisms by which symbionts alter food web pressure on plant survival feeds back to aid in our understanding of the persistence of beneficial relationships-in the context of plant antagonists. Understanding symbiotic relationships brings us closer to developing sustainable solutions for pest management and grasping community-level food web dynamics through a functional ecology approach to plant defense. 


\section{Literature Cited}

Adler LS, Seifert MG, Wink M, Morse GE. 2012. Reliance on pollinators predicts defensive chemistry across tobacco species. Ecology letters 15: 11408.

Afkhami ME, Rudgers JA, Stachowitcz JJ. 2014. Multiple mutualism effects: conflict and synergy in multispecies mutualisms. Ecology 95: 833844.

Agrawal AA. 2007. Macroevolution of plant defense strategies. Trends in ecology \& evolution 22: 103-9.

Agrawal AA. 2011. Current trends in the evolutionary ecology of plant defence. Functional Ecology 25: 420-432.

Agrawal AA, Conner JK, Rasmann S. 2010. Tradeoffs and negative correlations in evolutionary ecology. M.A. Bell, W.F. Eanes, D.J. Futuyma, and J.S. Levinton, eds. Evolution After Darwin: the First 150 Years. Sinauer Associates, Sunderland,.

Agrawal AA, Fishbein M. 2006. Plant defense syndromes. Ecology 87: S132-S149.

Alba C, Bowers MD, Hufbauer R. 2012. Combining optimal defense theory 
and the evolutionary dilemma model to refine predictions regarding plant invasion. Ecology 93: 1912-1921.

\begin{abstract}
Algar E, Gutierrez-Mañero FJ, Garcia-Villaraco A, García-Seco D, Lucas JA, Ramos-Solano B. 2014. The role of isoflavone metabolism in plant protection depends on the rhizobacterial MAMP that triggers systemic resistance against Xanthomonas axonopodis pv. glycines in Glycine max (L.) Merr. cv. Osumi. Plant Physiology and Biochemistry: 1-8.
\end{abstract}

Arenas A, Farina WM. 2012. Learned olfactory cues affect pollen-foraging preferences in honeybees, Apis mellifera. Animal Behaviour 83: 1023-1033.

Assunção M a., Torezan-Silingardi HM, Del-Claro K. 2014. Do ant visitors to extrafloral nectaries of plants repel pollinators and cause an indirect cost of mutualism? Flora - Morphology, Distribution, Functional Ecology of Plants 209: 244-249.

Atkins CA, Shelp BJ, Kuo J, Peoples MB, Pate JS. 1984. Nitrogen nutrition and the development and senescence of nodules on cowpea seedlings. Planta 162: 316-326.

Atsatt PR, O'Dowd DJ. 1976. Plant defense guilds. Science 193: 24-29.

Baldwin IT, Hamilton W. 2000. Jasmonate-induced responses of Nicotiana sylvestris results in fitness costs due to impaired competitve ability for 
nitrogen. Journal of Chemical Ecology 26: 915-952.

Baldwin IT., Sims CL, Kean SE. 1990. The reproductive consequences associated with inducible alkaloidal responses in wild tobacco. Ecology 71: $252-262$.

Ballhorn DJ. 2011a. Cyanogenic glycosides in nuts and seeds. In: Preedy VR,, In: Watson RR,, In: Patel VB, eds. Nuts \& Seeds in Health and Disease Prevention. Elsevier Inc., 129-136.

Ballhorn DJ. 2011b. Constraints of simultaneous resistance to a fungal pathogen and an insect herbivore in lima bean (Phaseolus lunatus L.). Journal of Chemical Ecology 37: 141-144.

Ballhorn DJ, Godschalx AL, Kautz S. 2013a. Co-variation of chemical and mechanical defenses in lima bean (Phaseolus lunatus L.). Journal of Chemical Ecology 39: 413-417.

Ballhorn DJ, Godschalx AL, Smart SM, Kautz S, Schädler M. 2014a. Chemical defense lowers plant competitiveness. Oecologia 176: 811-824.

Ballhorn DJ, Heil M, Lieberei R. 2006. Phenotypic plasticity of cyanogenesis in lima bean Phaseolus lunatus-activity and activation of betaglucosidase. Journal of Chemical Ecology 32: 261-275. 
Ballhorn DJ, Heil M, Pietrowski A, Lieberei R. 2007. Quantitative effects of cyanogenesis on an adapted herbivore. Journal of Chemical Ecology 33: 2195-2208.

Ballhorn DJ, Kautz S, Heil M. 2013b. Distance and Sex Determine Host Plant Choice by Herbivorous Beetles. 8.

Ballhorn DJ, Kautz S, Heil M, Hegeman AD. 2009a. Cyanogenesis of wild lima bean (Phaseolus lunatus L .) is an efficient direct defence in nature. PloS One 4: 1-7.

Ballhorn DJ, Kautz S, Lieberei R. 2010a. Comparing responses of generalist and specialist herbivores to various cyanogenic plant features. Entomologia Experimentalis et Applicata 134: 245-259.

Ballhorn DJ, Kautz S, Lion U, Heil M. 2008a. Quantitative variability of lima bean's VOC boquets and its putative ecological consequences. Plant Signaling and Behavior 3: 1005-1007.

Ballhorn DJ, Kautz S, Lion U, Heil M. 2008b. Trade-offs between direct and indirect defences of lima bean (Phaseolus lunatus). Journal of Ecology 96: 971-980.

Ballhorn DJ, Kautz S, Rakotoarivelo FP. 2009b. Quantitative variability of cyanogenesis in Cathariostachys madagascariensis-the main food plant of 
bamboo lemurs in Southeastern Madagascar. American Journal of Primatology 71: 305-315.

Ballhorn DJ, Kautz S, Schädler M. 2013c. Induced plant defense via volatile production is dependent on rhizobial symbiosis. Oecologia 172: 833846.

Ballhorn DJ, Kay J, Kautz S. 2014b. Quantitative effects of leaf area removal on indirect defense of lima bean (Phaseolus lunatus) in nature. Journal of Chemical Ecology 40: 294-296.

Ballhorn DJ, Lieberei R. 2006. Oviposition choice of Mexican bean beetle (Epilachna varivestis) depends on host plants cyanogenic capacity. Journal of chemical ecology 32: 1861-5.

Ballhorn DJ, Lieberei R, Ganzhorn JU. 2005. Plant cyanogenesis of Phaseolus lunatus and its relevance for herbivore-plant interaction: the importance of quantitative data. Journal of Chemical Ecology 31: 1445-1473.

Ballhorn DJ, Pietrowski A, Lieberei R. 2010b. Direct trade-off between cyanogenesis and resistance to a fungal pathogen in lima bean (Phaseolus lunatus L.). Journal of Ecology 98: 226-236.

Ballhorn DJ, Schiwy S, Jensen M, Heil M. 2008c. Quantitative variability of direct chemical defense in primary and secondary leaves of lima 
bean (Phaseolus lunatus) and consequences for a natural herbivore. Journal of Chemical Ecology 34: 1298-1301.

Ballhorn DJ, Younginger BS, Kautz S. 2014c. An aboveground pathogen inhibits belowground rhizobia and arbuscular mycorrhizal fungi in Phaseolus vulgaris. BMC Plant Biology 14: 1-13.

Barto EK, Cipollini D. 2005a. Testing the optimal defense theory and the growth-differentiation balance hypothesis in Arabidopsis thaliana. Oecologia 146: $169-178$.

Barto EK, Cipollini D. 2005b. Testing the optimal defense theory and the growth-differentiation balance hypothesis in Arabidopsis thaliana. Oecologia 146: $169-178$.

Barton KE. 2007. Early ontogenetic patterns in chemical defense in Plantago (Plantaginaceae): genetic variation and trade-offs. American Journal of Botany 94: 56-66.

Bennett AE, Bever JD, Deane Bowers M. 2009. Arbuscular mycorrhizal fungal species suppress inducible plant responses and alter defensive strategies following herbivory. Oecologia 160: 771-9.

Bernays E, Graham M. 1988. On the evolution of host specificity in phytophagous arthropods. Ecology 69: 886-892. 


\section{Birkett MA, Campbell CA, Chamberlain K, Guerrieri E, Hick AJ,}

Martin JL, Matthes M, Napier JA, Pettersson J, Pickett JA, et al. 2000. New roles for cis-jasmone as an insect semiochemical and in plant defense. Proceedings of the National Academy of Sciences of the United States of America 97: 9329-9334.

Bixenmann RJ, Coley PD, Kursar TA. 2013. Developmental changes in direct and indirect defenses in the young leaves of the neotropical tree genus Inga (Fabaceae). Biotropica 45: 175-184.

Bonaventure G. 2012. Perception of insect feeding by plants. Plant Biology 14: $872-880$.

Bradford M. 1976. A rapid and sensitive method for the quantitation of microgram quantities of protein utilizing the principle of protein-dye binding. Analytical Biochemistry 72: 248-254.

Briggs MA. 1990. Chemical defense production in Lotus corniculatus L. I. The effects of nitrogen source on growth, reproduction and defense. Oecologia 83: $27-31$.

Broadway RM, Duffey SS. 1986. Plant proteinase inhibitors: Mechanism of action and effect on the growth and digestive physiology of larval Heliothis zea and Spodoptera exiqua. Journal of Insect Physiology 32: 827-833. 
Brockwell J, Bottomley PJ, Thies JE. 1995. Manipulation of rhizobia microflora for improving legume productivity and soil fertility: A critical assessment. Plant and Soil 174: 143-180.

Bronstein JL. 2001. The costs of mutualism. American Zoologist 41: 825839.

Brouat C, McKey D, Bessière J-M, Pascal L, Hossaert-McKey M. 2000. Leaf volatile compounds and the distribution of ant patrollingin an ant-plant protection mutualism: Preliminary results on Leonardoxa (Fabaceae: Caesalpinioideae) and Petalomyrmex (Formicidae: Formicinae). Acta Oecologica 21: 349-357.

Brown PD, Tokuhisa JG, Reichelt M, Gershenzon J. 1991. Variation of glucosinolate accumulation amoung different organs and development stages of Arabidopsis thaliana. Phytochemistry 62: 471-481.

Broz AK, Broeckling CD, De-la-Peña C, Lewis MR, Greene E, Callaway RM, Sumner LW, Vivanco JM. 2010. Plant neighbor identity influences plant biochemistry and physiology related to defense. BMC Plant Biology 10: 1-14.

van Brussel A a N, Tak T, Boot KJM, Kijne JW. 2002. Autoregulation of root nodule formation: signals of both symbiotic partners studied in a split- 
root system of Vicia sativa subsp. nigra. Molecular plant-microbe interactions : MPMI 15: 341-349.

Butler GW, Conn EE. 1964. Biosynthesis of the cyanogenic glucosides linamarin and lotaustralin. The Journal of Biological Chemistry 239: 16741679.

Campbell SA, Kessler A. 2013. Plant mating system transitions drive the macroevolution of defense strategies. Proceedings of the National Academy of Sciences of the United States of America 110: 3973-3978.

Casper BB, Jackson RB. 1997. Plant competition underground. Annual Review of Ecology and Systematics 28: 545-570.

Caswell H. 1989. Matrix Population Models. Sunderland, MA: Sinauer Associates, Inc. Publishers.

Cates RG, Rhoades DF. 1977. Patterns in the production of antiherbivore chemical defenses in plant communities. Biochemical Systematics and Ecology 5: 185-193.

Chase JM, Abrams PA, Grover JP, Diehl S, Chesson P, Holt RD, Richards SA, Nisbet RM, Case TJ. 2002. The interaction between predation and competition: a review and synthesis. Ecology Letters 5: 302315. 


\section{Christensen SA, Nemchenko A, Borrego E, Murray I, Sobhy IS, Bosak}

L, Deblasio S, Erb M, Robert CAM, Vaughn KA, et al. 2013. The maize

lipoxygenase, ZmLOX10, mediates green leaf volatile, jasmonate and herbivore-induced plant volatile production for defense against insect attack. The Plant Journal 74: 59-73.

Cipollini D, Heil M. 2010. Costs and benefits of induced resistance to herbivores and pathogens in plants. CAB Reviews: Perspectives in Agriculture, Veterinary Science, Nutrition and Natural Resources 5: 1-25.

Connell JH. 1983. On the prevalence and relative importance of interspecific competition: evidence from field experiments. American Society of Naturalists 122: 661-696.

Cooper JE. 2007. Early interactions between legumes and rhizobia: disclosing complexity in a molecular dialogue. Journal of Applied Microbiology 103: 1355-65.

Cork SJ. 1996. Optimal digestive strategies for arboreal herbivorous mammals in contrasting forest types: why koalas and colobines are different. Australian Journal of Ecology 21: 10-20.

Crooks JA. 2002. Characterizing ecosystem-level consequences of biological invasions: the role of ecosystem engineers. Oikos 97: 153-166. 
D’Alessandro M, Brunner V, von Mérey G, Turlings TCJ. 2009. Strong attraction of the parasitoid cotesia marginiventris towards minor volatile compounds of maize. Journal of Chemical Ecology 35: 999-1008.

D'Alessandro M, Held M, Triponez Y, Turlings TCJ. 2006. The role of indole and other shikimic acid derived maize volatiles in the attraction of two parasitic wasps. Journal of Chemical Ecology 32: 2733-2748.

Dantas VDL, Batalha MA. 2012. Can antiherbivory resistance explain the abundance of woody species in a Neotropical savanna? Botany 90: 93-99.

Dean JM, Mescher MC, De Moraes CM. 2009. Plant-rhizobia mutualism influences aphid abundance on soybean. Plant and Soil 323: 187-196.

Dean JM, Mescher MC, De Moraes CM. 2014. Plant dependence on rhizobia for nitrogen influences induced plant defenses and herbivore performance. International Journal of Molecular Sciences 15: 1466-1480.

Doust JL, Eaton GW. 1982. Demographic aspects of flower and fruit production in bean plants, Phaseolus vulgaris L. American Journal of Botany 69: $1156-1164$.

Dover BA, Noblet R, Moore RF, Culbertson D. 1988. An improved artificial diet for Mexican bean beetles based on host preference. Journal of Agrigultural Entomology 5: 79-86. 
Ehrlich PR, Raven PH. 1964. Butterflies and plants: a study in coevolution. Evolution 18: 586-608.

Eisner T, Eisner M, Hoebeke ER. 1998. When defense backfires: detrimental effect of a plant's protective trichomes on an insect beneficial to the plant. Proceedings of the National Academy of Sciences of the United States of America 95: 4410-4414.

Van der Ent S, Van Wees SCM, Pieterse CMJ. 2009. Jasmonate signaling in plant interactions with resistance-inducing beneficial microbes. Phytochemistry 70: 1581-1588.

\section{Erb M, Veyrat N, Robert CAM, Xu H, Frey M, Ton J, Turlings TCJ.}

2015. Indole is an essential herbivore-induced volatile priming signal in maize. Nature Communications 6: 6273.

Faeth SH. 1985. Quantitative defense theory and patterns of feeding by oak insects. Oecologia 68: 34-40.

Fenner M, Thompson K. 2005. The Ecology of Seeds. Cambridge: Cambridge University Press.

Flanders R V. 1984. Comparisons of bean varieties currently being used to culture the Mexican bean beetle (Coleoptera: Coccinellidae). Environmental Entomology 13: 995-999. 
Fontana A, Reichelt M, Hempel S, Gershenzon J, Unsicker SB. 2009.

The effects of arbuscular mycorrhizal fungi on direct and indirect defense metabolites of Plantago lanceolata L. Journal of Chemical Ecology 35: 833843.

Frehner M, Conn EE. 1987. The linamarin beta-glucosidase in Costa Rican wild lima beans (Phaseolus lunatus L.) is apoplastic. Plant Physiology 84: $1296-300$.

Ganzhorn JU. 1992. Leaf chemistry and the biomass of folivorous primates in tropical forests. Test of a hypothesis. Oecologia 91: 540-547.

Gao X, Lu X, Wu M, Zhang H, Pan R, Tian J, Li S, Liao H. 2012. Coinoculation with rhizobia and AMF inhibited soybean red crown rot: From field study to plant defense-related gene expression analysis. PLoS ONE 7: $1-10$.

García MB, Ehrlén J. 2002. Reproductive effort and herbivory timing in a perennial herb: fitness components at the individual and population levels. American Journal of Botany 89: 1295-1302.

Géneau CE, Wäckers FL, Luka H, Balmer O. 2013. Effects of extrafloral and floral nectar of Centaurea cyanus on the parasitoid wasp Microplitis mediator: olfactory attractiveness and parasitization rates. Biological Control 
66: $16-20$.

Gleadow RM, Foley WJ, Woodrow IE. 1998. Enhanced CO2 alters the relationship between photosynthesis and defence in cyanogenic Eucalyptus cladocalyx F. Muell. Plant, Cell and Environment 21: 12-22.

Gleadow RM, Møller BL. 2014a. Cyanogenic glycosides: synthesis, physiology, and phenotypic plasticity. Annual Review of Plant Biology 65: $155-185$.

Gleadow RM, Møller BL. 2014b. Cyanogenic Glycosides: Synthesis, Physiology, and Phenotypic Plasticity. Annual Review of Plant Biology 65: $155-185$.

Gleadow RM, Woodrow IE. 2000. Temporal and spatial variation in cyanogenic glycosides in Eucalyptus cladocalyx. Tree Physiology 20: 591-598.

Godschalx AL, Schädler M, Trisel JA, Balkan MA, Ballhorn DJ. 2015a. Ants are less attracted to the extrafloral nectar of plants with symbiotic, nitrogen-fixing rhizobia. Ecology 96: 348-354.

Godschalx AL, Schädler M, Trisel JA, Balkan MA, Ballhorn DJ. 2015b. Ants are less attracted to the extrafloral nectar of plants with symbiotic, nitrogen-fixing rhizobia. Ecology 96: 348-354. 
Godschalx AL, Stady L, Watzig B, Ballhorn DJ. 2016. Is protection against florivory consistent with the optimal defense hypothesis? BMC plant biology 16: 32 .

González-Teuber M, Eilmus S, Muck A, Svatos A, Heil M. 2009.

Pathogenesis-related proteins protect extrafloral nectar from microbial infestation. Plant Journal 58: 464-473.

González-Teuber M, Heil M. 2009. Nectar chemistry is tailored for both attraction of mutualists and protection from exploiters. Plant Signaling \& Behavior 4: 809-813.

González-Teuber M, Silva Bueno JC, Heil M, Boland W. 2012.

Increased host investment in extrafloral nectar (EFN) improves the efficiency of a mutualistic defensive service. PloS One 7: 1-9.

Good AP, Gauthier M-PL, Vannette RL, Fukami T. 2014. Honey bees avoid nectar colonized by three bacterial species, but not by a yeast species, isolated from the bee gut. PloS one 9: 1-8.

Goodger JQD, Ades PK, Woodrow IE. 2004. Cyanogenesis in Eucalyptus polyanthemos seedlings: heritability, ontogeny and effect of soil nitrogen. Tree physiology 24: 681-688.

Gori DF. 1989. Floral color change in Lupinus argenteus (Fabaceae): Why 200 
should plants advertise the location of unrewarding flowers to pollinators?

Evolution 43: 870-881.

Gouinguene SP, Turlings TCJ. 2002. The effects of abiotic factors on induced volatile emissions in corn plants. Plant Physiology 129: 1296-1307.

Goulet H, Huber JT (Eds.). 1993. Hymenoptera of the world: an identification guide to families. Ottowa, Canada: Canada Communication Group Publishing.

Grace JB. 2006. Structural Equation Modeling and Natural Systems. Cambridge, UK: Cambridge University Press.

\section{Grasso DA, Pandolfi C, Bazihizina N, Nocentini D, Nepi M, Mancuso}

S. 2015. Extrafloral-nectar-based partner manipulation in plant-ant relationships. AoB PLANTS 7: 1-15.

Haag JJ, Coupe MD, Cahill JFJ. 2004. Antagonistic interactions between competition and insect herbivory on plant growth. Journal of Ecology 92: $156-167$.

Hahlbrock K, Conn EE. 1970. The biosynthesis of cyanogenic glycosides in higher plants. The Journal of Biological Chemistry 245: 917-922.

Hanson P, Gauld ID (Eds.). 1995. The Hymenoptera of Costa Rica. Oxford 
University Press.

Harris D, Pacovsky R, Paul E. 1985. Carbon economy of soybeanRhizobium-Glomus associations. New Phytologist 101: 427-440.

Hayden KJ, Parker IM. 2002. Plasticity in cyanogenesis of Trifolium repens L.: inducibility, fitness costs and variable expression. Evolutionary Ecology Research 4: 155-168.

Heath JJ, Kessler A, Woebbe E, Cipollini D, Stireman JO. 2014.

Exploring plant defense theory in tall goldenrod, Solidago altissima. New Phytologist 202: 1357-1370.

Hedges L V., Gurevitch J, Curtis PS. 1999. The meta-analysis of response ratios in experimental ecology. Ecology 80: 1150-1156.

Heil M. 2008. Indirect defence via tritrophic interactions. New Phytologist 178: $41-61$.

Heil M. 2011. Nectar: generation, regulation and ecological functions. Trends in Plant Science 16: 191-200.

Heil M. 2015. Extrafloral nectar at the plant-insect interface: a spotlight on chemical ecology, phenotypic plasticity, and food webs. Annual Review of Entomology 60: 213-232. 
Heil M, Koch T, Hilpert A, Fiala B, Boland W, Linsenmair K. 2001.

Extrafloral nectar production of the ant-associated plant, Macaranga tanarius, is an induced, indirect, defensive response elicited by jasmonic acid. Proceedings of the National Academy of Sciences of the United States of America 98: 1083-1088.

\section{Hempel S, Stein C, Unsicker SB, Renker C, Auge H, Weisser WW.} 2009. Specific bottom-up effects of arbuscular mycorrhizal fungi across a plant-herbivore-parasitoid system. Oecologia 160: 267-277.

Hermann SL, Thaler JS. 2014. Prey perception of predation risk: volatile chemical cues mediate non-consumptive effects of a predator on a herbivorous insect. Oecologia 176: 669-76.

Herms DA, Mattson WJ. 1992. The dilemma of plants: to grow or defend. The Quarterly Review of Biology 67: 283-335.

\section{HilleRisLambers J, Adler PB, Harpole WS, Levine JM, Mayfield MM.} 2012. Rethinking Community Assembly through the Lens of Coexistence Theory. Annual Review of Ecology, Evolution, and Systematics 43: 227-248.

Hoballah FEM, Turlings TCJ. 2001. Experimental evidence that plants under caterpillar attack may benefit from attracting parasitoids caterpillar attack may benefit from attracting parasitoids. Evolutionary Ecology 
Research 3: 553-565.

Hoffmeister M, Junker RR. 2017. Herbivory-induced changes in the olfactory and visual display of flowers and extrafloral nectaries affect pollinator behavior. Evolutionary Ecology 31: 269-284.

Holman L, Dreier S, D’Ettorre P. 2010. Selfish strategies and honest signalling: reproductive conflicts in ant queen associations. Proceedings of the Royal Society of Biological Sciences 277: 2007-2015.

Howell AD, Alarcón R. 2007. Osmia bees (Hymenoptera: Megachilidae) can detect nectar-rewarding flowers using olfactory cues. Animal Behaviour 74: $199-205$.

Inui Y, Itioka T. 2007. Species-specific leaf volatile compounds of obligate macaranga myrmecophytes and host-specific aggressiveness of symbiotic crematogaster ants. Journal of Chemical Ecology 33: 2054-2063.

Izaguirre MM, Mazza CA, Astigueta MS, Ciarla AM, Ballaré CL. 2013. No time for candy: passionfruit (Passiflora edulis) plants down-regulate damage-induced extra floral nectar production in response to light signals of competition. Oecologia 173: 213-221.

Jia Y, Gray VM. 2008. Growth yield of Vicia faba L in response to microbial symbiotic associations. South African Journal of Botany 74: 25-32. 
Johnson MTJ. 2008. Bottom-up effects of plant genotype on aphids, ants, and predators. Ecology 89: 145-54.

Jones EI, Afkhami ME, Akçay E, Bronstein JL, Bshary R, Frederickson ME, Heath KD, Hoeksema JD, Ness JH, Pankey MS, et al. 2015. Cheaters must prosper: reconciling theoretical and empirical perspectives on cheating in mutualism. Ecology Letters 18: 1270-1284.

Jones PR, Møller BL, Høj PB. 1999. The UDP-glucose: phydroxymandelonitrile-O-glucosyltransferase that catalyzes the last step in synthesis of the cyanogenic glucoside dhurrin in Sorghum bicolor. Journal of Biological Chemistry 274: 35483-35491.

Junker RR, Blüthgen N. 2008. Floral scents repel potentially nectarthieving ants. Evolutionary Ecology Research 10: 295-308.

Kakes P. 1990. Properties and functions of the cyanogenic system in higher plants. Euphytica 48: 25-43.

Kaplan I, Dively GP, Denno RF. 2009. The costs of anti-herbivore defense traits in agricultural crop plants: a case study involving leafhoppers and trichomes. Ecological applications : a publication of the Ecological Society of America 19: 864-72.

Kaschuk G, Kuyper TW, Leffelaar PA, Hungria M, Giller KE. 2009. Are 205 
the rates of photosynthesis stimulated by the carbon sink strength of rhizobial and arbuscular mycorrhizal symbioses? Soil Biology and Biochemistry 41: 1233-1244.

Kautz S, Trisel J a., Ballhorn DJ. 2014. Jasmonic acid enhances plant cyanogenesis and resistance to herbivory in lima bean. Journal of Chemical Ecology 40: 1186-1196.

Kempel A, Brandl R, Schädler M. 2009a. Symbiotic soil microorganisms as players in aboveground plant-herbivore interactions - the role of rhizobia. Oikos 118: 634-640.

Kempel A, Brandl R, Schädler M. 2009b. Symbiotic soil microorganisms as players in aboveground plant-herbivore interactions - the role of rhizobia. Oikos 118: 634-640.

Kempel A, Schädler M, Chrobock T, Fischer M, van Kleunen M. 2011. Tradeoffs associated with constitutive and induced plant resistance against herbivory. Proceedings of the National Academy of Sciences of the United States of America 108: 5685-5689.

Kessler D, Baldwin IT. 2007. Making sense of nectar scents: The effects of nectar secondary metabolites on floral visitors of Nicotiana attenuata. Plant Journal 49: 840-854. 
Kessler A, Halitschke R. 2009a. Testing the potential for conflicting selection on floral chemical traits by pollinators and herbivores: predictions and case study. Functional Ecology 23: 901-912.

Kessler A, Halitschke R. 2009b. Testing the potential for conflicting selection on floral chemical traits by pollinators and herbivores: Predictions and case study. Functional Ecology 23: 901-912.

Kiers ET, Rousseau RA, Denison RF. 2006. Measured sanctions: legume hosts detect quantitative variation in rhizobium cooperation and punish accordingly. Evolutionary Ecology Research: 1077-1086.

Kiers ET, Rousseau RA, West SA, Denison RF. 2003. Host sanctions and the legume-rhizobium mutualism. Nature 425: 78-81.

Kost C, Heil M. 2005. Increased availability of extrafloral nectar reduces herbivory in Lima bean plants (Phaseolus lunatus, Fabaceae). Basic and Applied Ecology 6: 237-248.

Kost C, Heil M. 2006. Herbivore-induced plant volatiles induce an indirect defence in neighbouring plants. Journal of Ecology 94: 619-628.

Kost C, Heil M. 2008. The defensive role of volatile emission and extrafloral nectar secretion for lima bean in nature. Journal of Chemical Ecology 34: 113. 


\section{Kouchi H, Imaizumi-Anraku H, Hayashi M, Hakoyama T, Nakagawa}

T, Umehara Y, Suganuma N, Kawaguchi M. 2010. How many peas in a pod? Legume genes responsible for mutualistic symbioses underground. Plant and Cell Physiology 51: 1381-1397.

Kranthi S, Kranthi KR, Wanjari RR. 2003. Influence of semilooper damage on cotton host-plant resistance to Helicoverpa armigera (Hub). Plant Science 164: 157-163.

Kursar T., Coley P. 2003. Convergence in defense syndromes of young leaves in tropical rainforests. Biochemical Systematics and Ecology 31: 929949.

Laird R a, Addicott JF. 2007. Arbuscular mycorrhizal fungi reduce the construction of extrafloral nectaries in Vicia faba. Oecologia 152: 541-551.

LaPidus JB, Cleary RW, Davidson RH, Fisk FW, Augustine MG. 1963. Chemical factors influencing host selection by Mexican bean beetle Epilachna varivestis Muls. Agricultural and Food Chemistry 11: 462-463.

Letourneau DK, Jedlicka J a., Bothwell SG, Moreno CR. 2009. Effects of Natural Enemy Biodiversity on the Suppression of Arthropod Herbivores in Terrestrial Ecosystems. Annual Review of Ecology, Evolution, and Systematics 40: 573-592. 


\section{Li T, Holopainen JK, Kokko H, Tervahauta AI, Blande JD. 2012.}

Herbivore-induced aspen volatiles temporally regulate two different indirect defences in neighbouring plants. Functional Ecology 26: 1176-1185.

Lieberei R. 1988. Relationship of Cyanogenic Capacity ( $\mathrm{HCN}-\mathrm{c}$ ) of the Rubber Tree Hevea brasUiensis to Susceptibility to Microcydus ulei, the Agent Causing South American Leaf Blight. 67.

Lieberei R, Biehl B, Giesemann A, Junqueira NT. 1989. Cyanogenesis inhibits active defense reactions in plants. Plant Physiology 90: 33-36.

de Lima Mendonça A, Silva CE, de Mesquita FLT, da Silva Campos R, Do Nascimento RR, de Azevedo Ximenes ECP, Sant’ Ana AEG. 2009. Antimicrobial activities of components of the glandular secretions of leaf cutting ants of the genus Atta. Antonie van Leeuwenhoek, International Journal of General and Molecular Microbiology 95: 295-303.

Limburg DD, Rosenheim JA. 2001. Extrafloral nectar consumption and its influence on survival and development of an omnivorous predator, larval Chrysoperla plorabunda (Neuroptera: Chrysopidae). Environmental Entomology 30: 595-604.

Liu Y, Wang WL, Guo GX, Ji XL. 2009. Volatile emission in wheat and parasitism by Aphidius avenae after exogenous application of salivary 
enzymes of Sitobion avenae. Entomologia Experimentalis et Applicata 130: $215-221$.

Lodwig E, Hosie A, Bourdes A, Findlay K, Allaway D, Karunakaran R, Downie J, Poole P. 2003. Amino-acid cycling drives nitrogen fixation in the legume-Rhizobium symbiosis. Nature $\mathbf{4 2 2}$.

Lou Y, Baldwin IT. 2004. Nitrogen supply influences herbivore-induced direct and indirect defenses and transcriptional responses in Nicotiana attenuata. Plant Physiology 135: 496-506.

Luo S, Michaud JP, Li J, Liu X, Zhang Q. 2013. Odor learning in Microplitis mediator (Hymenoptera: Braconidae) is mediated by sugar type and physiological state. Biological Control 65: 207-211.

Marak HB, Biere a., Van Damme JMM. 2000. Direct and correlated responses to selection on iridoid glycosides in Plantago lanceolata L. Journal of Evolutionary Biology 13: 985-996.

Marak HB, Biere A, Van Damme JMM. 2003. Fitness costs of chemical defense in Plantago lanceolata L.: effects of nutrient and competition stress. Evolution; International Journal of Organic Evolution 57: 2519-2530.

Massad TJ, Dyer LA, Vega CG. 2012. Costs of defense and a test of the carbon-nutrient balance and growth-differentiation balance hypotheses for 
two co-occurring classes of plant defense. PloS one 7: e47554.

Mayer VE, Frederickson ME, Mckey D, Blatrix R. 2014. Current issues in the evolutionary ecology of ant - plant symbioses. New Phytologist.

McCall AC, Irwin RE. 2006. Florivory: The intersection of pollination and herbivory. Ecology Letters 9: 1351-1365.

McKey D. 1974. Adaptive patterns in alkaloid physiology. The American Naturalist 108: 305-320.

McLain DK. 1983. Ants, extrafloral nectaries and herbivory on the passion vine, Passiflora incarnata. American Midland Naturalist 110: 433-439.

McNaughton SJ. 1983. Compensatory plant growth as a response to herbivory. Oikos 40.

Moles AT, Peco B, Wallis IR, Foley WJ, Poore AGB, Seabloom EW, Vesk P a, Bisigato AJ, Cella-Pizarro L, Clark CJ, et al. 2013.

Correlations between physical and chemical defences in plants: tradeoffs, syndromes, or just many different ways to skin a herbivorous cat? The New phytologist: 252-263.

Mondor EB, Tremblay MN, Messing RH. 2006. Extrafloral nectary phenotypic plasticity is damage- and resource-dependent in Vicia faba. 
Biology letters 2: 583-585.

De Moraes CM, Lewis WJ, Paré PW, Alborn HT, Tumlonson JH, Pare PW. 1998. Herbivore-infested plants selectively attract parasitoids. Nature 393: $570-573$.

Moser JC, Brownlee R, Silverstein R. 1968. Alarm pheromones of the ant Atta texana. Journal of Insect Physiology 14: 529-530.

Mothershead K, Marquis RJ. 2000. Fitness impacts of herbivory through indirect effects on plant-pollinator interactions in Oenothera macrocarpa. Ecology 81: 30-40.

De Moura RL, Foster KW. 1986. Effects of cultivar and flower removal treatments on the temporal distribution of reproductive structures in bean. Crop Science 26: 362-367.

Mueller MJ, Brodschelm W, Spannagl E, Zenk MH. 1993. Signaling in the elicitation process is mediated through the octadecanoid pathway leading to jasmonic acid. Proceedings of the National Academy of Sciences of the United States of America 90: 7490-7494.

Nei M, Li W-H. 1979. Mathematical model for studying genetic variation in terms of restriction endonucleases. Proceedings of the National Academy of Sciences of the United States of America 76: 5269-5273. 
Nepi M, Guarnieri M, Pacini E. 2001. Nectar secretion, reabsorption, and sugar composition in male and female flowers of Cucurbita pepo.

International Journal of Plant Sciences 162: 353-358.

Ness JH. 2006. A mutualism's indirect costs: the most aggressive plant bodyguards also deter pollinators. Oikos 113: 506-514.

Ness JH, Morris WF, Bronstein JL. 2009. For ant-protected plants, the best defense is a hungry offense. Ecology 90: 2823-2831.

Nienhuis J, Tivang J, Skroch P, dos Santos JB. 1995. Genetic relationships among cultivars and landraces of lima bean (Phaseolus lunatus L .) as measured by RAPD markers. Journal of American Society of Horticultural Sciences 120: 300-306.

Noitsakis B, Jacquard P. 1992. Competition between cyanogenic and acyanogenic morphs of Trifolium repens. Theoretical and Applied Genetics 83: $443-450$.

Nomura M, Hatada A, Itioka T. 2011. Correlation between the leaf turnover rate and anti-herbivore defence strategy (balance between ant and non-ant defences) amongst ten species of Macaranga (Euphorbiaceae). Plant Ecology 212: 143-155.

Nunes-Nesi A, Fernie AR, Stitt M. 2010. Metabolic and signaling aspects 213 
underpinning the regulation of plant carbon nitrogen interactions. Molecular Plant 3: 973-996.

Nuringtyas TR, Verpoorte R, Klinkhamer PGL, van Oers MM, Leiss K a. 2014. Toxicity of Pyrrolizidine Alkaloids to Spodoptera exigua Using Insect Cell Lines and Injection Bioassays. Journal of Chemical Ecology.

O'Dowd DJ. 1979. Foliar nectar production and ant activity on a neotropical tree, Ochroma pyramidale. Oecologia 43: 233-248.

O’Hara RB, Kotze DJ. 2010. Do not log-transform count data. Methods in Ecology and Evolution 1: 118-122.

Ohnmeiss TE, Baldwin IT. 2000. Optimal defense theory predicts the ontogeny of an induced nicotine defense. Ecology 81: 1765-1783.

Oka-Kira E, Kawaguchi M. 2006. Long-distance signaling to control root nodule number. Current Opinion in Plant Biology 9: 496-502.

Paliy O, Shankar V. 2016. Application of multivariate statistical techniques in microbial ecology. Molecular Ecology 25: 1032-1057.

Pangesti N, Weldegergis BT, Dicke M, Pineda A. 2015a. Rhizobacterial colonization of roots modulates plant volatile emission and enhances the attraction of a parasitoid wasp to host - infested plants. Oecologia: 1169- 
1180.

Pangesti N, Weldegergis BT, Dicke M, Pineda A. 2015b. Rhizobacterial colonization of roots modulates plant volatile emission and enhances the attraction of a parasitoid wasp to host - infested plants. Oecologia: 11691180.

Pangesti N, Weldegergis BT, Langendorf B, van Loon JJ a., Dicke M, Pineda A. 2015c. Rhizobacterial colonization of roots modulates plant volatile emission and enhances the attraction of a parasitoid wasp to hostinfested plants. Oecologia: 1169-1180.

Patt JM, Pfannenstiel RS. 2008. Odor-based recognition of nectar in cursorial spiders. Entomologia Experimentalis et Applicata 127: 64-71.

Penet L, Collin CL, Ashman TL. 2009. Florivory increases selfing: An experimental study in the wild strawberry, Fragaria virginiana. Plant Biology 11: 38-45.

Penmetsa RV, Cook D. 1997. A legume ethylene-insensitive mutant hyperinfected by its rhizobial symbiont. Science 275: 527-530.

Peoples MB, Pate JS, Atkins CA, Bergersen FJ. 1986. Nitrogen nutrition and xylem sap composition of peanut (Arachis hypogaea L. cv Virginia Bunch). Plant Physiology 82: 946-951. 


\section{Pineda A, Soler R, Weldegergis BT, Shimwela MM, van Loon JJA,}

Dicke M. 2013. Non-pathogenic rhizobacteria interfere with the attraction of parasitoids to aphid-induced plant volatiles via jasmonic acid signalling. Plant, Cell and Environment 36: 393-404.

Pineda A, Zheng SJ, van Loon JJA, Dicke M. 2012. Rhizobacteria modify plant-aphid interactions: A case of induced systemic susceptibility. Plant Biology 14: 83-90.

Pineda A, Zheng S-J, van Loon JJ a, Pieterse CMJ, Dicke M. 2010. Helping plants to deal with insects: the role of beneficial soil-borne microbes. Trends in Plant Science 15: 507-514.

Poulton JE. 1990. Cyanogenesis in plants. Plant Physiology 94: 401-405.

\section{Prell J, White JP, Bourdes A, Bunnewell S, Bongaerts RJ, Poole PS.}

2009. Legumes regulate Rhizobium bacteroid development and persistence by the supply of branched-chain amino acids. Proceedings of the National Academy of Sciences of the United States of America 106: 12477-12482.

Pringle EG. 2015a. Integrating plant carbon dynamics with mutualism ecology. New Phytologist.

Pringle EG. 2015b. Integrating plant carbon dynamics with mutualism ecology. New Phytologist 210: 71-75. 
Pulice CE, Packer AA. 2008. Simulated herbivory induces extrafloral nectary production in Prunus avium. Functional Ecology 22: 801-807.

Radhika V, Kost C, Bartram S, Heil M, Boland W. 2008. Testing the optimal defence hypothesis for two indirect defences: extrafloral nectar and volatile organic compounds. Planta 228: 449-457.

Radhika V, Kost C, Boland W, Heil M. 2010a. The role of jasmonates in floral nectar secretion. PloS one 5: e9265.

Radhika V, Kost C, Mithöfer A, Boland W. 2010b. Regulation of extrafloral nectar secretion by jasmonates in lima bean is light dependent. Proceedings of the National Academy of Sciences of the United States of America 107: 17228-33.

Raguso RA. 2004. Why are some floral nectars scented? Ecology 85: 14861494.

Rask L, Andréasson E, Ekbom B, Eriksson S, Pontoppidan B, Meijer J. 2000. Myrosinase: gene family evolution and herbivore defense in Brassicaceae. Plant molecular biology 42: 93-113.

Rasmann S, Bennett A, Biere A, Karley A, Guerrieri E. 2017. Root symbionts: Powerful drivers of plant above- and belowground indirect defences. Insect Science. 
Rasmann S, Köllner TG, Degenhardt J, Hiltpold I, Toepfer S, Kuhlmann U, Gershenzon J, Turlings TC. 2005. Recruitment of entomopathogenic nematodes by insect-damaged maize roots. Nature 434: $732-737$.

\section{Read J, Sanson GD, Caldwell E, Clissold FJ, Chatain A, Peeters P,} Lamont BB, De Garine-Wichatitsky M, Jaffré T, Kerr S. 2009.

Correlations between leaf toughness and phenolics among species in contrasting environments of Australia and New Caledonia. Annals of Botany 103: $757-67$.

Rees M. 1995. Community structure in sand dune annuals: is seed weight a key quantity? Oecologia 83: 857-863.

Rees M, Brown VK. 1992. Interactions between invertebrate herbivores and plant competition. Journal of Ecology 80: 353-360.

Röse USR, Lewis J, Tumlinson JH. 2006. Extrafloral nectar from cotton (Gossypium hirsutum) as a food source for parasitic wasps. Functional Ecology 20: 67-74.

Rudgers JA, Gardener MC. 2004. Extrafloral nectar as a resource mediating multispecies interactions. Ecology 85: 1495-1502.

Schädler M, Brandl R, Haase J. 2007. Antagonistic Interactions between 
Plant Competition and Insect Herbivory. Ecology 88: 1490-1498.

Schowalter TD. 1981. Insect herbivore relationship to the state of the host plant: biotic regulation of ecosystem nutrient cycling through ecological succession. Oikos 37: 126-130.

Seigler D, Price PW. 1976. Secondary compounds in plants: primary functions. The American Naturalist 110: 101-105.

Siemens DH, Garner SH, Mitchell-Olds T, Callaway RM. 2002. Cost of defense in the context of plant competition: Brassica rapa may grow and defend. Ecology 83: 505-517.

Simms EL, Taylor DL. 2002. Partner choice in nitrogen-fixation mutualisms of legumes and rhizobia. Integrative and comparative biology 42: $369-380$.

Simon J, Gleadow RM, Woodrow IE. 2010. Allocation of nitrogen to chemical defence and plant functional traits is constrained by soil N. Tree Physiology 30: 1111-1117.

Smith JM, Arteca RN, McMahon Smith J, Arteca RN. 2000. Molecular control of ethylene production by cyanide in Arabidopsis thaliana. Physiologia Plantarum 109: 180-187. 
Southwick EE. 1984. Photosynthate allocation to floral nectar: a neglected energy investment. Ecology 65: 1775-1779.

Stamp N. 2003. Out of the quagmire of plant defense hypotheses. The Quarterly Review of Biology 78: 23-55.

Stanton ML. 2003. Interacting guilds: moving beyond the pairwise perspective on mutualisms. The American naturalist 162: S10-23.

Strauss SY. 1997. Floral characters link herbivores, pollinators, and plant fitness. Ecology 78: 1640-1645.

Strauss SY, Whittall JB. 2006. Non-pollinator agents of selection on floral traits. Ecology and Evolution of Flowers.120-139.

Summers M, Mondor E. 2011. Rhizobium alters inducible defenses in broad bean, Vicia faba. Open Journal of Ecology 1: 57-62.

Sun J, Cardoza V, Mitchell DM, Bright L, Oldroyd G, Harris JM. 2006.

Crosstalk between jasmonic acid, ethylene and Nod factor signaling allows integration of diverse inputs for regulation of nodulation. The Plant Journal 46: $961-970$.

Tan X-L, Liu T-X. 2014. Aphid-induced plant volatiles affect the attractiveness of tomato plants to Bemisia tabaci and associated natural 
enemies. Entomologia Experimentalis et Applicata: n/a-n/a.

Thaler JS. 1999. Jasmonate-inducible plant defences cause increased parasitism of herbivores. Nature 399: 17-19.

Thamer S, Schädler M, Bonte D, Ballhorn DJ. 2011. Dual benefit from a belowground symbiosis: nitrogen fixing rhizobia promote growth and defense against a specialist herbivore in a cyanogenic plant. Plant and Soil 341: 209219.

Tigreros N, Norris RH, Wang EH, Thaler JS. 2017. Maternally induced intraclutch cannibalism: an adaptive response to predation risk? Ecology Letters: 487-494.

\section{Tsikou D, Kalloniati C, Fotelli MN, Nikolopoulos D, Katinakis P,} Udvardi MK, Rennenberg H, Flemetakis E. 2013. Cessation of photosynthesis in Lotus japonicus leaves leads to reprogramming of nodule metabolism. Journal of Experimental Botany 64: 1317-1332.

Turley NE, Godfrey RM, Johnson MJT. 2013. Evolution of mixed strategies of plant defense against herbivores. New Phytologist 197: 359-361.

Turlings TCJ, Tumlinson JH, Lewis WJ. 1990. Exploitation of herbivoreinduced plant odors by host-seeking parasitic wasps. Science 250: 1251-1253. 
Turlings TCJ, Wäckers F. 2004. Recruitment of predators and parasitoids by herbivore-injured plants. In: Cárde R,, In: JG M, eds. Advances in Insect Chemical Ecology. West Nyack, NY, 21-75.

Turlings TCJ, Wäckers FL, Vet LEM, Lewis WJ, Tumlinson JH. 1993a. Learning of host-finding cues by Hymenopterous parasitoids. Insect Learning: Ecological and Evolutionary Perspectives 3: 51-78.

Turlings TCJ, Wäckers FL, Vet LEM, Lewis J, Tumlinson JH. 1993b.

Learning of host-finding cues by hymenopterous parasitoids. Insect Learning: Ecological and Evolutionary Perspectives 3: 51-78.

Vannette RL, Fukami T. 2016. Nectar microbes can reduce secondary metabolites in nectar and alter effects on nectar consumption by pollinators. Ecology 97: 1410-1420.

Vannette RL, Gauthier M-PL, Fukami T. 2013. Nectar bacteria, but not yeast, weaken a plant-pollinator mutualism. Proceedings of The Royal Society 280: $1-7$.

de Vega C, Herrera CM. 2013. Microorganisms transported by ants induce changes in floral nectar composition of an ant-pollinated plant. American Journal of Botany 100: 792-800.

Verchot L V., Moutinho PR, Davidson EA. 2003. Leaf-cutting ant (Atta 222 
Sexdens) and nutrient cycling: deep soil inorganic nitrogen stocks, mineralization, and nitrification in Eastern Amazonia. Soil Biology and Biochemistry 35: 1219-1222.

Vessey JK, Pawlowski K, Bergman B. 2004. Root-based N2 -fixing symbioses: Legumes, actinorhizal plants, Parasponia sp. and cycads. Plant and Soil 266: 205-230.

Vetter J. 2000. Plant cyanogenic glycosides. Toxicon: official journal of the International Society on Toxinology 38: 11-36.

Veyrat N, Robert CAM, Turlings TCJ, Erb M. 2016. Herbivore intoxication as a potential primary function of an inducible volatile plant signal. Journal of Ecology 104: 591-600.

Viola D V, Mordecai EA, Jaramillo AG, Sistla SA, Albertson LK, Gosnell JS, Cardinale BJ, Levine JM. 2010. Competition-defense tradeoffs and the maintenance of plant diversity. Proceedings of the National Academy of Sciences of the United States of America 107: 17217-22.

Vitousek PM, Hättenschwiler S, Olander L, Allison S. 2002. Nitrogen and nature. Ambio 31: 97-101.

Wäckers FL, Bonifay C. 2004. How to be sweet? Extrafloral nectar allocation by Gossypium hirsutum fits optimal defense theory predictions. 
Ecology 85: 1512-1518.

Wagner D. 1997. The influence of ant nests on Acacia seed production, herbivory and soil nutrients. Journal of Ecology 85: 83-93.

Wanjiku C, Khamis FM, Teal PEA, Torto B. 2014. Plant volatiles influence the African weaver ant-cashew tree mutualism. Journal of Chemical Ecology 40: 1167-1175.

Weber MG, Agrawal A a. 2014. Defense mutualisms enhance plant diversification. Proceedings of the National Academy of Sciences of the United States of America 111: 16442-7.

Weber MG, Keeler KH. 2013. The phylogenetic distribution of extrafloral nectaries in plants. Annals of Botany 111: 1251-1261.

Wei J, Van Loon JJA, Rieta G, Menzel TR, Li N, Kang L, Dicke M. 2014a. Reciprocal crosstalk between jasmonate and salicylate defencesignalling pathways modulates plant volatile emission and herbivore hostselection behaviour. Journal of Experimental Botany 65: 3289-3298.

Wei J, Loon JJA Van, Gols R, Menzel TR, Li N, Kang L, Dicke M. 2014b. Reciprocal crosstalk between jasmonate and salicylate defencesignalling pathways modulates plant volatile emission and herbivore hostselection behaviour. 65: 3289-3298. 
Werner GDA, Strassmann JE, Ivens ABF, Engelmoer DJP, Verbruggen E, Queller DC, Noë R, Johnson NC, Hammerstein P, Kiers ET. 2014. Evolution of microbial markets. Proceedings of the National Academy of Sciences of the United States of America 111: 1237-1244.

Willmer PG, Nuttman C V., Raine NE, Stone GN, Pattrick JG, Henson K, Stillman P, McIlroy L, Potts SG, Knudsen JT. 2009. Floral volatiles controlling ant behaviour. Functional Ecology 23: 888-900.

Wilson SD, Tilman D. 1991. Component of plant competition along an experimental gradient of nitrogen availability. Ecology 72: 1050-1065.

Winter TR, Rostás M. 2010. Nitrogen deficiency affects bottom-up cascade without disrupting indirect plant defense. Journal of Chemical Ecology 36: $642-651$.

Wise MJ, Abrahamson WG. 2007. Effects of resource availability on tolerance of herbivory: a review and assessment of three opposing models. The American Naturalist 169: 443-454.

Wise MJ, Cummins JJ, De Young C. 2008. Compensation for floral herbivory in Solanum carolinense: identifying mechanisms of tolerance. Evolutionary Ecology 22: 19-37.

Wright GA, Baker DD, Palmer MJ, Stabler D, Mustard JA, Power EF, 225 
Borland AM, Stevenson PC. 2013. Caffeine in floral nectar enhances a pollinator's memory of reward. Science 339: 1202-1204.

Wright DP, Read DJ, Scholes JD. 1998. Mycorrhizal sink strength influences whole plant carbon balance of Trifofium repens L. Plant, Cell and Environment 21: 881-891.

Young B, Wagner D, Doak P, Clausen T. 2010. Within-plant distribution of phenolic glycosides and extrafloral nectaries in trembling aspen (Populus tremuloides; Salicaceae). American Journal of Botany 97: 601-610.

Youngsteadt E, Nojima S, Häberlein C, Schulz S, Schal C. 2008. Seed odor mediates an obligate ant-plant mutualism in Amazonian rainforests. Proceedings of the National Academy of Sciences of the United States of America 105: 4571-4575.

Zangerl AR, Bazzaz FA. 1992. Theory and pattern in plant defense allocation. In: Fritz RS,, In: Simms EL, eds. Plant Resistance to Herbivores and Pathogens: Ecology, Evolution, and Genetics.

Zangerl AR, Rutledge CE. 1996. The probability of attack and patterns of constitutive and induced defense: a test of optimal defense theory. The American Naturalist 147: 599-608.

Zenk MH. 1967. Biochemistry and physiology of secondary plant substances. 
Berichte der Deutschen Botanischen Gesellschaft 80: 573-591. 